University of Louisville

ThinkIR: The University of Louisville's Institutional Repository

Electronic Theses and Dissertations

$12-1977$

\title{
A new procedure for calculating dipolar sources of EEG discharges.
}

Joseph Harvey Rothweiler 1952-

University of Louisville

Follow this and additional works at: https://ir.library.louisville.edu/etd

\section{Recommended Citation}

Rothweiler, Joseph Harvey 1952-, "A new procedure for calculating dipolar sources of EEG discharges." (1977). Electronic Theses and Dissertations. Paper 1237.

https://doi.org/10.18297/etd/1237

This Master's Thesis is brought to you for free and open access by ThinkIR: The University of Louisville's Institutional Repository. It has been accepted for inclusion in Electronic Theses and Dissertations by an authorized administrator of ThinkIR: The University of Louisville's Institutional Repository. This title appears here courtesy of the author, who has retained all other copyrights. For more information, please contact thinkir@louisville.edu. 
A NEW PROCEDURE FOR CALCULATING

DIPÖLAR SOURCES OF EEG DISCHARGES

\author{
By \\ Joseph Harvey Rothweiler \\ ,
}

\begin{abstract}
A Thesis
Submitted to the Faculty of the

University of Louisville

Speed Scientific School

as Partial Fulfillment of the Requirerents

for the Professional Degree
\end{abstract}

MASTER OF ENGINEERING

Department of Electrical Engineering

December 1977 
A New Procedure for Calculating Dipolar Sources of EEG Discharges

Submitted by: Joseph Harvey Rothweiler

A Thesis Approved on

November 22, 1977

by the Following Reading and Examination Committee:

Thesis Director, William H. Pierce

Thomas G. Cleaver

Larry Tyler 
ABSTRACT

A new procedure has been developed for determining the location of the source of certain EEG discharges given the measured surface potentials. The source is modeled as a single current dipole with arbitrary position and orientation, while the head is modeled either as a single homogeneous sphere or as three concentric spheres.

This procedure is developed by extending the matched filter concept of communications theory to functions defined on a spherical surface. In addition, simplifications are developed which reduce the amount of computation required. Simulations have been performed using computer generated data to show the validity of the locating procedure and to investigate its sensitivity to changes in source location.

It is shown that the new procedure has approximately the same accuracy as previously used techniques, and possible computational advantages of the new procedure aro discussed. 
TABLE OF CONTENTS

Page

I. BACKGROUND AND SCOPE OF THESIS

II. THE MATCHED FILTER DETECTION PROCESS 9

III. SIMULATIONS 38

IV. SUMMARY 60

V. RECOMMENDATIONS FOR FUTURE WORK 62

APPENDIX A. SURFACE POTENTIAL EQUATIONS 67

APPENDIX B. COMPUTER PROGRAMS AND OUTPUTS 75

APPEIDIX C. EFFECTS OF NOISE 137

BIBLIOGRAPHY 143

$\begin{array}{ll}\text { VITA } & 146\end{array}$

38
60
62
67
5
37
43
146




\section{LIST OF TABLES}

\section{Page}

TABLE I. RELATIVE SIGNAL ENERGIES PRODUCED BY CURRENT DIPOLES

TABLE A 1. PARAMETERS OF THE THREE SPHERES MODEL 
LIST OF ILLUSTRATIONS

Page

FIGURE 1. The Single Sphere Model of the Humsn Head

FTGURE 2. Surface Potential of a Radial

Dipole on the $Z$ Axis as a Function of $\theta$ for

Different values of $r$

FIGURE 3. Surface Potential of a Tangential

Dipole on the $Z$ Axis as a Function of $\theta$ for

Different Values of $r$

FIGURE 4. Filter Response for Position

Change with Depth Constant

FIGURE 5. Filter Response for Depth Mismatch Between Filter and Dipole

FIGURE 6. Response of a Radial filter for Depth Mismatch with Varying Numbers of

Electrodes

FIGURE 7. Response of a Tangential Filter for Depth Mismatch with Varying Numbers of Electrodes

FIGURE 8. Radial Filter Response for Depth Mismatch with Electrodes on One Hemisphere only

FIGURE 9. Tangential Filter Response for Depth Mismotch with Electrodes on One Hemisphere only

FIGURE A 1. The Three Spheres Model of the Human Head

FIGURE BI. Program to Calculate Theoretical Surface Potentials

FIGURE B2. Theoretical Surface Potentials of a Radial Dipole

FIGURE B3. Theoretical Surface Potentials of a Tongential Dipole 
Page

FIGURE Bly. Program to Test Numerical

Integration for Depth Mismatch

82

FIGURE B5. Integral Values for Depth Mismatch with a Radiel Dipole

83

FIGURE B6. Integral Values for Depth Mismatch with a Tangential Dipole

91

FIGURE B7. Final Program to Evaluate Filter

Response for Depth Mismatch

102

FIGURE B8. Computed Rodial Filter Response

for Depth Mismatch

104

FIGURE B9. Computed Tangential Filter

Response for Depth Mismetch

105

FIGURE BIO. Program to Calculate Filter

Response for General Position Mismatch

107

FIGURE Bll. Simulation Results for General

Position Variation

111

FIGURE B12. Rectangular Integration Metbod

Used for Integration over Theta

FIGURE B13. Simulation Program for the

Discrete Case

117

FIGURE Bl4. Simulation Results for the

Discrete Case Using a Radial Dipole

122

FIGURE B15. Simulation Results for the

Discrete Case Using a Tangential Dipole

125

FIGURE B16. DIvision of a Hemisphere into

Bands of Equal Width

131

FIGURE B17. Division of a Hemisphere into Rings of Equal Ares 
I. BACKGROUND AND SCOPE OF THESIS

A general problem whlch exists in electroencephalography and electrocardiography is to determine the relationship between tho potentials on the surface of a volume conductor and the internal sources. The forward problem, finding the surface potentials given the source distribution, can be solved by applying electromagnetic theory to an electrical model of the biological system under study. ?

A difficulty in the inverse problem, determining the sources given the surface potentials, is the lack of unlqueness. It has been shown that the surface potentials do not provide enough information to completely specify the source distribution. 2 A way of dealing with thls problem is to use an electrical model of the blological system in which the source structure is restricted in such a wa that it is uniquely specified by the surface potentials. Some approximations which have been shown to bo valld for most models of the volume conductor are that the medium is Inear and that capacitive and Inductive effects and propagetion delays are negligible at the frequencies of Interest. ${ }^{3}$ As a result, problems may be treated as if steady state conditions existed at any instent; and the static, rather than time varying, forms of the eloctro- 
magnetic equations mag bo used.

Two models of the head are widely used in studies of the EEG. A simple model is a homogeneous sphere. A more complex model consists of three concentric spheres with different conductivities, representing the scalp, skull, and brain. It has been shown that the three spheres model is quite accurate in most cases. 4,5

It is desirable that the restrictions placed on the source structure be related to the physiological characteristics of the system being studied. In EEG investigations it has been shown that the source of the spike discharges observed in certain forms of epllepsy can be modeled as a single current dipole located in the brain. 6 Studies of artiflcially induced epilepsy is animals have observed the formation of secondary sources of opileptic activity in the brain. 7-10 Whether these socondary sources may 8180 be modeled as current dipoles is not known.

The spproach to tho inverse problem used by Rogers and P1lkingtonll, 12 for olectrocardiographic applications is to model the source as a number of current dipoles which are fixed in position and orientation, but have variable magnitudes. Since the volume conductor la linear, the surface potential is a linear function of the dipole magnitudes. A dipole with random orientation may then be represented by resolving it into three component dipoles. The relationship between the surface potentials at $M$ locations and $N$ dipole magnitudes is described as 
follows. The M-dimensional column vector $\Phi$ conslsts of the observed electrode voltages. The electrode potentiels due to the 1 'th dipole are modeled as $\mathrm{C}_{1} \mathrm{p}_{1}$, where $\mathrm{p}_{1}$ is the dipole magnitude, and the M-dimensional rector $C_{1}$ is computed based on an electrical model of the system under study. The surface potential is then modeled as loq. 7 of ref. 12):

$$
\Phi=\sum_{j=1}^{N} c_{j} p_{j}+R
$$

where the vector $R$ represents an error term.

To estimate the dipole magnitudes, $M$ is chosen greater than N; and a set of values of the p's is found which provides a best fit to the measured surfaco potentiols using a least squares criterion. The p's are found by solving the set of equations (eq. Il of ref. 12):

$$
c_{k}^{t} \Phi=\sum_{j=1}^{N} c_{k}^{t} c_{j} p_{j} \quad(k=1, \ldots N),
$$

where superscript $(t)$ denotes the transpose. An elgorlthm to solve this set of equations is presented in reference 12 .

The above equations may be written more concisely by defining a vector $p$ to represent the set of dipole magnitudes, and by defining an $M$ by $N$ matrix $C$ such that the columns of $C$ are the vectors $C_{1}-C_{N}$. The above set of equations may now be rewritten as

$$
c^{t} \mathrm{Cp}=\mathrm{C}^{t} \Phi \text {. }
$$

Assuming the columns of $C$ are linearly independent, 
the matrix $C^{t} C$ has an inverse; and the solution to this equation $1 \mathrm{~s}$

$$
p=\left(c^{t} c\right)^{-1} c^{t} \Phi
$$

The metrix $\left(c^{t_{C}}\right)^{-1_{C}}{ }^{t}$ is the Penrose generalized inverse of $\mathrm{C}^{13}$ According to Plerce, 14 the goneralized inverse gives an optimum estimate of $p$ under the following conditions: the elements of the nolso vector $R$ have equal varlence, the covarlance of any two different elements is zero, and the nolse variance is vanishingly small compared to the slgnal (Cp).

The effect of nolse on the results is discussed In ref. 12, although not exhaustively. The main conclusion is that if the columns of $c$ aro nearly linearly depondent, the solution will be more sensltive to nolse.

\section{Other investigators have used similar models} which include additional constroints. Barr, et al. used an on-off model in which the magnitude of each dipole is constrained to be either a fixed positive value or zero. 15,16 Lynn, et el. used a model in which the dipolo magnitude is constrained to be nonnegative. 17 While both models have been applied experimentally, neither paper contains an analysis of the effects of noise on the results. In studying epileptic EEG discharges, Schnelder6 modeled the source as a single dipole with random position and ortentetion. The head was modeled as a homogeneous sphere. The procedure used to determine the dipole para- 
meters is as follows: An initial guess is mede for tho three position and throe magnitude components. A parameter to measure the degree of mismatch is deflned, which may be written as

$$
s=\left|w_{m}-w_{c}\right|^{2} /\left|w_{m}\right|^{2}
$$

$W_{m}$ is a column voctor consisting of the messured electrodo potentials, and $W_{c}$ is a column vector of the thooretical potentials which would exist at the eloctrodes due to the assumed dipole.

$W_{m}$ is constant for a particular experiment, and $W_{c}$ may be calculated using an equation originally derived by Wllson and Balley, ${ }^{18}$ so $S$ may be written as a function of the six parameters of the essumed dipole. The differential of this function may be computed, and a now estimate of the dipole parameters is determined by adjusting them so as to decrease $S$. This 1teration is repeated until $s$ becones loss than a prodetermined maximum. Accuracy of the procodure was studied experimentally by taking soreral readings on the same patient, and by simulating a source with added noise. Schneider concludes thet the process can locato a dipole within a volume of sbout two cubic centimeters.

Schnolder reaches the following conclusions about the physical significance of the calculated dipole: EEG anomalies having a nerrow distribution on the scalp usually result from a small anatomic lesion which can be found 
surglcally or with intracerebral recordings. In the case of anomalles with large distributions, a single dipole near the center of the brain provides a good representation In most cases, but this is probably only on olectrical equivalent since high intensities have not been observed doop inside the brain. In some cases the orror , $S$, does not approach zero after many 1terations. Possible reasons for this lack of convergence are that a single dipole is not a good model of the actual source in theso cases, or that there are conditions under which the algorithm does not converge.

In a later paper, 19 Schnelder demonstrates that the single sphere model of the hoad leads to significant orrors, and that for better accuracy the three spheres nodel should be used. 
SCOPE OF THESIS

In this thesis, a new procedure is presented for estimating the location and orientation of a current dipole source given the potentials on the surface of a spberical volume conductor. Original work to be described includes an extension of the theory of the matched filter to functions dofined on a spherical surface and the subsequent application of this result to the problem of loceting a dipole current source.

Simplifications of this locating procedure are also presented which result from resolving the dipole source into three components. The procedure, including simplifications, can be applied oqually well to either the single sphere or three spheres model. The filtering procedure is developed assuming a single dipole is present, but it may be possible to apply the procedure with reduced accuracy if several are present.

Certain notational conventions have been adopted for use in this thesis. For simpliclty, the integral of a function $f(\hat{\partial}, \phi)$ over the surface of a sphere will be written as $\int f(\theta, \phi) d q$. It is to be understood that $\mathrm{dq}=r_{r}^{2} \sin \hat{d} \mathrm{~d} \theta \mathrm{d} \phi$, where $r_{r}$ is the radius of the sphere, and that the limits of integration are $0<\theta<\pi$ and $0<\phi<2 \pi$. To distinguish botween scalars and vectors in abstract 
spaces, the former will be represented by lower case letters and the latter by capitals. Vectors in a three dimensional coordinate sjstem will be represented by lower case letters with a bar (-) above them. Estimated values of parameters will be indicated by placing a caret $(\hat{)})$ above them. 


\section{THE MATCHED FILTER DETECTION PROCESS}

The basic theory of the matched filter will now be described and it will be shown that a matched filter can also be applied to two dimensional functions. It will then be shown that the matched filter can be applied to the problem of determining the location of a current dipole. Finally, some simplifioations to make this locating procedure easier to implement will be described.

A problem which arises in communtcations is to design a filter which will maximize the probability of detecting a pulse in the presence of nolse. If the pulse waveform is $s(t)$, the filter input mas be written as $v(t)=s(t)+n(t)$, where $n$ is random notse. If the impulse response of the filter is $h(-t)$, the filter output is $b(t)=\int_{-\infty}^{\infty} h(\tau-t) v(\tau) d \tau$, by the convolution integral. Assuning $\mathrm{n}$ is white noise, the filter which maximizes the output signal to noise ratio is the matched filter, the impulse response of which is defined by $h(t)=s(t) / c$, where $c$ is an arbitrary constant. 20 The maximum output signal component is then

$$
b(0)=\int_{-\infty}^{\infty} h(T) s(T) d T .
$$

Hirho norms form is $h(t)$. This form has been used to correspond more closely to the definition which will be used in the two dimensional case. 


\section{A. PRELIMINARY DEFIMITIONS AND ASSUMPTIONS}

Before deriving the matched filter for a spherical surface, some preliminary definitions and assumptions will be necessirg. Let vector $\bar{Y}$ represent a point in a three dimensional coordinate system. A sphere of radius $r_{r}$ is then dofined as the set of points for which $|\vec{r}|=r_{r}$. As an extension of the one dimensional case, the observed algnal, $v$, on the spherical surface will be modeled as

$$
\nabla(\bar{r})=s(\bar{r})+n(\bar{r}),|\bar{r}|=r_{r} .
$$

In spherical coordinates, $F$ is specified by the set of parameters $(r, \theta, \not)$, so that the observed potentlal may bo written as

$$
v(\theta, \phi)=s(\theta, \phi)+n(\theta, \phi) \text {, }
$$

since the coordinate $r$ is constent over the surface.

It will be assumed throughout this thesis that all surface potentials are measured with a neutral point used as a reference. In practice, other measurement techniques are often used. These techniques and their posible effects on the accuracy of the estimated dipole parameters will be discussed in chapter $V$.

The function $v(\theta, \phi)$ represonts the voltege observod at overy point on the surfece at a particular Instant of time. The objective of the locating procedure is to find the current dipole which produces a surfaco 
potential most noarly the same as $v(\theta, \phi)$. In tbis initiol derivation $1 t$ will be assumed that $v 1 s$ known at every point. Later, the more practical cose in which the avallable Information consists of a set of samples of $v$ at spocific points on the surfoce will be discussed. The signel component of $v, s(\theta, \phi), 18$ the theoretical surface potential of a current dipole with a speciflc location and orientation. Equations defining s for the single sphere and threo spheros models are evailable in the literoture and have been included in appendix A for reference. For a specific model, 3 is completelg specified by six parameters defining the location, magnitude, and orientation of tho dipole. Ono possible set of parameters congists of the three parameters $(r, \psi, w)$ defining the position and the parameters $(\alpha, \beta, \alpha)$ dofining the orientation and magnitude (seo flg. 1). Alternatively, the orientation and magnitude may be Implicitly specifled by the magnitudes $\left(d_{r}, d_{\theta}, d_{\phi}\right)$ of the dipole components in the directions of the $\overline{8}_{r}, \bar{\theta}_{\theta}$, and $\bar{u}_{\phi}$ unit vectors of the spherical coordinate system. Whon it is desireble to show the dependence of the aignal on the dipole perameters, 3 will be written as $\begin{aligned} \therefore \quad & s(\theta, \phi \mid r, \psi, \omega, \propto, \beta, d), \text { or } \\ & s\left(\theta, \phi \mid r, \psi, \omega, d_{r}, d_{\theta}, d_{\phi}\right),\end{aligned}$

where the vertical bar is used to differentiato between tho surface coordinates and the dipole perameters. Since tho volume conductor is modeled as Iinoar, 


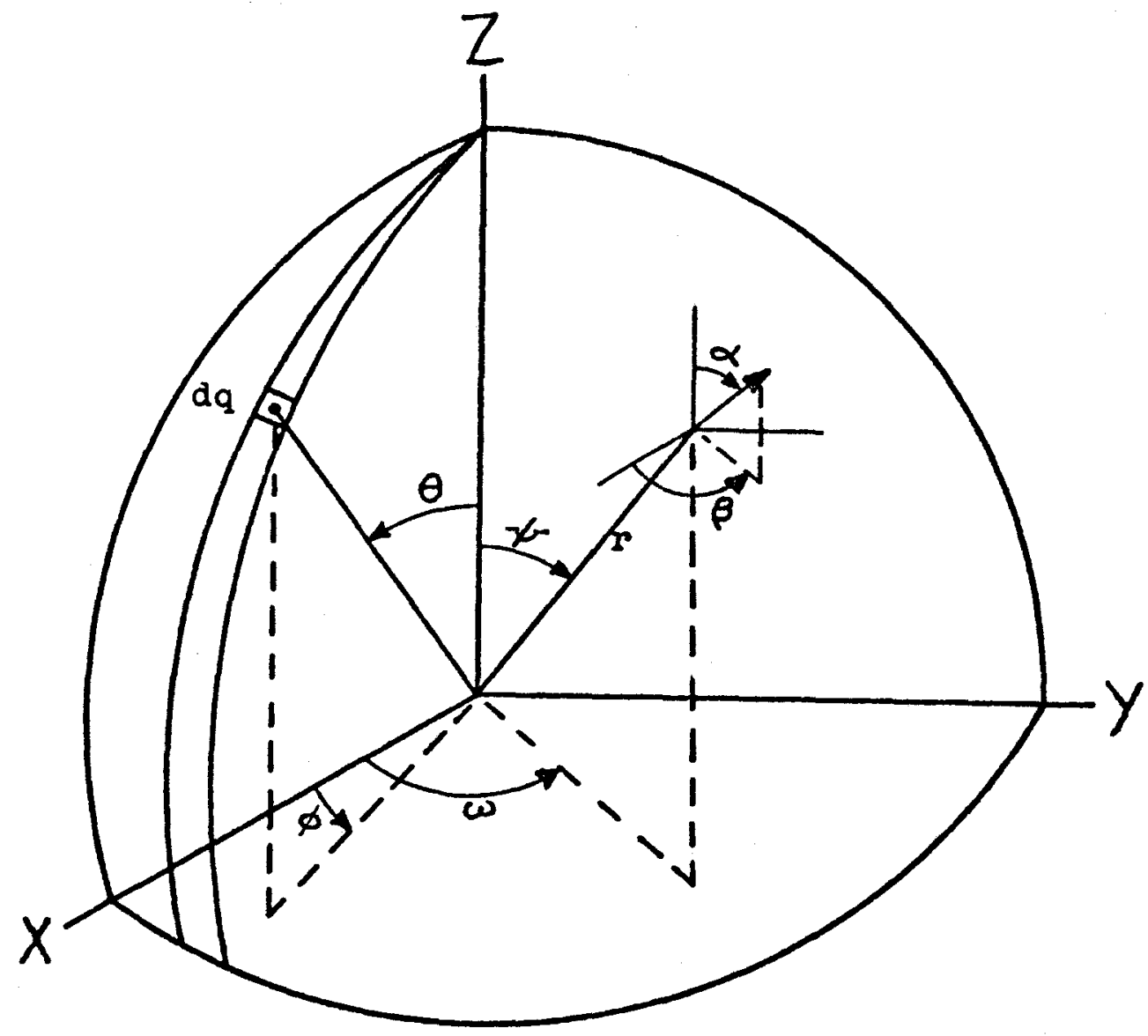

FIGURE 1. The single sphere model of the human head. Coordinates $\theta$ and $\phi$ define the position of an element of area, dq, on the surface, while $\psi, W$, and $r$ define the dipole position. The dipole orlentation is specified by $\alpha$ and $\beta$. 
$s$ is proportional to the dipole magnitude, d; so if function $s_{d}$ is the surface potential distribution which results from a dipole of unit magnitude,

$$
\mathbf{s}(\theta, \phi \mid \mathbf{r}, \psi, \omega, \alpha, \beta, d)=d s_{d}(\theta, \phi \mid r, \psi, \omega, \alpha, \beta) . \quad(I I-2)
$$

In sddition, using the principle of superposition, the surface potential will be the sum of contributions from the three components $\mathrm{d}_{r}, \mathrm{~d}_{\theta}$, and $\mathrm{d}_{\phi}$. Let $s_{r}, s{ }$, and ${ }^{3} \phi$ be the surface potential functions resulting from unit magnitude dipoles oriented in the $\bar{a}_{r}, \bar{a}_{\theta}$, and $\bar{a}_{\phi}$ directions, respectively. Now, $s$ can also be expressed as

$$
\begin{aligned}
& s\left(\theta, \phi \mid r, \psi, \omega, d_{r}, d_{\theta}, d_{\phi}\right)=d_{r} s_{r}(\theta, \phi \mid r, \psi, \omega) \\
& \left.+d_{\theta} s_{\theta}(\theta, \phi \mid r, \psi, \omega)+d_{\phi} s_{\phi}(\theta, \phi \mid r, \psi, \omega) \text { (II }-3\right) \\
& \text { The noise component of } v, n(\theta, \phi), \text { represents }
\end{aligned}
$$

all sources of error, including nolse in the ampliflers, other electrical activity of the brain, and inaccuracios of the model.

Two types of delta functions will be needed at this point in order to describe the noise mathematically. The ordinary one dimensional delta will be represented by $\delta_{1}(t)$, while $\hat{O}_{3}(\bar{r})$ will be a two dimensional delta function defined on the surface of a sphere of radius $r_{r}$. As in the one dimensional case, the notse will be modeled as white. In one dimension, the noise is white if $n(t)$ is statistically stationary and has the properties 


$$
\begin{aligned}
& E[n(t)]=0, \text { and } \\
& E\left[n\left(t_{1}\right) n\left(t_{2}\right)\right]=k^{2} \delta_{1}\left(t_{2}-t_{1}\right),
\end{aligned}
$$

where E represents the expectation operator, and $k$ is a constent. For the sphorical case, these propertios become

$$
\begin{aligned}
& E[n(\bar{r})]=0, \text { ond } \\
& E\left[n\left(\bar{r}_{1}\right) n\left(\bar{r}_{2}\right)\right]=k^{2} \delta_{s}\left(\bar{r}_{2}-\bar{r}_{1}\right) .
\end{aligned}
$$

Care must be taken in expressing the delta function in spherical coordinates.

In any coordinate system delta must have tho rollowing properties: 21

$$
\begin{aligned}
& \delta_{S}\left(\bar{r}-\bar{r}_{0}\right)=0 \text { if } \bar{r} \neq \bar{r}_{0}, \text { and } \\
& \int_{s}\left(\bar{r}-\bar{r}_{0}\right) d q=1,
\end{aligned}
$$

where $\Gamma_{0}$ is a point on the sphere, and $\bar{F}$ is the position of $d q$. In spherical coordinates this integral bocomes

$$
\int_{0}^{2 \pi} \int_{0}^{\pi} \delta\left(\bar{r}-\bar{s}_{0}\right) r_{r}^{2} \sin \theta \mathrm{d} \theta \mathrm{d} \phi \text {. }
$$


It is easily seen thet the value of this integral is one if

$$
\delta_{s}\left(\bar{r}-\bar{r}_{0}\right)=\frac{\delta_{1}\left(\theta-\theta_{0}\right) \delta_{1}\left(\phi-\phi_{0}\right)}{r_{r}^{2} \sin \theta}
$$

The properties assumed for the noise can then be written as

$$
\begin{aligned}
E[n(\bar{r})]=0, \text { and } & \\
E\left[n\left(\bar{r}_{1}\right) n\left(\bar{r}_{2}\right)\right] & =k^{2} \delta_{s}\left(\bar{r}_{2}-\bar{r}_{1}\right) \\
& =k^{2} \frac{\delta_{1}\left(\theta_{2}-\theta_{1}\right) \delta_{1}\left(\phi_{2}-\not_{1}\right)}{r_{r}^{2} \sin \theta} .
\end{aligned}
$$

Using this definition the sifting property of the delta function also holds in spherical coordinates. That is, for any function $f(\bar{r})$ the following relationshlp holds:

$$
\int f(\bar{r}) \delta_{S}\left(\bar{r}-\bar{r}_{0}\right) d q=f\left(\bar{r}_{0}\right) \text {. }
$$

This relationship can easily be proven by writing

the integral as

$$
\int_{0}^{2 \pi} \int_{0}^{\pi} f(\theta, \phi) \frac{\delta\left(\theta-\theta_{0}\right) \delta\left(\phi-\phi_{0}\right)}{r_{r}^{2} \sin \theta} r_{r}^{2} \sin \theta d \theta d \phi .
$$

which reduces to

$$
\int_{0}^{2 \pi} \int_{0}^{\pi} \rho(\theta, \phi) \delta_{1}\left(\theta-\theta_{0}\right) \delta_{1}\left(\phi-\phi_{0}\right) \mathrm{d} \theta \mathrm{d} \phi \text {. }
$$

Two applications of the sifting property of the one dimensional delta function produce the desired result. 


\section{B. DERIVATION OF THE SPHERICAL MATCHED FI LTER}

The matched filter may be derived for a spherical surface using a method similar to that applied in the one dimensional case. 20 The filter output is

$$
b=\int h(\theta, \phi) v(\theta, \not) d q \text {, }
$$

whlch consists of a signal component

$$
p=\int h(\theta, \phi) s(\theta, \phi) d q
$$

and a nolse component

$$
\mathrm{u}=\int \mathrm{h}(\theta, \phi) \mathrm{n}(\theta, \phi) \mathrm{dq} \text {. }
$$

The matched filter problem is to maximize the output peak signal to average noise power ratio $\eta=p^{2} / E\left(u^{2}\right)$. This problem is equivalent to maximizing the rotio $p^{2} /\left[w E\left(u^{2}\right)\right]$; where w equals $\int s^{2} d q$, the total energy in the signal, which is a fixed value for a particular instant of time.

To demonstrate that the matched filter (a rilter whose impulse response has the samo shape as the signal) maximizes $\eta$, it will be necessary to evaluate $E\left(u^{2}\right)$. This quantity may be evaluated as shown in ref. $22, p .324$. Let $u_{1}=\int n(F) h_{1}(\bar{r}) d q$ and $u_{2}=\int n(\bar{r}) h_{2}(\bar{r}) d q$. Then if $d q_{i}$ is the element of area

\footnotetext{
The torm energy as used here is the integral of voltage squared (power) over the surface, which is analagous to the definition for time slgnals as the integral of voltage squared over time.
} 
at point $\bar{r}_{1}$.

$$
\begin{aligned}
E\left(u_{1} u_{2}\right) & =E\left[\int n\left(\bar{r}_{1}\right) h_{1}\left(\bar{r}_{1}\right) d q_{1} \int n\left(\bar{r}_{2}\right) h_{2}\left(\bar{r}_{2}\right) d q_{2}\right] \\
& =E\left\{\int\left[\int n\left(\bar{r}_{1}\right) n\left(\bar{r}_{2}\right) h_{1}\left(\bar{r}_{1}\right) d q_{1}\right] h_{2}\left(\bar{r}_{2}\right) d_{2}\right\} .
\end{aligned}
$$

Taking the expected value inside the integral, and assuming white noise, the equation becomes

$$
E\left(u_{1} u_{2}\right)=k^{2} \int\left[\int \delta\left(\bar{r}_{2}-\bar{r}_{1}\right) h_{1}\left(\bar{r}_{1}\right) d q_{1}\right] h_{2}\left(\bar{r}_{2}\right) d q_{2} \text {. }
$$

Applying the sifting property of the delta function,

this equation reduces to

$$
E\left(u_{1} u_{2}\right)=k^{2} \int h_{1}\left(\bar{r}_{2}\right) h_{2}\left(\bar{r}_{2}\right) d q_{2} \text {. }
$$

If $u_{1}=u_{2}=u$, this equation reduces to

$$
\begin{aligned}
& E\left(u^{2}\right)=k^{2} \int b^{2}(\bar{r}) d q \\
& \text { Now, } \\
& \frac{\eta}{w}=\frac{p^{2}}{w E\left(u^{2}\right)}=\frac{\left[\int h s d q\right]^{2}}{\left[\int s^{2} d q\right]\left[k^{2} \int h^{2} d q\right]} .
\end{aligned}
$$

Schwarz's inequality states that $\left[\int \mathrm{hsdq}\right]^{\hat{C}^{2}} \equiv \int \mathbf{s}^{2} \mathrm{dq} \int \mathbf{b}^{2} \mathrm{dq}$. Substituting this inequelity in the above numerator and cancelling terms gives $\eta / w \leq 1 / k^{2}$, or $\eta \leq w / k^{2}$. Equality bolds in Schwarz's inequelity, and $\eta$ is moximum, only when:

$$
h(\theta, \not)=s(\theta, \phi) / c \text {. }
$$

Therefore, as in the one dimensionsl case, the matched rilter produces the greatest posible value of the output signal to nolse ratio, $\eta$.

$$
\begin{aligned}
\text { Th1s maximum value of } \eta \text { is then } \\
\eta_{m}=\left(w / k^{2}\right)=\left(1 / k^{2}\right) \int[\operatorname{ch}(\theta, \not)]^{2} d q \\
=\left(c^{2} / k^{2}\right) \int h^{2}(\theta, \not) d q .
\end{aligned}
$$


To apply the matched filter to the problen of locating a current dipole, it will be convenient to define a real vector space consisting of functiona of $\theta$ and $\phi$, with the inner product of two elements $h$ and $v$ deflned as $\langle h \mid v\rangle=\int h r d q$. The cosine of the anglo betwoen two elements, $\gamma$, is then definod as 23

$$
\gamma=\left[\int h v d q\right] /\left[\int h^{2} d q \int v^{2} d q\right]
$$

Note that Schwarz's inequality implies $|\gamma| \leq 1$. (II-15b) Using the subscript 0 to indicate the location and orientation coordinates of the actual dipole, the surface potential is

$$
v(\theta, \phi)=d s_{d}\left(\theta, \phi \mid r_{0}, \psi_{0}, \omega_{0}, \alpha_{0}, \beta_{0}\right)+n(\theta, \phi) .
$$

The filter function $b$ will be chosen proportional to the theoretical surface potential of an arbitrary dipole:

$h h(\theta, \phi \mid r, \psi, \omega, \alpha, \beta)=s_{d}(\theta, \phi \mid r, \psi, \omega, \alpha, \beta) / c(r, \psi, \omega, \alpha, \beta)$

The relationshlp between $h$ and $s_{d} w 1 l l$ be defined such that

$$
\int h^{2}(\theta, \phi) d q=1 \text {, }
$$

and therefore

$$
c(r, \psi, \omega, \alpha, \beta)=\left[\int s_{d}^{2}(\theta, \phi \mid r, \psi, \omega, \alpha, \beta) d q\right]^{\frac{1}{2}} .
$$

As a rosult of this definition

$$
\gamma=\frac{\int h(\theta, \phi) v(\theta, \phi) d q}{\left[\int v^{2}(\theta, \phi) d q\right]^{2}} .
$$

since $\nabla$ bas a random component, $\gamma$ is a randor process 
which depends on the parameters of the arbltrary dipole:

$$
\gamma(r, \psi, \omega, \alpha, \beta)=\frac{\int h(\theta, \phi \mid r, \psi, \omega, \alpha, \beta) v(\theta, \phi) d q}{\left[\int v^{2}(\theta, \phi) d q\right]^{\frac{1}{2}}}
$$

If the assumption $1 \mathrm{~s}$ made that the nolse is negligible, $\nabla=s=d s_{d}$, and $\gamma$ reduces to the deterministic function

$$
\begin{aligned}
& \gamma(r, \psi, \omega, \alpha, \beta)= \\
& \frac{\int h(\theta, \phi \mid r, \psi, \omega, \alpha, \beta) s_{d}\left(\theta, \phi \mid r_{0}, \psi_{0}, \omega_{0}, \alpha_{0}, \beta_{0}\right) d q}{c\left(r_{0}, \psi_{0}, \omega_{0}, \alpha_{0}, \beta_{0}\right)}
\end{aligned}
$$

Now $b$ and $s_{d}$ will be linesrly dependent, and therefore $r$ will equal one, when the five parameters of the assumed dipole correspond to those of the actual dipole; that is, $\gamma$ is one when $r=r_{0}, \psi=f_{0}, w=w_{0}, \alpha=\alpha_{0}$. and $\beta=\beta_{0}$. The dipole position and orientation may therefore be found by finding the set of parameter values ( $r, \psi$, $(, \alpha, \beta)$ for which $\gamma$ attains 1 ts maximum value of one. If the noise is small but not negligible, it is to be expected that a maximum volue of $\gamma$ will still occur when 1 ts arguments are noar the actual dipole position and orlentation. The set of parameters whicb maximizes $\gamma$ then provides an estimate of the actual dipole parameters.

The cosine, $r$, is proportional to the matched fliter output since $\int v^{2} d q$ is constant for a particular experiment and $\int \mathrm{h}^{2} \mathrm{~d} q$ is constant by definition. Combining equations II-8 and II-20, 


$$
\begin{aligned}
b(r, \psi, \omega, \alpha, \beta) & =\int h(\theta, \phi \mid r, \psi, \omega, \alpha, \beta) v(\theta, \phi) d q \\
& =\gamma(r, \psi, \omega, \alpha, \beta)\left[\int v^{2}(\theta, \phi) d q\right]^{\frac{1}{2}}
\end{aligned}
$$

For purposes of determining the dipole location and orlentation, maximizing $b$ is therefore equivalent to $\operatorname{maximizing} \gamma$. 


\section{SEARCH PROCEDURE DEVELOPMENT}

The straightforward approach to determining the source position and orlentation would be to divide the range of each dipole paremeter into segments, evaluato $b$ for all possible combinations, and seloct the set of values for which b is a maximum. However, since five parameters are involved, the number of calculations required becomes probibltive if the increments are chosen small enough to obtain reasonable accuracy.

The number of calculations required may be reduced by modifying the above algorithm as follows. For a specific set of position coordinates $\left(r_{1}, \psi_{1}, \omega_{1}\right)$, find the set of orlentation parameters which maximizes $b$. Repeat the procodure for all locations in the sphere and solect the locetion and corresponding orlentation for which $b$ is a maximum. The advantage of this algorithm is that, as will now be shown, the desired orlontation for a given locetion may be determined with a small smount of computation, and without using an iterative procedure.

In eq. II-17, the filter response function, $h$, was defined to be proportional to the surface potential as expressed in eq. II-2. However, in the derivation 
which follows, an equivalent definition based on eq. II-3

will be more convenient.

$$
\begin{aligned}
& \text { Repeating oq. II-3, } \\
& s\left(\theta, \phi \mid r, \psi, \omega, d_{r}, d_{\theta}, d_{\phi}\right)=d_{r} s_{r}(\theta, \phi \mid r, \psi, \omega) \\
& +d_{\theta}{ }^{\prime}(\theta, \phi \mid r, \psi, \omega)+d_{\psi} \psi_{\psi}(\theta, \phi \mid r, \psi, \omega) .
\end{aligned}
$$

The matched filter will now be defined proportional to the dipole surface potential as defined in eq. II-3.

$$
\begin{aligned}
& h\left(\theta, \phi \mid r, \psi, w, d_{r}, d_{\theta}, d_{\phi}\right)= \\
& \quad\left[a_{r} s_{r}(\theta, \phi \mid r, \psi, \omega)+d_{\theta} \theta(\theta, \phi \mid r, \psi, w)\right. \\
& \left.+d_{\phi} \phi(\theta, \phi \mid r, \psi, \omega)\right] / c\left(r, \psi, w, d_{r}, d_{\theta}, d_{\phi}\right)
\end{aligned}
$$

since the function $s_{d}$, which forms the numerator

of eq. II-17, is defined to be the surface potential

produced by a dipole with unit magnitude, the numerator

of eq. II-24a is equal to $s_{d}$ if

$$
\left[d_{r}^{2}+d_{\theta}^{2}+d_{\phi}^{2}\right]^{\frac{1}{2}}=1
$$

To determine the value of $c$, squaro both sides of the equation and integrate over the surface, giving

$$
\begin{aligned}
& \begin{array}{l}
\left.\int \mathrm{h}^{2} \theta, \phi \mid r, \psi, \omega, \mathrm{d}_{\mathrm{r}}, \mathrm{d} \partial, \mathrm{d} \phi\right) \\
{\left[\mathrm{d}_{\mathrm{r}}^{2} \int_{r}^{2}(\theta, \phi \mid r, \psi, \omega) \mathrm{dq}\right.}
\end{array} \\
& +2 \mathrm{~d}_{r} \mathrm{~d} \theta \int_{s_{r}}(\theta, \phi \mid r, \psi, \omega) s_{\theta}(\theta, \phi \mid r, \psi, \omega) d q \\
& +2 \mathrm{~d}_{\mathrm{r}} \mathrm{d} \phi \int \mathrm{s}_{\mathrm{r}}(\theta, \phi \mid \mathrm{r}, \psi, \omega) \mathrm{s}_{\phi}(\theta, \phi \mid r, \psi, \omega) \mathrm{da} \\
& +\mathrm{d}_{\theta}^{2} \int \mathrm{s}_{\theta}^{2}(\theta, \phi \mid \mathrm{r}, \psi, \omega) \mathrm{d} \mathrm{q} \\
& +2 \mathrm{~d} \theta \mathrm{d} \phi \int \mathrm{s} \theta(\theta, \phi \mid \mathrm{r}, \psi, \omega) \mathrm{s} \phi(\theta, \neq \mid \mathrm{r}, \psi, \omega) \mathrm{dq} \\
& \left.+\mathrm{d}_{\phi}^{2} \int_{\psi}^{2}(\theta, \phi \mid r, \psi, \omega) \mathrm{dq}\right] / \mathrm{c}^{2}\left(\mathrm{r}, \psi, \omega, \mathrm{d}_{r}, \mathrm{~d}_{\theta}, \mathrm{d}_{\phi}\right) \text {. (II-25) }
\end{aligned}
$$

potentials produced by the three component dipoles are mutually orthogonal. As a result, the cross terms of 
the above equation are equal to zero, and this equation

reduces to

$$
\begin{aligned}
& \int \mathrm{h}^{2}\left(\theta, \phi \mid r, \psi, \omega, \mathrm{d}_{\mathrm{r}}, \mathrm{d} \theta, \mathrm{d} \phi\right) \mathrm{dq}= \\
& {\left[d_{r}^{2} \int_{r}^{2}(\theta, \phi \mid r, \psi, w) d q\right.} \\
& +\mathrm{d}^{2} \int_{0}^{2} s_{\theta}^{2}(\theta, \phi \mid \mathrm{r}, \psi \mathrm{d}) \mathrm{dq} \\
& +\mathrm{d}_{\phi}^{2}\left[\mathrm{~s}_{\phi}^{2}\left(\theta, \phi \mid r, \psi_{w}\right) \mathrm{dq}\right] / \mathrm{c}^{2}\left(r, \psi, w_{,} \mathrm{d}_{r}, \mathrm{~d}_{\theta}, \mathrm{d}_{\phi}\right) \text {. }
\end{aligned}
$$

The left side of this equation is equal to one, by equation II-18; so, making this substitution in eq. II-26 and rearranging terms gives

$$
\begin{aligned}
& c\left(r, \psi, w, d_{r}, d_{\theta}, d_{\phi}\right)=\left[d_{r}^{2} \int s_{r}^{2}(\theta, \phi \mid r, \psi, w) d q\right. \\
& \left.+d_{\theta}^{2} \int s_{\theta}^{2}(\theta, \phi \mid r, \psi, \omega) d q+d_{\phi}^{2} \int s_{\phi}^{2}(\theta, \phi \mid r, \psi, \omega) d q\right]^{\frac{2}{2}} .
\end{aligned}
$$

For later use, a set of three fliters will be

defined, one matched to each component of s. Three constants, cr, c $\theta$, and $c \phi$, will first be defined as follows:

$$
\begin{aligned}
& c_{r}\left(r, \psi(\omega)=\left[\int s_{r}^{2}\left(\theta, \phi \mid r, \psi_{d}\right) \mathrm{dq}\right]^{\frac{1}{2}}\right. \\
& c_{\theta}\left(r, \psi_{d}\right)=\left[\int s_{\theta}^{2}(\theta, \phi \mid r, \psi, \omega) \mathrm{dq}\right]^{\frac{1}{2}} \\
& c_{\phi}\left(r, \psi_{d}\right)=\left[\int s_{\phi}^{2}(\theta, \phi \mid r, \psi, \omega) \mathrm{dq}\right]^{\frac{1}{2}}
\end{aligned}
$$

The three filter response functions $h_{r}$, ${ }{ }_{\theta}$, and $h_{\phi}$, which are matched to the $\bar{a}_{r}, \bar{a}_{\theta}$, and $\bar{\theta}_{\phi}$ dipole components, respectively, may now be defined as

$$
\begin{aligned}
& h_{r}(\theta, \phi \mid r, \psi, \omega)=s_{r}(\theta, \phi \mid r, \psi, \omega) / c_{r}(r, \psi, \omega) \\
& { }^{n_{\theta}}(\theta, \phi \mid r, \psi ; \omega)=s_{\theta}(\theta, \phi \mid r, \psi, w) / c_{\theta}\left(r, \psi_{m}\right) \\
& { }{ }_{\phi}(\hat{\theta}, \phi \mid r, \psi / \omega)=s_{\phi}\left(\theta, \phi \mid r, \psi_{\alpha}\right) / c_{\phi}\left(r, \psi_{\alpha}\right)
\end{aligned}
$$

With $c_{r},{ }^{c} \theta$, and ${ }^{c} \phi$ as defined in eq. II-28, it is obvious 
thet these response functions conform to the constraint, expressed in eq. II-18, that the integral of the square of each response function be equal to unity.

By substituting equations II-28 into II-27, that equation becomes

$$
\begin{gathered}
c\left(r, \psi, \omega, a_{r}, a_{\theta}, d_{\phi}\right)=\left[d_{r}^{2} c_{r}^{2}(r, \psi, w)\right. \\
\left.+d_{\theta}^{2} c_{\theta}^{2}(r, \psi, \omega)+d_{\phi}^{2} c_{\phi}^{2}(r, \psi, \omega)\right]^{\frac{2}{2}} .
\end{gathered}
$$

Next, derine the variables

$$
\begin{aligned}
& d_{r}^{\prime}=d_{r} c_{r}\left(r, \psi_{r}\right) / c\left(r, \psi, \omega, d_{r}, a_{\theta}, d_{\phi}\right) \\
& d_{\theta}^{\prime}=d_{\theta} c_{\theta}\left(r, \psi_{,}\right) / c\left(r, \psi, \omega, d_{r}, d_{\theta}, d_{\phi}\right) \\
& d_{\phi}^{\prime}=d_{\phi} \phi(r, \psi, \omega) / c\left(r, \psi, \omega, d_{r}, d_{\theta}, d_{\phi}\right) .
\end{aligned}
$$

Solving these equations for $\mathrm{d}_{r}$, $\mathrm{d} \theta$, and $\mathrm{d} \phi$, substituting into eq. II-30, and dividing by $c$, it is found that

$$
\left(d_{r}^{\prime}\right)^{2}+\left(d_{\theta}^{\prime}\right)^{2}+\left(d_{\phi}^{\prime}\right)^{2}=1 \text {. }
$$

The reletionsbip between the filter response function, $h$, and the three component response functions may be determined by solving equations II-29 for sp' s $\theta$, and s $\phi$, solving equations II-3I for $\mathrm{d}_{r}, \mathrm{~d}_{\theta}$, and $\phi$, and substituting into equation II-24. After cancelling terms, it is found that

$$
\begin{gathered}
h\left(\theta, \phi \mid r, \psi, w, a_{r}, d_{\theta}, d_{\phi}\right)= \\
d_{r}^{\prime} h_{r}\left(\theta, \phi \mid r, \psi_{,}\right) \\
+d_{\theta}^{\prime} h_{\theta}\left(\theta, \phi \mid r, \psi_{, \omega}\right) \\
+d_{\phi}^{\prime} h^{\prime}\left(\theta, \phi \mid r, \psi_{w}\right) .
\end{gathered}
$$

To determine the dipole orientation which maximizes tho fliter output for a given position, conbine 
eq. II -8 and eq. II-33, to obtoin

$$
\begin{aligned}
& b\left(r, \psi, \omega, d_{s}, d_{\theta}, d_{\phi}\right)= \\
& d_{r}^{\prime} \int h_{r}(\theta, \not \mid r, \psi, \omega) v(\theta, \phi) d q \\
&+ d_{\theta}^{\prime} \int h_{\theta}(\theta, f \mid r, \psi, \omega) v(\theta, \phi) d q \\
&+ d_{\phi}^{\prime} \int h_{\phi}(\theta, \not \mid r, \mathcal{F}, \omega) v(\theta, \not) d q .
\end{aligned}
$$

This result ray be thought of as the inner product of the two vectors

$$
D^{\prime}=\left[d_{r}^{\prime}, d_{\theta}^{\prime}, d_{\phi}^{\prime}\right]^{t}
$$

and

$$
B=\left[\begin{array}{l}
b_{r} \\
b_{\theta} \\
b_{\phi}
\end{array}\right]=\left[\begin{array}{l}
\int h_{\gamma}(\theta, \phi) v(\theta, \phi) d q \\
\int h_{\theta}(\theta, \phi) v(\theta, \phi) d q \\
\int h_{\phi}(\theta, \phi) v(\theta, \phi) d q
\end{array}\right] .
$$

For a given function $v(\theta, \not)$ and set of coordinates $(r, f, \omega)$, the values of $b_{r}, b_{\theta}$, and $b_{\phi}$ may bo calculated using eq. II-36; and the megnitude of vector $D^{\prime}$ is one, from oq. II-32. Therefore, by Schwarz's Inequalitf, $b$ in eq. II-34 is maximum when vectors $D^{\prime}$ and $B$ are linearly dependent. That is, the value of $D^{\prime}$ which maximizes the fllter output, $b$, for an assumed dipole position is

$$
D_{m}^{\prime}=\mathrm{BB}
$$

where $g$ is a constant of proportionality. Since

$$
\begin{aligned}
& \left|D_{m}^{\prime}\right|=1, B|B|=1 \text {, or } B=1 /|B| \text {. So, } \\
& D_{m}^{\prime}=B /|B| \text {. }
\end{aligned}
$$

Substituting this result into eq. II- 34 , the maximum filter output, $b_{m}$, for an assumed dipolo location $(r, \gamma, \omega)$ is 


$$
b_{m}(r, \not, \omega)=D_{m}^{\prime} \cdot B=(B \cdot B) /|B|=|B| \text {. }
$$

Equation II-38 gives in closed form the maximum value of $b$ over the parameters $\alpha$ and $\beta$ with $r, \psi$, and $w$ fixed. To determine the dipole location it is necosary to maximlze $b$ over the parameters $r, z^{r}$, and $\omega$ also. While this operation could be performed using an iterative procedure like that used by Schneider, the maximum can also be epproximated by evaluating $b_{m}$ for discrete points In the sphere and solecting the point for which $b_{m}$ is a maximum.

After the dipole locetions are determined, the magnftudes of the component dipoles mag be calculated using equations which will now be cerfved.

Substitute $\nabla=8+n$ into eq. II -36 with the 81gnal, 8, represented as in eq. II-3. If the ectual dipole location is $\bar{r}_{0}=\left(r_{0}, f_{0}, \omega_{0}\right)$, the equation for $b_{p}$ at that point now becomes

$$
\begin{aligned}
& b_{r}\left(\bar{r}_{0}\right)=\int h_{r}\left(\theta, \phi \mid \bar{r}_{0}\right)\left[d_{r} s_{r}\left(\theta, \phi \mid \bar{r}_{0}\right)\right. \\
& \left.\left.\quad+d_{\theta} s_{\theta}\left(\theta, \phi \mid \bar{r}_{0}\right)+d_{\phi s}\left(\theta, \phi \mid \bar{r}_{0}\right)+n(\theta, \phi)\right] d q \cdot \text { (II }-39\right)
\end{aligned}
$$

Since, as previousig stated, the surfaco potentials due to the three component dipoles are mutually orthogonal, this equation reduces to

$$
\begin{aligned}
b_{r}\left(\bar{r}_{0}\right) & =d_{r} \int h_{r}\left(\theta, \phi \mid \bar{r}_{0}\right) s_{r}\left(\theta, \phi \mid \bar{r}_{0}\right) d q \\
& +\int h_{r}\left(\theta, \phi \mid \bar{r}_{0}\right) n(\theta, \phi) d q .
\end{aligned}
$$

Substituting eq. II-28 for $s_{r}$ and solving for $d_{r}$ jields:

$$
d_{r}=\frac{b_{r}\left(\bar{r}_{0}\right)}{c_{r}\left(\bar{r}_{0}\right)}-\frac{\int h_{r}\left(\theta, \phi \mid \bar{r}_{0}\right) n(\theta, \phi) d q}{{ }^{c}\left(\bar{r}_{0}\right)}
$$

In the absence of nolse, the ratio of $b_{x}$ to o $r$ 
gives the correct value of $d_{r}$. When tho nolse is nonzero, this ratio provides an estimate of $\mathrm{d}_{\mathrm{r}}$ with the error given by the last term of oq. II-4I.

The estimated values of the othor two components may bo obtainod in a similar mannor, giving

$$
\begin{aligned}
& \hat{d}_{r}=b_{r}\left(\bar{r}_{0}\right) / c_{r}\left(\bar{r}_{0}\right) \\
& \hat{d}_{\theta}=b_{\theta}\left(\bar{r}_{0}\right) / c_{\theta}\left(\bar{r}_{0}\right) \\
& \hat{d}_{\phi}=b_{\phi}\left(\bar{r}_{0}\right) / c_{\phi}\left(\bar{r}_{0}\right) .
\end{aligned}
$$

The orror in each component is then

$$
\begin{aligned}
& \left(\hat{d}_{r}-d_{r}\right)=\left[\int h_{r}\left(\theta, \phi \mid \bar{r}_{0}\right) n(\theta, \phi) d q\right] / c_{r}\left(\bar{r}_{0}\right) \\
& \left(\hat{d}_{\theta}-d_{\theta}\right)=\left[\int h_{\theta}\left(\theta, \phi \mid \bar{r}_{0}\right) n(\theta, \phi) d q\right] / c_{\theta}\left(\bar{r}_{0}\right) \\
& \left(\hat{d}_{\phi}-d_{\phi}\right)=\left[\int h_{\phi}\left(\theta, \phi \mid \bar{r}_{0}\right) n(\theta, \phi) d q\right] / c_{\phi}\left(\bar{r}_{0}\right)
\end{aligned}
$$

In summary, the sireplified locating procedure consists of the following operations:

(1) Divide the renge of esch of the position coordinates $r, \gamma$, w into sogments.

(2) For each set of position coordinates evaluate $b_{p}, b_{\theta}, b_{\phi}$, and $b_{m}$ using equations II- 36 and II-38. (3) Compare the resulting values of $b_{m}$ and solect the set of coordinates whlch produced the largest magnitude. This point is the estimated dipole position.

(4) The component magnitudes may then be calculated using $\theta$. II -42 .

सrinese oquations asume the dipole location is known exactiy. In genersl, the nolso can blso ceuse error in the estimated dipole position which will in turn cause more orror in the regnitudes calculated with eq. II- 42 . 


\section{DI SCRETE CASE}

In a practical application the surface potential. v, is known only at a finite set of points, corresponding to the olectrodo positions. The matched filter detection procedure will therefore be developed for the discrete c8se 8150.

From cquation II-l, the surface potentials at m electrode locotions are

$$
v\left(\theta_{1}, \phi_{1}\right)=s\left(\theta_{1}, \phi_{1}\right)+n\left(\theta_{1}, \phi_{1}\right), \quad 1=1, \ldots \ldots .
$$

In addition, from eq. II-2,

$$
s\left(\theta_{1}, \phi_{1} \mid r, \psi, \omega, \alpha, \beta, d\right)=\mathrm{ds}_{\mathrm{d}}\left(\theta_{1}, \phi_{1} \mid \mathrm{r}, \psi, \omega, \alpha, \beta\right), i=1, \ldots \mathrm{m} .
$$

Letting $V, S, S$, and $N$ bo $m$ dimensional column vectors corresponding to functions $v, s, s_{d}$, and $n$, respectively, these two sets of equations may be expressed as

$$
\begin{aligned}
& v=s+r \\
& s(r, \psi, \omega, \alpha, \beta, d)=\alpha s_{d}(r, \psi, \omega, \alpha, \beta)
\end{aligned}
$$

For this vector space, eq. II- 15 for the cosine of the angle between two vectors, $B$ and $V, 18$ replaced by

$$
\gamma=\frac{H^{t} V}{|H||V|}: \quad|\gamma| \leq 1 \text {. }
$$

As in the continuous case, applying Scbwarz's inequallty. $\gamma$ is equal to one when $H$ is proportional to $S$, sssuming that the noise is negligiblo. Tho filter responso voctor, H, For a dipole with a given magnitude and orientation 
is therefore proportional to the surface potential vector, $S$, of that dipole. Corresponding to eq. II-18, the constant of proportionality will be chosen such that

$$
|\mathrm{H}|=1 \text {. }
$$

Therefore, define the constant c for the discrete case as

$$
c(r, \psi, \omega, \alpha, \beta)=\left|s_{d}(r, \psi, \omega, \alpha, \beta)\right| \text {. }
$$

Eq. II -46 is then satisfied if $\mathrm{H}$ is defined as

$$
H(r, \psi, \omega, \alpha, \beta)=s_{d}(r, \psi, \omega, \alpha, \beta) / c(r, \psi, \omega, \alpha, \beta) \text {. }
$$

Eq. II- 45 then becomes

$$
r=H^{t} \mathrm{~V} /|\mathrm{V}| \text {. }
$$

As in the continuous case, the magnitude of $y$

is constant for a given set of data, so the metched filter output, $b$, may be defined as tho numerator of oq. II -48 . Therefore, eq. II-22 for the discrete case bocomes

$$
\begin{aligned}
b(r, x ; \omega, \alpha, \beta) & =[H(r, f, \omega, \alpha, \beta)]^{t_{V}} \\
& =\gamma(r, f ; \omega, \alpha, \beta)|v| .
\end{aligned}
$$

The sinplificetions dereloped in section $C$

may be applied to the discrete case as well. Equations II-23 through II-25 of section $C$ are replaced by tho following:

$$
\begin{aligned}
& s\left(r, \psi, \omega, d_{r}, d_{\theta}, d_{\phi}\right)=a_{r} s_{r}\left(r, \psi_{,}\right) \\
& +{ }^{a} \theta^{s} \theta^{\left(r, \psi_{o} \omega\right)+\alpha_{\phi} \phi\left(r, \psi_{d}\right)} \\
& \mathrm{H}\left(\mathrm{r}, \psi, \omega, \mathrm{a}_{r}, \mathrm{~d}_{\theta}, \mathrm{a}_{\phi}\right)=\left[\mathrm{d}_{r} \mathrm{~s}_{r}(r, \psi, \omega)+\mathrm{d}_{\theta} \mathrm{s}_{\theta}(r, \psi, w)\right. \\
& +\alpha_{\phi} \phi\left(r, \psi_{\alpha}(N)\right] / c\left(r, \psi, W, \alpha_{r},{ }^{a} \theta, d_{\phi}\right)
\end{aligned}
$$




$$
\begin{aligned}
& H^{t_{H}}=\int d_{r}^{2} s_{r}^{t} s_{r}+2 d_{r} d{ }^{d} s_{r}^{t} s_{\theta}+2 d_{r}{ }^{d} \phi_{r}^{s_{r}^{t}} s_{\phi} \\
& \left.+d_{\theta}^{2} s_{\theta}^{t} s_{\theta}+2 d \theta^{d} \phi^{s} \theta^{t} \phi+d_{\phi}^{2} s^{t} s_{\phi}\right] / c^{2}
\end{aligned}
$$

At this point a possible problen arises. Equation II-26 ut12ized the fact that the functions $b_{f^{\prime}} b_{\theta}$, and $b_{\phi}$ ore mutually orthogonsl oper the surfice. In the discrete e case, there 1 s no guarantes that $\mathrm{H}_{r}, \mathrm{H}_{\theta}$, and $\mathrm{B}_{\phi}$ will be exactly mutually orthogonal. It 18, horever, to bo expected that these three vectors will be approximately orthogonal if a large number of electrodes are used and they are evenly distributed over the surface.

$$
\text { Based on the assumption that } \mathrm{H}_{r}, \mathrm{H}_{\theta} \text {, and } \mathrm{H}_{\phi} \text { are }
$$
orthogonal, equations II-26 through II-38 reg be replaced by:

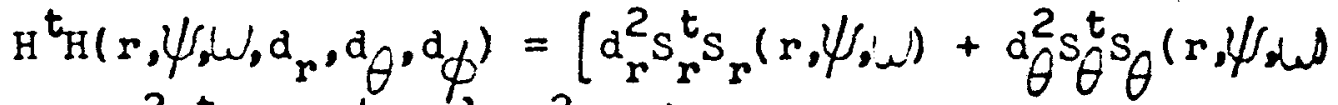

$$
\begin{aligned}
& \left.+\alpha_{\phi}^{2} s_{\phi}^{t} s \phi(r, \psi / w)\right] / c^{2}\left(r, \psi, w, d, d \theta, \phi^{\prime}\right) \\
& \begin{aligned}
c\left(r, \psi, \omega, d_{r}, d_{\theta}, d \phi^{\prime}=\right. & {\left[d_{r}^{2} s_{r}^{t} s_{r}+d_{\theta}^{2} s_{\theta}^{t} s_{\theta}\right.} \\
& \left.+d_{\phi}^{2} s_{\phi}^{t}\right]^{\frac{1}{2}}
\end{aligned} \\
& c_{r}(r, \psi, \omega)=\left[s_{r}^{t}(r, \psi, \omega) s_{r}(r, \psi, \omega)\right]_{\frac{1}{2}}^{\frac{1}{2}}
\end{aligned}
$$

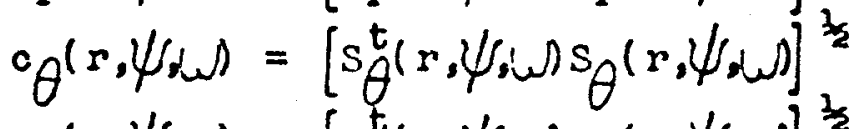

$$
\begin{aligned}
& c_{\phi}\left(r, \psi_{d}\right)=\left[s_{\phi}^{t}\left(r, \psi_{s}, \omega\right) s_{\phi}\left(r, \psi_{,}, \omega\right)\right]^{\frac{1}{2}} \\
& \mathrm{H}_{r}\left(r, \psi_{,}, \omega\right)=\mathrm{s}_{r}\left(r, \psi_{d,}\right) / \mathrm{c}_{r}\left(r, \psi_{d}\right)
\end{aligned}
$$

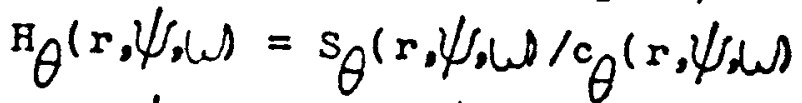

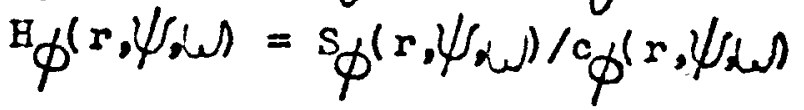




$$
\begin{aligned}
& c\left(r, \psi, \omega, d_{r}, d_{\theta}, d \phi\right)=\int d_{r}^{2} c_{r}^{2}(r, \psi, \omega) \\
& +d_{\theta}^{2} c_{\theta}^{2}\left(r, \psi_{0}(\omega)+\alpha_{\phi}^{2} c_{\phi}^{2}\left(r, \psi_{0}(\omega)\right]^{\frac{1}{2}}\right. \\
& d_{r}^{\prime}=d_{r} c_{r}(r, \psi, \omega) / c\left(r, \psi, \omega, d_{r}, d_{\theta}, d_{\phi}\right) \\
& \mathrm{d}_{\theta}^{\prime}=\mathrm{d}_{\theta} \mathrm{c}_{\theta}\left(r, \psi_{,}\right) / \mathrm{c}\left(r, \psi, \omega, \mathrm{d}_{r}, \mathrm{~d}_{\theta}, \mathrm{d}_{\phi}\right) \\
& \mathrm{d}_{\phi}^{\prime}=\dot{a}_{\phi} \phi_{\phi}(r, \psi, \omega) / \mathrm{c}\left(r, \psi, \omega, \mathrm{d}_{r}, \mathrm{~d}_{\theta}, \mathrm{d}_{\phi}\right) \\
& \left(d_{r}^{\prime}\right)+\left(d_{\theta}^{\prime}\right)^{2}+\left(d_{\phi}^{\prime}\right)^{2}=1 \\
& H\left(r, \psi, \omega, d_{r}, d_{\theta}, d_{\phi}\right)=d_{r}^{\prime} H_{r}(r, \psi, \omega) \\
& +d^{\prime} \theta^{H} \theta^{(r, \psi}(\omega)+d_{\phi}^{\prime} \phi^{\left(r, \psi_{\alpha}\right)} \\
& b\left(r, \psi_{,}^{\prime}, d_{r}, d \theta, d \phi\right)=d_{r}^{\prime} H_{r}^{t}(r, \psi, \omega) v \\
& +d_{\theta}^{\prime}{ }_{\theta}^{t}(r, \psi, \omega) v+\phi_{\phi}^{H} \phi^{t}\left(r, \psi \psi_{d \omega} v\right.
\end{aligned}
$$




$$
\begin{aligned}
& D^{\prime}=\left[d_{r}^{\prime}, d_{\theta}^{\prime}, d_{\phi}^{\prime}\right]^{t} \\
& B=\left[\begin{array}{l}
b_{r} \\
b_{\theta} \\
b_{\phi}
\end{array}\right]=\left[\begin{array}{l}
H_{x}^{t}(r, \psi, \omega) v \\
H_{\theta}^{t}(r, \psi, \omega) v \\
H_{\phi}^{t}(r, \psi, \omega) v
\end{array}\right] \\
& D_{m}^{\prime}=B /|B|
\end{aligned}
$$

The maximum output for an assumed location $(x, \psi, w)$ is again

$$
b_{m}(r, F, \omega)=D_{m}^{\prime} \cdot B=|B|
$$

In the continuous case the approximate dipole location was determined by finding the set of location coordinates which maximized $b_{m}$ in eq. II-38. Similarly, In the discrete case the location is determined by finding the set of coordinates which maximizes $b_{m}$ in eq. II -65 .

To determine the magnitudes of the dipole components, equations II-39 through II -43 may be revised for the discrete case as follows:

$$
\begin{aligned}
b_{r}\left(\bar{r}_{0}\right)= & H_{r}^{t}\left(\bar{r}_{0}\right)\left[d_{r} S_{r}\left(\bar{r}_{0}\right)+d_{\theta} S_{\theta}\left(\bar{r}_{0}\right)\right. \\
& \left.+d_{\phi} S_{\phi}\left(\bar{r}_{0}\right)+r\right] \\
b_{r}\left(\bar{r}_{0}\right)= & d_{r}{ }_{r}^{t}\left(\bar{r}_{0}\right) S_{r}\left(\bar{r}_{0}\right)+H_{r}^{t}\left(\bar{r}_{0}\right) H \\
d_{r}\left(\bar{r}_{0}\right)= & \frac{b_{r}\left(\bar{r}_{0}\right)}{c_{r}\left(\bar{r}_{0}\right)}-\frac{H_{r}^{t}\left(\bar{r}_{0}\right) \mathbb{H}}{c_{r}\left(\bar{r}_{0}\right)}
\end{aligned}
$$


The estimated component values are

$$
\begin{aligned}
& \hat{a}_{r}=b_{p}\left(\bar{r}_{0}\right) / c_{r}\left(\bar{r}_{0}\right) \\
& \hat{a}_{\theta}=b_{\theta}\left(\bar{r}_{0}\right) / c_{\theta}\left(\bar{r}_{0}\right) \\
& \hat{a}_{\phi}=b_{\phi}\left(\bar{r}_{0}\right) / c_{\phi}\left(\bar{r}_{0}\right)
\end{aligned}
$$

and the error components due to nolse are

$$
\begin{aligned}
& \left(\hat{d}_{r}-d_{r}\right)=H_{r}^{t}\left(\bar{r}_{0}\right) N / c_{r}\left(\bar{r}_{0}\right) \\
& \left(\hat{d}_{\theta}-d_{\theta}\right)=H_{\theta}^{t}\left(\bar{r}_{0}\right) N / c_{\theta}\left(\bar{r}_{0}\right) \\
& \left(\hat{d}_{\phi}-d_{\phi}\right)=H_{\phi}^{t}\left(\bar{r}_{0}\right) N / c_{\phi}\left(\bar{r}_{0}\right)
\end{aligned}
$$
$(I I-70 a)$
$(I I-70 b)$
$(I I-70 c)$ 


\section{E. RELATIVE ACCURACY}

In this section, the matched filter procedure will be compared to the procedure used by Schnelder, which was described in Chapter I. As stated in section II-A, it will be assumed that the electrode potentials are measured with respect to a neutral reference. With this restriction, it will be shown that the estimated dipole location and orientation determined by these two procedures will be the same.

The procedure used by Schneider is to find the set of dipole location, orientation, and magnitude parameters which minimize the quantity (eq. I-5):

$$
\zeta=\left|w_{m}-w_{c}\right|^{2} /\left|w_{m}\right|^{2}
$$

The motched filter procedure determines the dipole location and orientation by finding the set of parameters which maximizes

$$
\gamma=v^{t} \mathrm{H} /|\mathrm{v}|
$$

It will be important to note that minimizing $\zeta$ determines the dipole location, orientation and magnitude, while minimizing $\gamma$ determines only the dipole location and orientation. A further step, defined by eq. II-69, is required to determine the dipole magnitude using the matched filter procedure. 
The vector $W_{m}$ in eq. II-7I is the set of measured electrode potentials; vector $W_{m}$ is therefore equivalent to vector $V$ in eq. II-72. Vector $W_{c}$ is the set of theoretical surface potentials produced by a dipole with arbitrary location, orientation, and magnitude. This vector is equivalent to the vector $S$, as defined in eq. II $-44 \mathrm{~b}$. Therefore, using eq. II $-44 \mathrm{~b}$,

$$
w_{c}=s(r, \psi, \omega, \alpha, \beta, d) \text {, }
$$

or

$$
w_{c}=d s_{d}(r, \psi, \omega, \alpha, \beta) \text {, }
$$

or, using eq. II $-47 \mathrm{~b}$,

$$
w_{c}=d c(r, \psi, \omega, \alpha, \beta) H(r, \psi, \omega, \alpha, \beta) \text {. }
$$

In this analysis, it will be convenient to use

a geometrical interpretation of these quantities. Vectors $V$ (or $W_{m}$ ) and $W_{c}$ may be thought of as points in an $m$ dimensionsl space, where $m$ is the number of electrodes. The unit vector $\mathrm{H}$ will be considered to be the direction of a line passing through the origin. Eq. II-75 then indicates that the point $W_{c}$ lies on this line at a distance de from the origin.

By substituting $V$ for $W_{m}$ and eq. II- 75 for $W_{c}$, eq. II-7I becomes

$$
\zeta=\frac{|\mathrm{v}-\mathrm{dcH}|^{2}}{|\mathrm{v}|^{2}}
$$

Since $V$ does not change during the locating process, $\zeta$ can be minimized by minimizing only the numerator 
of eq. II-76. In addition, since the magnitude of a vector is nonnegative, the squaring operation can also be omitted from the numerator. Minimizing $\zeta$ is therefore equivalent to minimizing the quantity

$$
\lambda(r, \psi, \omega, \alpha, \beta, d)=|v-d c(r, \psi, \omega, \alpha, \beta) H(r, \psi, \omega, \alpha, \beta)|(I I-77)
$$

which is the distance from point $V$ to a point, $W_{c}$, which lies on the line defined by $H$.

Let $\mathrm{V}$ be expressed as the sum of two components: $\mathrm{V}_{\mathrm{h}}$, which lies in the direction of $\mathrm{H}$; and $\mathrm{V}_{\mathrm{p}}$, which is orthogonal to H. Thus,

$$
\mathrm{v}=\mathrm{v}_{\mathrm{h}}+\mathrm{v}_{\mathrm{p}}
$$

Since $H$ is anit vector, the component $V_{h}$ is

$$
v_{h}=\left(v^{t}\right)_{H}
$$

and

$$
\mathrm{v}_{\mathrm{p}}=\mathrm{V}-\left(\mathrm{V}^{\left.\mathrm{t}_{\mathrm{H}}\right) \mathrm{H}}\right.
$$

Substituting these equetions into eq. II-77,

$$
\lambda=\left|\mathrm{v}_{\mathrm{p}}+\left(\mathrm{v}^{\mathrm{t}_{\mathrm{H}}}-\mathrm{dc}\right) \mathrm{H}\right|
$$

which, using the generalized Pythagorean theorem, is equal to

$$
\lambda=\left[\left|v_{p}\right|^{2}+\left|\left(v^{t_{H}}-d c\right) H\right|^{2}\right]^{\frac{1}{2}},
$$

or

$$
\lambda=\left[\left|v_{p}\right|^{2}+\left(v^{t_{H}}-d c\right)^{2}\right]^{\frac{1}{2}} \text {. }
$$

It is easily seen that this quantity is minimized with respect to d if

$$
\begin{aligned}
& \mathrm{dc}=\mathrm{v}^{t_{\mathrm{H}}}, \text { or } \\
& \mathrm{d}=\mathrm{v}^{t_{\mathrm{H} / \mathrm{c}}} .
\end{aligned}
$$


Substituting this result back into equation II-77, the minimum value of $\lambda$ with respect to $d$ is

$$
\lambda_{\mathrm{d}}=\mid \mathrm{v}-\left(\mathrm{v}^{\left.\mathrm{t}_{\mathrm{H}}\right) \mathrm{H}} \mid\right. \text {. }
$$

This equation may be expanded to give

or

$$
\lambda_{\mathrm{d}}=\left[|\mathrm{v}|^{2}-2\left(\mathrm{v}_{\mathrm{H}}\right)\left(\mathrm{v}^{t_{H}}\right)+\left(\mathrm{v}^{t_{H}}\right)^{2}\right]^{\frac{1}{2}}
$$

$$
\lambda_{\mathrm{d}}=\left[|\mathrm{v}|^{2}-\left(\mathrm{v}^{t_{\mathrm{H}}}\right)^{2}\right]^{\frac{1}{2}}
$$

Substituting eq. II -72 ,

$$
\lambda_{\mathrm{d}}=\left[|\mathrm{v}|^{2}-\gamma^{2}|\mathrm{v}|^{2}\right]^{\frac{1}{2}}
$$

Finally, since eq. II -77 indicates that

$$
\zeta=\lambda^{2} /|v|^{2}
$$

the minimum of $\zeta$ with respect to $d$ is

$$
\zeta_{\mathrm{d}}=\lambda_{\mathrm{d}}^{2} /|\mathrm{v}|^{2}
$$

or, by eq. II -89 ,

$$
\zeta_{\mathrm{d}}=1-\gamma^{2} \text {. }
$$

Since the magnitude of $\gamma$ cannot be greater than one, it is obvious from eq. II-92 that $\zeta$ will reach its minimum value when $\gamma$ is a maximum. The dipole location and orientation determined using the matched filter will therefore be the same as the location and orientation determined using Schneider's procedure. 


\section{SIMULATIONS}

Tests bave been performed to verify that a two dimensional matched filter can be used to indicate the location of a current dipole, and to investigate the performance of this locating procodure under various conditions. For these tests, computer programs were written which generate simulated surface potential data and then perform the filtering operstion on this data. Filter outputs are ovaluated for different dipole orientetions as a function of the position of the filter dipole (that is, a dipole to which the filter response function is matched).

One purpose of these tests is to determine the resolution of the metched filter with respect to position. It is desirable that the matched filter output decrease very rapidiy as the filter dipole moves away from the actual dipole. Such performance would be advantageous for two reasons. First, if more than one source were present, a plot of the filter output would then show a distinct spike indicating the position of each source. Second, a narrow peak should make it possible to determine the source position with greater precision when noise is present.

Another purpose of these tests is to investigate 
the effects of some practical problems rolated to the implementation of the matched filter. Matters which have been simulated include the effect on filter resolution of the use of discrete surface potential samples rather than continuous data, changing of the number of electrodes, and the omission of electrodes on a portion of the surface. These problems will be described more fully in the discussions of the individual tests.

In all of the tests to be described, the theoretical surface potentials were calculated using the single sphere model of the human head. The signal source is a single current dipole oriented in either a radial or tangential direction. The simulation programs calculate the resulting response of one or more of the component filters whose impulso responses are defined in eq. II-29. Multiple sources, noise sources, and possible distortions due to inaccuracies of the model are not considered in these simulations.

It has been shown by schneider ${ }^{19}$ that the surface potentials predicted by the single sphere model for dipoles near the surface are substantially inaccurate. Therefore, primary emphasis in these simulations has been. placed on dipoles located between the center and $70 \%$ of the radius of the sphere.

To calculate the surface potentials, the dipolo magnitude and certain physical parameters of the model 
must be specified. For the single sphere model, the two required parameters are the radius of the sphere and its conductivity (see appendix A). However, from equations A19 through A21 of appendix A, it can be seen that the only result of a change in the values of these parameters, or of the dipole magnitude, is to scale the potential at all points on the surface by a constant factor. As a result, the shape of the response curves of the component filters may be determined using arbitrary values for these parameters; the actual values of the component filter outputs may then be determined, if desired, by applying a scale factor derived from the actual values of these parameters.

For purposes of these simulations, the factor $\left(\mathrm{P}_{1} / 4 \pi \sigma^{\mathrm{R}}\right)$ of equations $\mathrm{A} 19$ through $\mathrm{A} 21$ is adjusted so that the integral of the square of the potential over the surface is unity. This selection produces two advantages. First, it causes the filter output to equal unity when the actual dipole and filter dipole are identical, which facilitates comparison of different curves. Second, as can be seen from equations II-28 and II-29, the constant of proportionality, $c$, becomes unity, so that the surface potential due to an actual dipole is now identical with the implise response of a filter dipole having the same location and orientation. As a result, the actual dipole and filter dipole may be 
interchanged without affecting the filter output, thus halving the amount of computation required in many of the simulations.

The simulation results are presented in this chapter in the form of graphs. The programs used and the actual data generated are listed and described in appendix B. 


\section{A. SURPACE POTENTIALS}

Before simulating the matched filter, the surface potential functions were colculated for radial and tangential dipoles using the single sphere model.

As shown in oppendix A, if the coordinate system is chosen such that the dipole lies on the $z$ axis, the potential due to the radial component is Independent of $\phi$, while the potential due to a tangential component is a sinusoidal function of $\phi$. Therefore, the surface potentials were calculated and plotted as a function of $\theta$ only.

Plots of surface potential as anction of dipole depth are shown in figures 2 and 3 . An observation which may be made from these figures is that the shape of the curves changes only slightly with changes in dipole depth. Effects of this fact will be discussed more fully in the next section. It will also be noted that the signal tends to become concentrated in a smaller region around the 2 axis as the dipole moves closer to the surface.

Total signal energy" was also calculated for

\footnotetext{
अAs was stated In section II-B, the onergy is defined here as the integral of the squere of the voltage over the surface.
} 


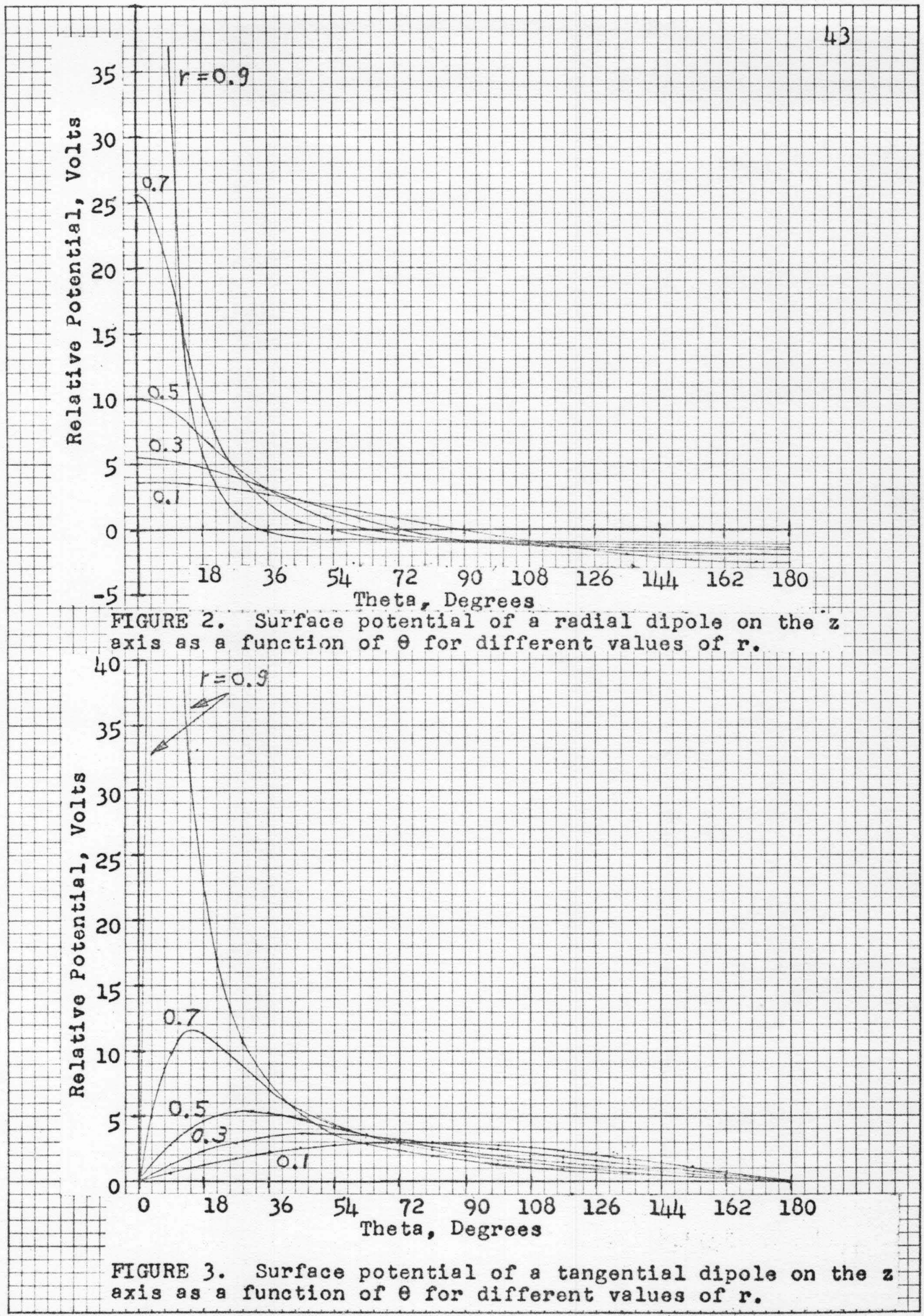


each curve and is listed in table I. It will be noted that the signal energy is much higher for dipoles noar the surface and is slightly higher for radial than for tangential dipoles. 
TABLE I

RELATIVE SIGNAL ENERGIES PRODUCED

BY CURRENT DIPOLES

$\begin{array}{lcc}\text { POSITION } & \text { RADIAL DIPOLE } & \text { TANGENTIAL DIPOLE } \\ 0.1 & 38.3 & 38.2 \\ 0.2 & 40.4 & 39.7 \\ 0.3 & 44.2 & 42.5 \\ 0.4 & 50.6 & 47.1 \\ 0.5 & 61.4 & 54.7 \\ 0.6 & 81.0 & 67.9 \\ 0.7 & 121.3 & 93.9 \\ 0.8 & 228.8 & 159.4 \\ 0.9 & 762.2 & 460.2\end{array}$




\section{B. RESOLUTION TESTS}

To test the sensitivity of the notched filter to changes in the location of the dipole, the filter output was calculated and plotted for several types of mismetch between the filter and the dipole coordinates.

Calculations were made of the filter response as a function of the dipole location parameters $r$ and $\psi$. These calculations were made for both radial and tangential dipoles. In addition, the output of a tangential filter detecting radial dipolo was calculated as a function of $\psi$.

Flgure 4 shows the filter response for variation of $x_{\text {. }}$ Both radial and tangential dipoles lie on the 2 axis at $r=0.6$ with the tangential dipole oriented in the $\overline{\bar{B}}_{\phi}$ direction. The curves for a radial filter detecting a radial dipole and for a tangential filter and dipole are drawn from data polnts spaced $.02 \pi$ radians apart. It will be noted that the filter is more sensitive to $\psi$ variation for a tangontial dipolo. than for a radial one.

The third curve of $\mathrm{fig} .4$ is the response of a radial filter to a tangential dipole for equivalently, as explained in the introduction, the response of a tangential filter to a radial dipole). 


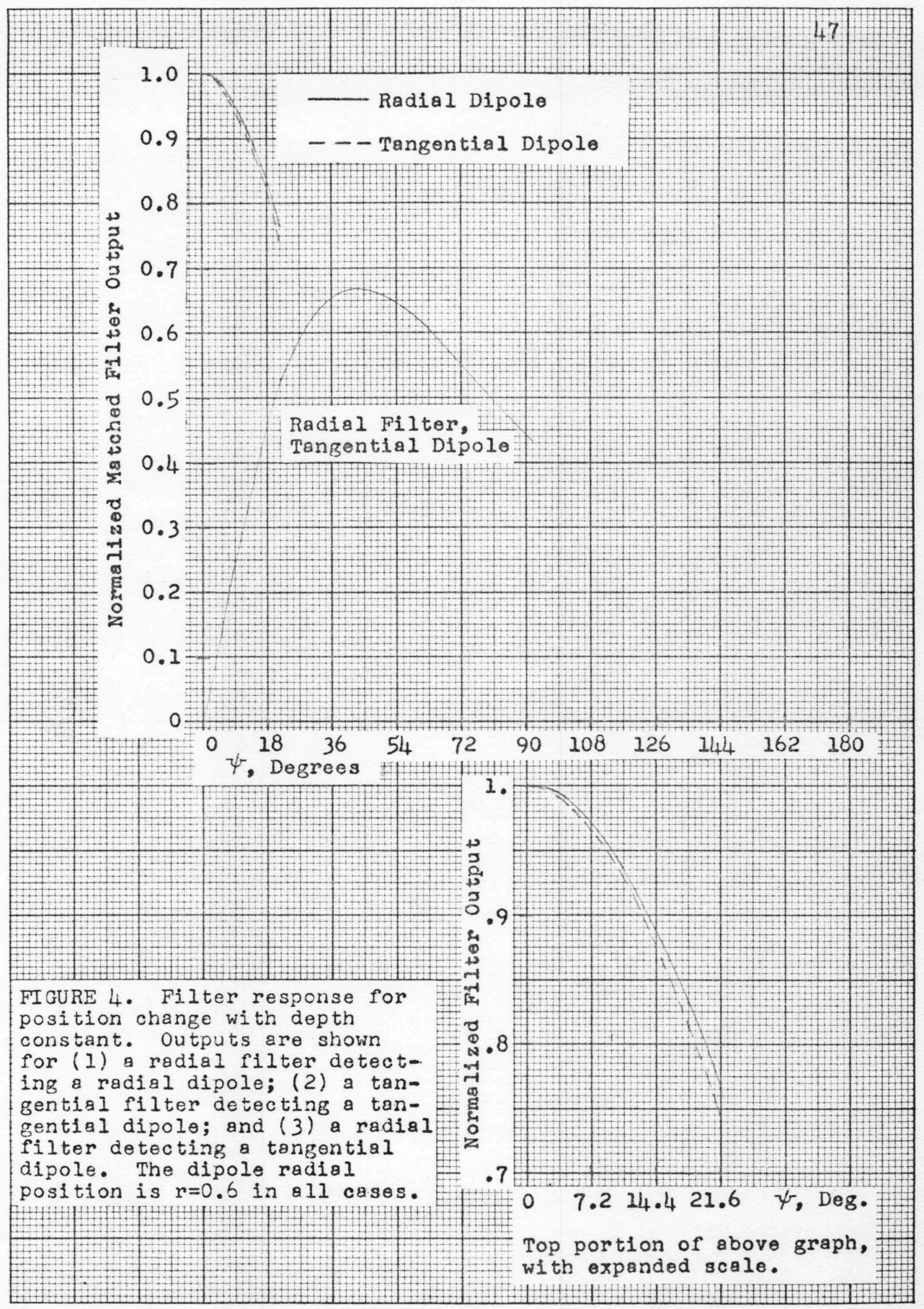


Thl s curve $1 \mathrm{~s}$ drawn from data points spaced .05 radians apart. The maximum value is approximately $\gamma=0.67$ at $\psi=0.25 \pi$. When the filter and dipole positions are the same $\left(x_{r}=0\right)$, the dipole potential is orthogonal to the filter response function and the output is zero, as expected.

Figure 5 shows the response of the filter to changes in $r$ for actual dipoles located at $r=.1, .3$, $.5, .7, .8$, and .9 . Comparison with fig. 4 shows that: the filter is much less sensitive to variation in $r$ than to variation in $\%$. It can also be seen that in this case the filter is more selective for radial than for tangential dipoles.

This lack of sensitivity to $r$ is not surprising since, from figures 2 and 3, the surface potentiol itself changes only slowly with changes in $r$. 


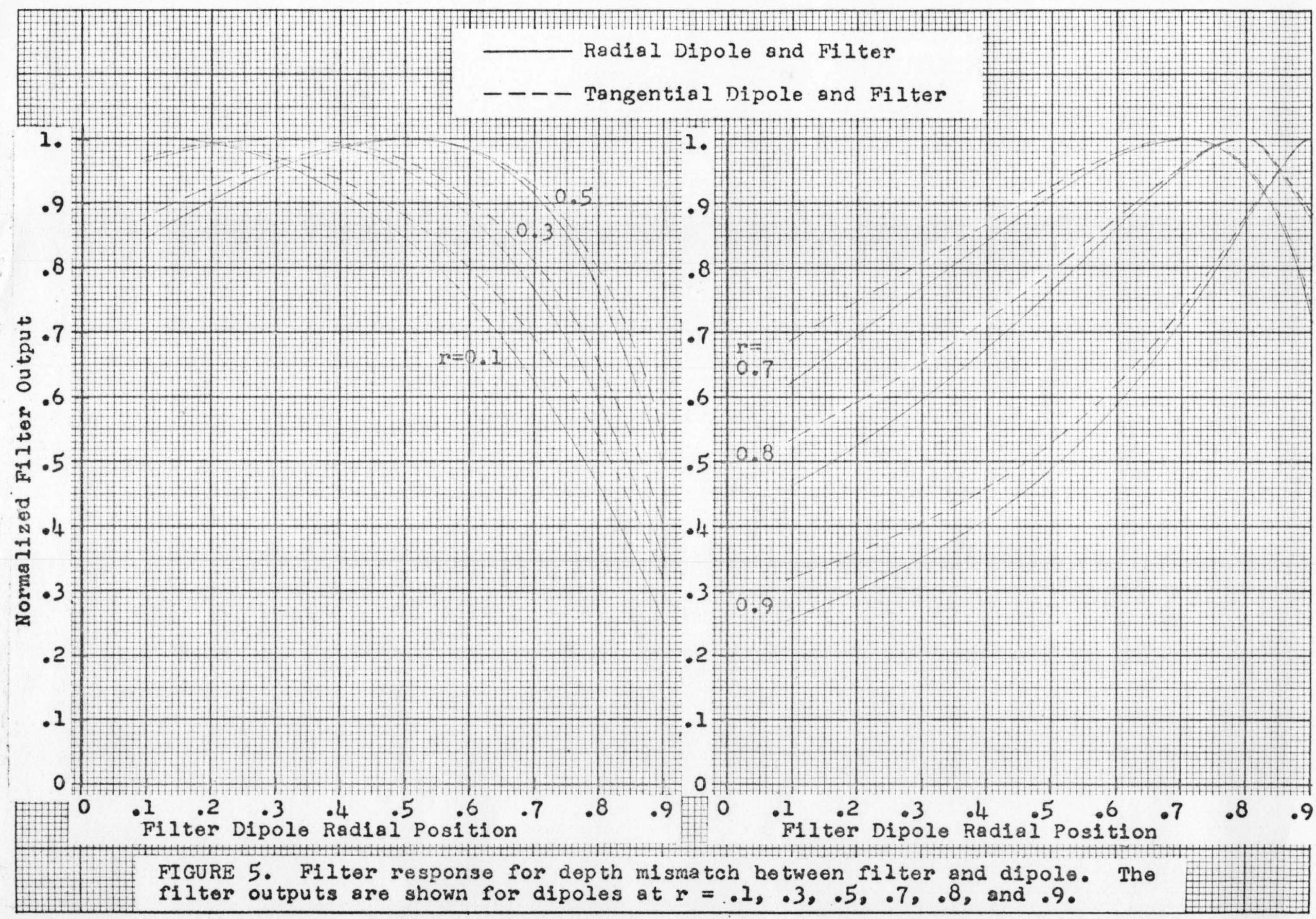




\section{EFPECTS OF NUMEER OP ELECTRODES}

In practice, the surface potential is known only at the electrode locations and not at every point on the surface. It is therefore of interest to deteralne how the selectivity of the loceting procodure is affected by varlations in the number of electrodes.

To simulate operation with a finite number of electrodes, a computer progran was written to evaluato the filter outputs given by eq. II-53 (Seo appendix B for the progran and printouts). A series of runs wore made for depth mismatch using from 8 to 97 eloctrodes on each hemisphere with the electrode positions determined by an algorithm described in appendix $B$. The actual dipole used in each case is a radial or tangential dipole at $\mathbf{r}=0.5$. Results are plotted in figures 6 and 7 for 8 to 66 electrodes per hemisphere. Comparing rigures 6 and 7 to fig. 5 , it can bo seen that the fllter response shapes approsch those of the continuous case as the number of electrodes increases. Using $B$ electrodes por homisphero, the filter shows much less sengitivity to changes in $r$ near the peak of tho curve. Increasing the numbor of eiectrodes to 21 per houlsphere causes the filter response to approanb the continuous case over the range from $r=0.4$ to $r=0.6$. Funther increseg in the numbe or tactrodes wider the 


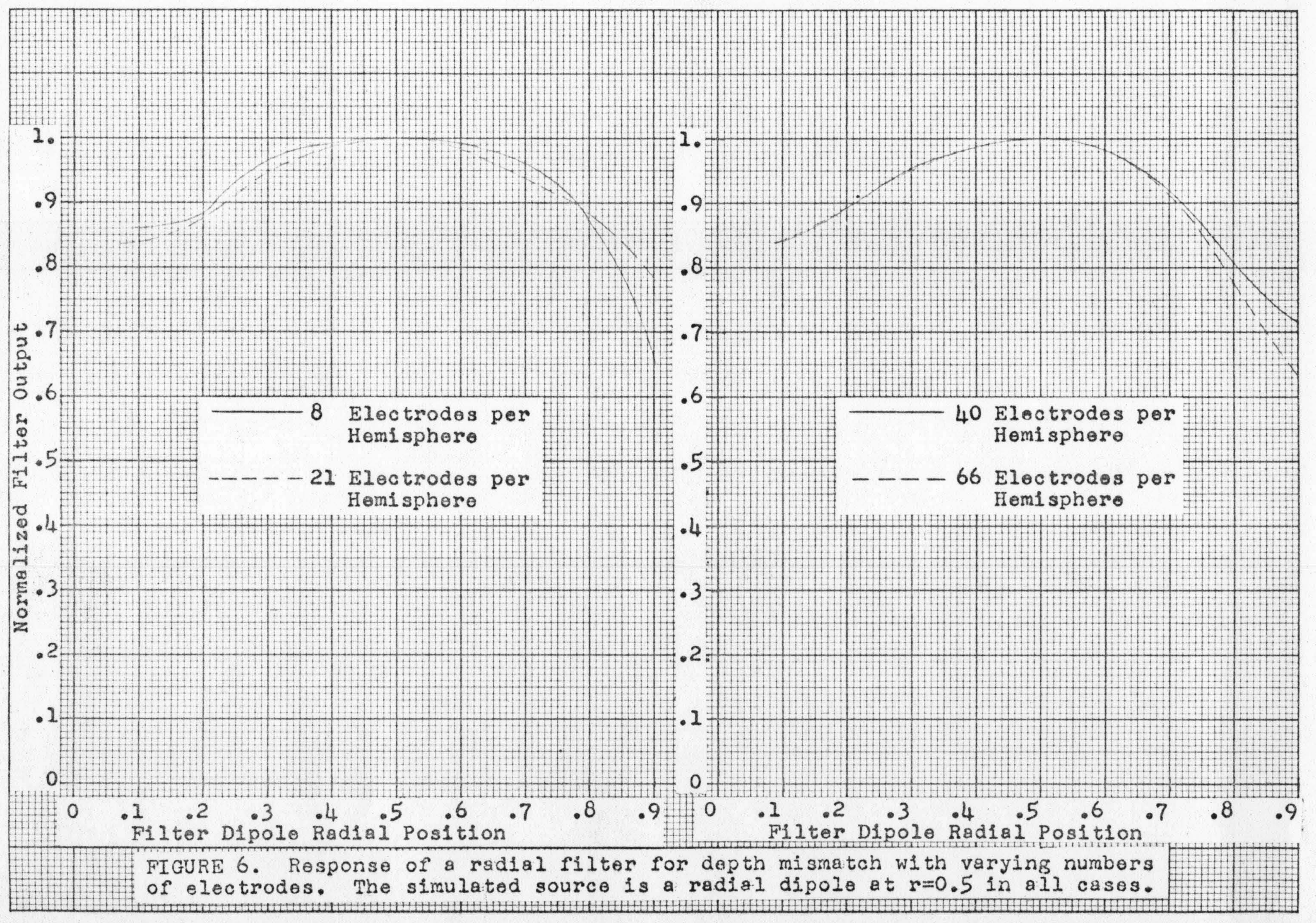




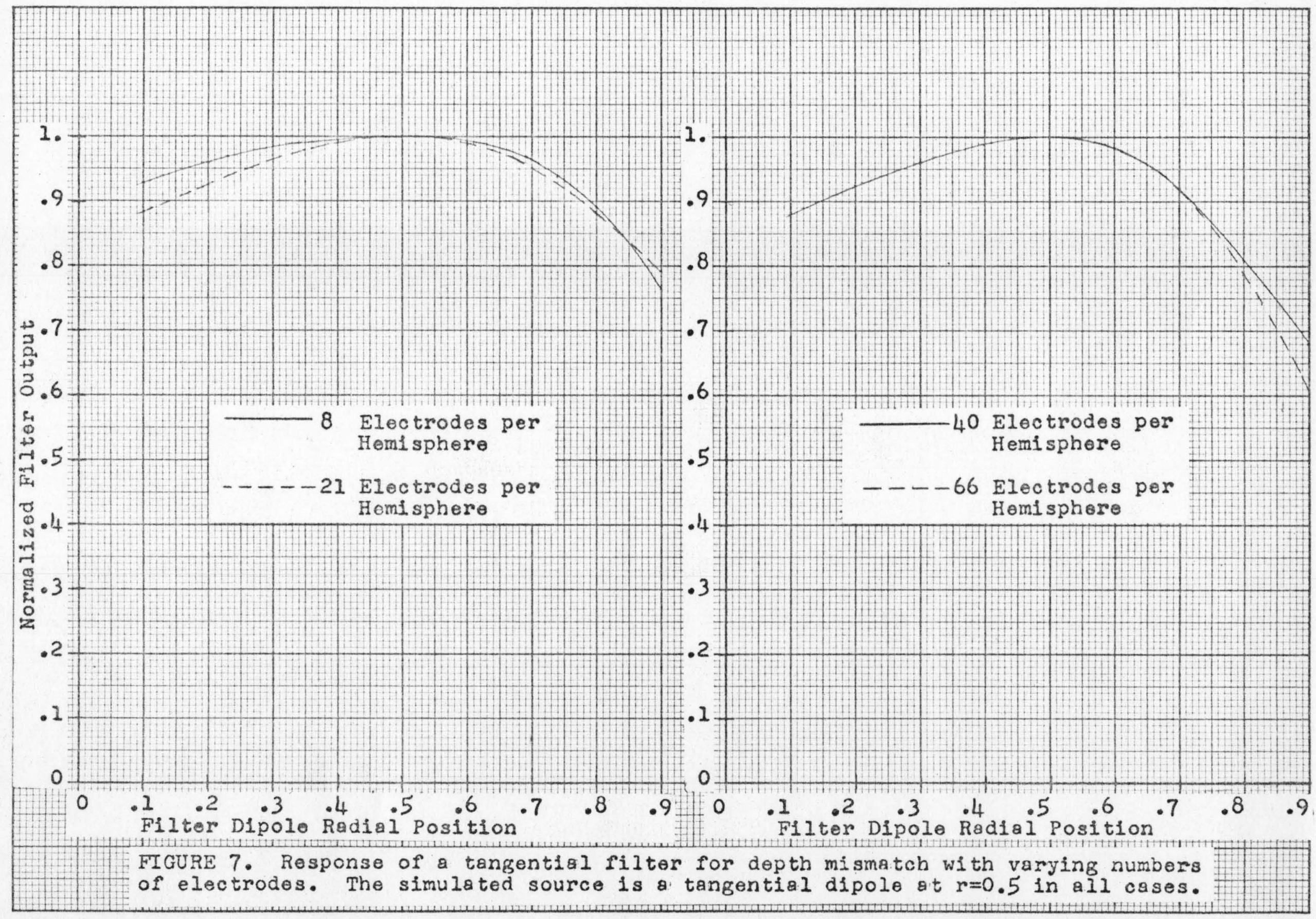


range over which the filter output for the discrete case agrees with the continuous case.

Besed on these results, some observations may be made regarding the number of olectrodes per hemisphere to be used.

(1) Due to the flatness of the curve, a substantial loss of accuracy may be expected in the case of 8 electrodes when the effect of noise is considered.

(2) Because the filter output with 21 electrodes nearly matches the continuous case in the region near the peak of the curve, it is not clear whether the use of more than 21 electrodes per hemisphero will produce a significant increase in accuracy.

A detalled analysis of the effects of nolse on the results will be required before the latter question can be resolved.

Both observations must be qualified in that they are based on changes in filter sensitivity to varlation in $r$ only. Changes in sensitivity to variations In $\psi$ and $\omega$ should bo inveatigated also. 


\section{EFFECT OF SURFACE TRUNCATION}

The simulations described in the previous section assumed that the electrode locations were spread evenly over the entire spherical surface. In reality. the possible locations are limited by the shape of the hood to approximately one hemisphere. To invostigate the effent of this limitation, the filter outputs described In section $C$ were recalculated using only the electrode locations on the hemisphere for which 2 is greater than zero. These results are shown in figures 8 and 9. The curves of flgures 8 and 9 show very 11 ttlo change from the curves of figures 6 and 7 . One reason for the lack of change is the fact that the dipole is near the $z$ axis. From figures 2 and 3, it can be seen that the surface potential is smoll on the hemlsphere for which z is negative (1. … $\theta>\pi / 2)$. Thereforo, very IItte of the signal energy is lost by neglecting the contribution of this hemisphere. While simulations were not mede for other dipole locations, it is expected that filter selectivity using one hemlsphere will decrease as the dipolo moves closer to the unused hemisphere and more signal energy is lost due to surface truncation. 


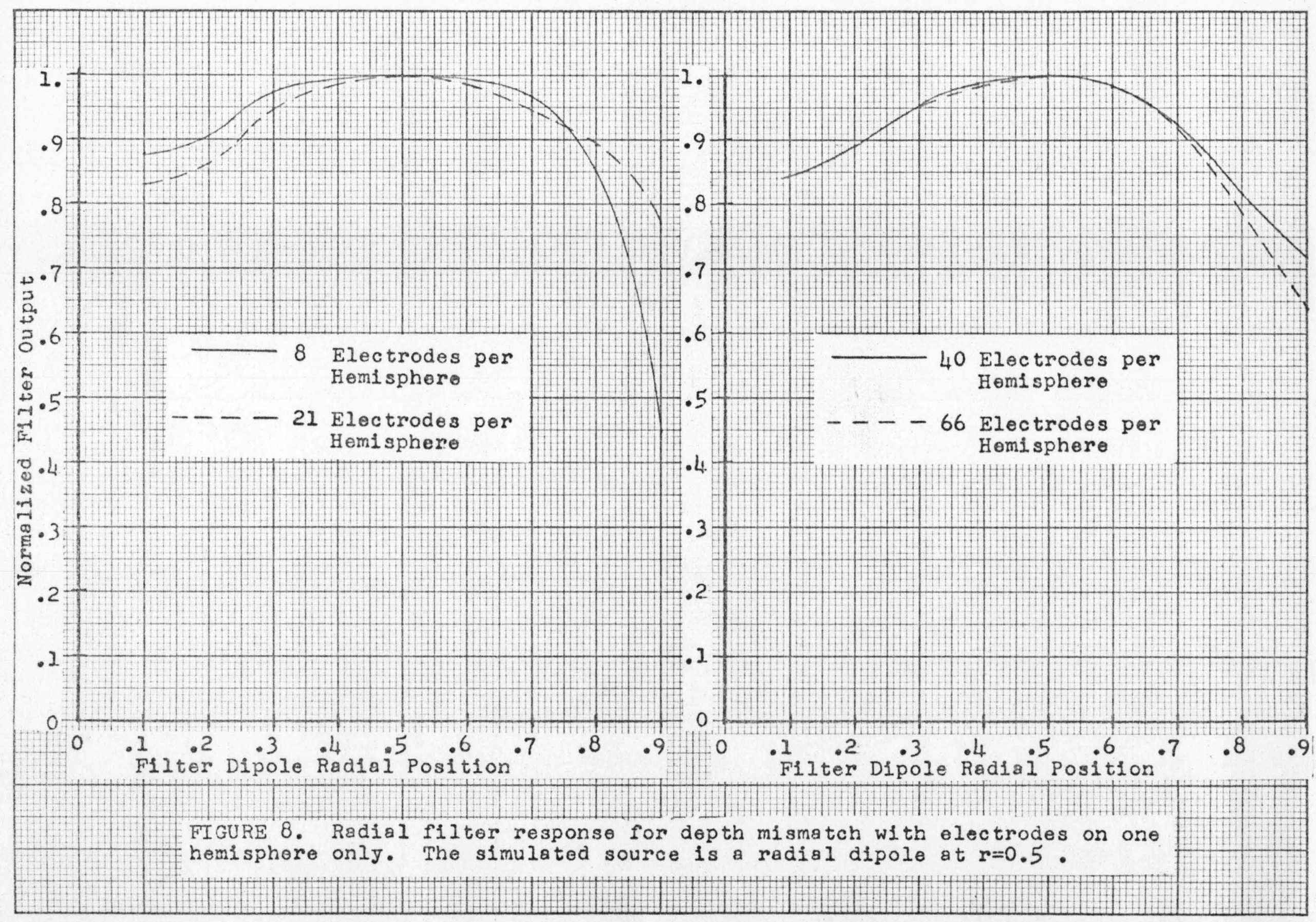




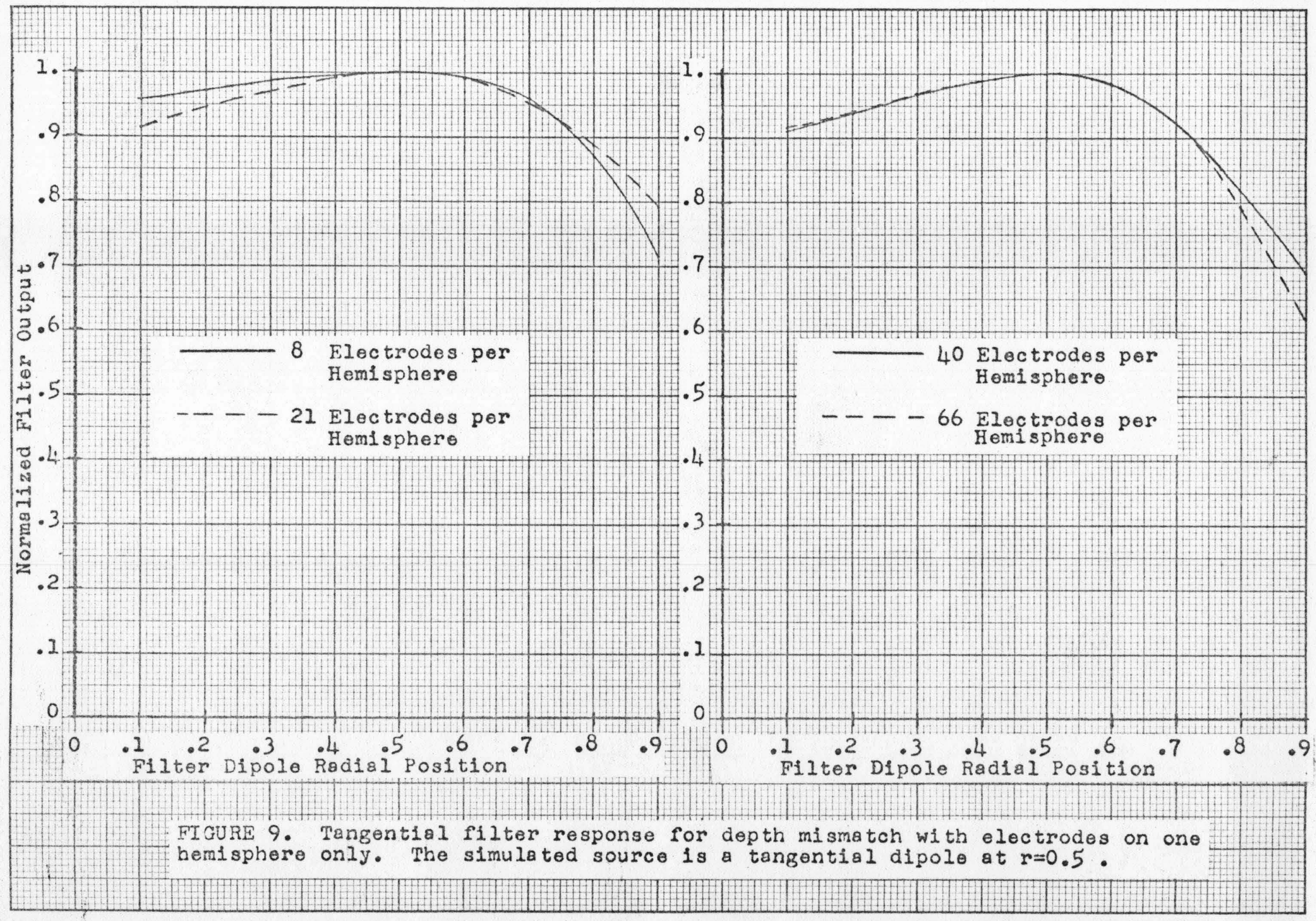




\section{E. CONCLUSIONS}

In summary, the following conclusions may be reached regarding the matched filter locating procedure as a result of the computer simulations which have been performed:

(1) The matched filter detection process exhibits more sensitivity to changes in $\psi$ and $\omega$ than to changes in $r$. Therefore, it is expected thet the depth of a dipole source cannot be determined to the same degree of precision as its lateral position.

(2) Filter selectivity tends to sncrease as the number of electrodes increases. In particular, for the cases simulated, it was found that use of 8 or fewer electrodes por hemisphere produces a substantial loss of selectivity; while the use of more than 21 electrodes per hemlsphere produces only slight increases in solectivity.

(3) Inability to measure potentials over a portion of the surface does not necessarily result in a large decrease in selectlvity. For the cases simulated, the potentials omitted were of low magnitude; and under these conditions the effect of their omission 
on the filter selectivity was sight.

In the course of these simulations, some areas which merlt further investigation were identified. These areas ore as follows:

(1) In the simulations for a finite number of electrodes, the effects of modifications on filter sensitivity to changes in $r$ were observed. Fur ther tests should be made to determine the effects of these modifications on the sensitivity to $\psi$ and $w$ variation.

(2) The electrode positions used in these simulations were chosen to provide an even distribution over the surface area. However, Improvements in selectlvity for a given number of electrodes may be possible by modifying the electrode positions. Investigations to determine an optimum electrode pattern should be conducted.

(3) Since the single sphere model is known to be inaccurate for dipoles near the surface, the results of these simulations should be verified using the three spheres model.

(4) While the obtainable accuracy of the locating procedure is obviously related to the selectlvity of the filter, a quantitative statement of their relation- 
ship will require an analygis of the effects of nolse on the filter output. Such an analysis has been started; the incomplete results ore shown in appendix $C$. 


\section{SUAMARY}

A now technique has been presented for determining the location, orientation, and magnitude of a dipole current source in a volume conductor by measuring the surface potential at a finite number of points. This technique is based on the application of the matched filter concopt of communications thoory to a spherical surfaco. In addition, it has been shown that the amount of computation required can be greatly reduced by resolving an arbitrary dipole into threo components with fixed orientation and considering each component separately. Computer simulations have also been performed to demonstrate the operstion of this technique.

It was shown in section II-E that the matched filter should produce approximately the same result as other opproaches which have been used previously; Improved accuracy is therefore not an advantage of this technique. The matched filter method does, however, offer some advantages which will now be discussed.

The provious locating method, as used by Schneider, was based on an iterative search for the set of parameters providing a best fit to the observed data. An iterative procedure requires the selection of an inftial set of values 
for the unknown quantities and the specification of some criterion to determine when the search is to bo terminated. As a result, fallure to obtain convergence mey occur elther because the source bolng sought does not exist or because the search was started or terminated lmproperly.

Since the matched filter mothod doos not require the use of an iterative procedure, this limitation does not apply to 1t. If no peak is observed in the filter output, it can then be stated conclusively that no source exists which can be modeled as a single current dipole. The only requirement is that the filter output be calculated for points close enough together that the peak cannot fall between output samples and be missed. This spacing can be determined from curves, such as those prosented in chapter III, which show the width of the filter output peak.

The matched filter procedure also has other potential advantages. One of these advantages is the posslbility of porforming a theoretical analysis of the avaliable accuracj, given the signal to noise ratio at the electrodes. Another potential advantage is the possiblitty of decreasing the procossing time by increasing the amount of morory used. The beginning of an orror analysis is presented in appendix $\mathrm{C}$; the second advantage is discussed more fully in the next soction. 


\section{RECOMMENDATIONS FOR FUTURE WORK}

The matchod filter algorithm has been presented, and its operation has been demonstrated using simulated data. The next step appoars to be a series of tosts to determine how well this algorlthm will perform using real input data.

Such tests should be used not only to verify that this algorithm works correctly, but also to characterize the nolse that is present in actual data. Characteristics of importance includo the statistical paramoters of the noise a a function of surface position and the correlation of nolse samples at different points on the surface. This information will be useful in the theoretical analgsis of available accuracy and will also permit simulations to be performed which more closely resemble the real world. Since, as stated previously, it has been reported in the ilterature that the single sphere model introduces substantial inaccuracy, it is suggested that these and all subsequent studies be performed using the three spheres model.

Assuming the initial tests produce satisfactory results, three areas related to tho matched filter algorithm havo been identifled which doserve further study. 
These areas will now be discussed.

One problem with the matched filter locating procedure, as woll a schnelder's procedure, is that the amount of computation required to caloulate the theoretical potentials increases substantially as tho complexity of the model increases. Therefore, for the three spheres model in particular, it may be advantageous to decrease the processing time by using a lookup table to provide the theoretical surface potentials for a large number of dipole locations within the rolume conductor. For the matched filter procedure, such a tablo would contain for each location the filter response vectors for the three component dipoles. By way of example, if the coordinates $r, \psi$, and $w$ are divided into ten segments each. (produoing 1000 dipole locations), and if 21 electrodos are used, a total of 63000 words of memory would be required to store the three response vectors for each location. Th1s amount of storage is within the capacity of many minicomputers, so such an approach is definitely feasible. The primery questions to be answered in future Investigations aro the number of points to be used and thelr locations. For example, the slmulations presented In chapter III showed that the filter procedure has low sensitivity to change in dipole depth. Therofore, the $r$ coordinate would probably be divided into fewer segments 
than were assumed in the above example. In addition, It is expected that the number of segments for the $\psi$ and $\omega$ coordinstes would be decreased for the locations noar the center. The number and locations of points will be a compromise between the requirements, first, that the points bo sufficiently close together that at lesst one calculated output will be near the peak of the filter output, and second, thet they bo chosen as far apart as possible so that the amount of memory and computation required will be minimized.

A second area to be investigated is the possibility thet the matched filter can be used even if more than one dipole source is present. Because the three component filters perform linear operations on the data, the filter output for two dipoles will be the sum of the outputs which would be produced by the dipoles ind1vidually. Two peaks should therefore be observed in the fliter output, one corresponding to the location of each dipole. The primary question to be answered in this regerd is whother the two peaks at the dipole locations can be distingulshed from other peaks such as those observed in section III-B caused by the interaction of a radial filter and a tangential dipole.

The third question to be studied is the effect that different sensing techniques have on the obtainable accuracy. Three types of connections are commonly used 
In studies of the EEG. 24 First, the potentials may be messured differentialiy between polrs of electrodes. Second, the potentials may be measured with the average of all electrode potentials used as a reference. Third, a neutral point, such as an ear clip, may bo used as a referenco.

The matched filter procodure implicitly assumes the use of the neutral reference, since the surface potentisls due to the three component dipoles will not necessarily be or thogonal if el ther of the first two types of connections is used. A question which therefore arises is whether this restriction bas any offect on the obtainable accuracy. An advantage of the third type is that the nuraber of independent data samples is equal to the number of electrodes, whereas the maximum number of independent samples is one less than the number of electrodes for the othor two schemes. Prollminary tests using the simulation programs were made to compare the second and third types of oonnections (see appendix B, figures B14 and B15, for the applicable printouts). These results indicate that the use of a noutral reference may provide an fmprovement in performance. Howevor, because these slmulations do not include noise effects, it cannot yet be stated with certalnty which of the three coninection methods is best. 


\section{APPENDICES}


APPENDIX A. SURFACE POTENTIAL EQUATIONS

In this appendix the equations for the surface potnetial produced by a single current dipole in a spherical volume conductor will be presented for both the single sphere and the three concentric models. It will then be shown that the surface potentials produced be the three components of the dipole are mutualig or thogonal.

The single sphere potential equations are available in the literature; the following set of equations for a rectangular coordinate system are taken from ref. 6.

With the origin at the center of the sphere,

let

$$
\begin{aligned}
& X, Y, Z \text { be the coordinates of point } A \text { on the } \\
& \text { spherical surface; } \\
& R \text { be the radius of the sphere; } \\
& \sigma \text { be the conductivity of the medium; } \\
& \mathrm{P}_{1}, \mathrm{P}_{2}, \mathrm{P}_{3} \text { be the coordinates of the dipole center; } \\
& P_{4}, P_{5}, P_{6} \text { be the } X, Y \text {, and } Z \text { components, respectively, } \\
& \text { of the dipole moment. }
\end{aligned}
$$

* The notation used here follows that of reference 6 , rather than that of the main body of this thesis. 
Now, define

$$
\begin{array}{ll}
D=\left(P_{1}^{2}+P_{2}^{2}+P_{3}^{2}\right)^{\frac{1}{2}} & A 1 \\
d=D / R & A 2 \\
H_{1}=\left(P_{1} P_{4}+P_{2} P_{5}+P_{3} P_{6}\right) / D & A 3 \\
H_{2}=\left(P_{4} X+P_{5} Y+P_{6} Z\right) / R & A 4 \\
H=\left(P_{1} X+P_{2} Y+P_{3} Z\right) /(D R) & A 5 \\
L=\left(1+d^{2}-2 H d\right)^{\frac{1}{2}} & A 6 \\
S=\frac{2}{L^{3}}+\frac{d-H}{d\left(1-H^{2}\right) L}+\frac{H}{d\left(I-H^{2}\right)} & A 7 \\
T=\frac{2 d}{L^{3}}+\frac{d H-1}{d\left(1-H^{2}\right) L}+\frac{1}{d\left(I-H^{2}\right)} & A 8
\end{array}
$$

The potential at point A due to the dipole is then

$$
\mathrm{V}(\mathrm{A})=\left(\mathrm{SH}_{2}-\mathrm{TH}_{1}\right) /(4 \pi \sigma \mathrm{R})
$$

For later use it will be necessary to convert to a spherical coordinate system. First, the rectangular coordinete system may be defined without loss of generality such that the dipole is located on the positive $z$ axis. This definition implies that $P_{1}$ and $P_{2}$ become zero. Now, equations Al through A9 may be modified to become:

$$
\begin{aligned}
D & =P_{3} \\
d & =P_{3} / R \\
H_{1} & =P_{6} \\
H_{2} & =\left(P_{4} X+P_{5} Y+P_{6} Z\right) / R \\
& =P_{4} \sin \theta \cos \phi+P_{5} \sin \theta \sin \phi+P_{6} \cos \theta \\
H & =Z / R=\cos \theta \\
L & =\left(1+d^{2}-2 d \cos \theta\right)^{\frac{1}{2}}
\end{aligned}
$$




$$
\begin{aligned}
& S=\frac{2}{L^{3}}+\frac{d-\cos \theta}{d L \sin ^{2} \theta}+\frac{\cos \theta}{d \sin ^{2} \theta} \\
& T=\frac{2 d}{L^{3}}+\frac{d \cos \theta-1}{d L \sin ^{2} \theta}+\frac{1}{d \sin ^{2} \theta} \\
& V(A)=\left(S P_{4} \sin \theta \cos \phi+S P_{5} \sin \theta \sin \phi+\right. \\
& \left.\quad S P_{6} \cos \theta-T P_{6}\right) /(4 \pi \sigma R)
\end{aligned}
$$

The $\bar{a}_{X}, \bar{a}_{y}$, and $\bar{a}_{z}$ unit vectors are equivalent to the $\bar{a}, \bar{a}$, and $\bar{a}_{\boldsymbol{r}}$ unit vectors, respectlvelJ, for a point on the $z$ axis. Therefore, since $P_{4}, P_{5}$, and $P_{6}$ are the dipole components in the $\bar{a}_{x}, \overline{\bar{a}}_{y}$, and $\overline{\boldsymbol{a}}_{z}$ directions, respectively, the surface potential contributions from the three components of the dipole are:

$$
\begin{aligned}
& V_{p}(A)=\frac{S \cos \theta-T}{4 \pi \sigma R} P_{6} \\
& V_{\theta}(A)=\frac{\sin \theta}{4 \pi \sigma R}(\cos \phi) P_{4} \\
& V_{\phi \phi}(A)=-\frac{\sin \theta}{4 \pi \sigma R}(\sin \phi) P_{5}
\end{aligned}
$$

The oquations defining trie surfoce potentsal

for tho throe spherea modal are also araliabie in the 11 terature. The set of equations presented here is rrom ref. 19.

The coordinate system for the three spheres model is again defined such that the center of the coordingto system is a the center of the three spheres and the dipole Iles on the positive $z$ axis and is in the inner sphere. The 
brain is modeled as the inner sphere of conductivity 8 , the skull is represented by the intermediate shell of conductivity $\mathrm{kg}$, and the scalp is the outer shell of conductivity fg. The current dipolo has magnitude $p$ with components $p_{r}, p_{\theta}$, and $p_{\phi}$. The dimensions of the model are shown in flg. Al, while typical values of the constants are listed in table Al.

For point $A$ on the surface, with coordinates $(R, \theta, \phi)$, the surface potential contribution from each of the dipole components 13:

$$
\begin{aligned}
& v_{r}(A)=\frac{1}{4 \pi g J R^{2}}\left[\sum_{n=1}^{\infty} \frac{(2 n+1) 3_{k f^{n-1}}}{(n+1) D_{n}} P_{n}^{0}(\cos \theta)\right] p_{r} \quad \text { A22 } \\
& V_{\theta}(A)=\frac{1}{4 \pi g J R^{2}}\left[\sum_{n=1}^{\infty} \frac{(2 n+1)^{3} k r^{n-1}}{n(n+1) D_{n}} P_{n}^{1}(\cos \theta)\right](\cos \phi) p_{r} \quad \text { A23 } \\
& V_{\phi}(A)=\frac{1}{4 \pi g J R^{2}}\left[\sum_{n=1}^{\infty} \frac{(2 n+1) 3_{k f^{n-1}}}{n(n+1) D_{n}} P_{n}^{1}(\cos \theta)\right](\sin \phi) p_{r} \quad A 24
\end{aligned}
$$

where $P_{n}^{0}$ and $P_{n}^{1}$ are the Legendre polynomials, and

$$
\begin{aligned}
D_{n}= & (k n+k+n)(n k / j+n+1) /(n+1) \\
& -(k n+k+n)(1-k / j) c^{2 n+1} \\
& +(1-k)(n+k(n+1) / j) b^{2 n+1} \\
& -n(1-k)(1-k / j)(b / c)^{2 n+1}
\end{aligned}
$$




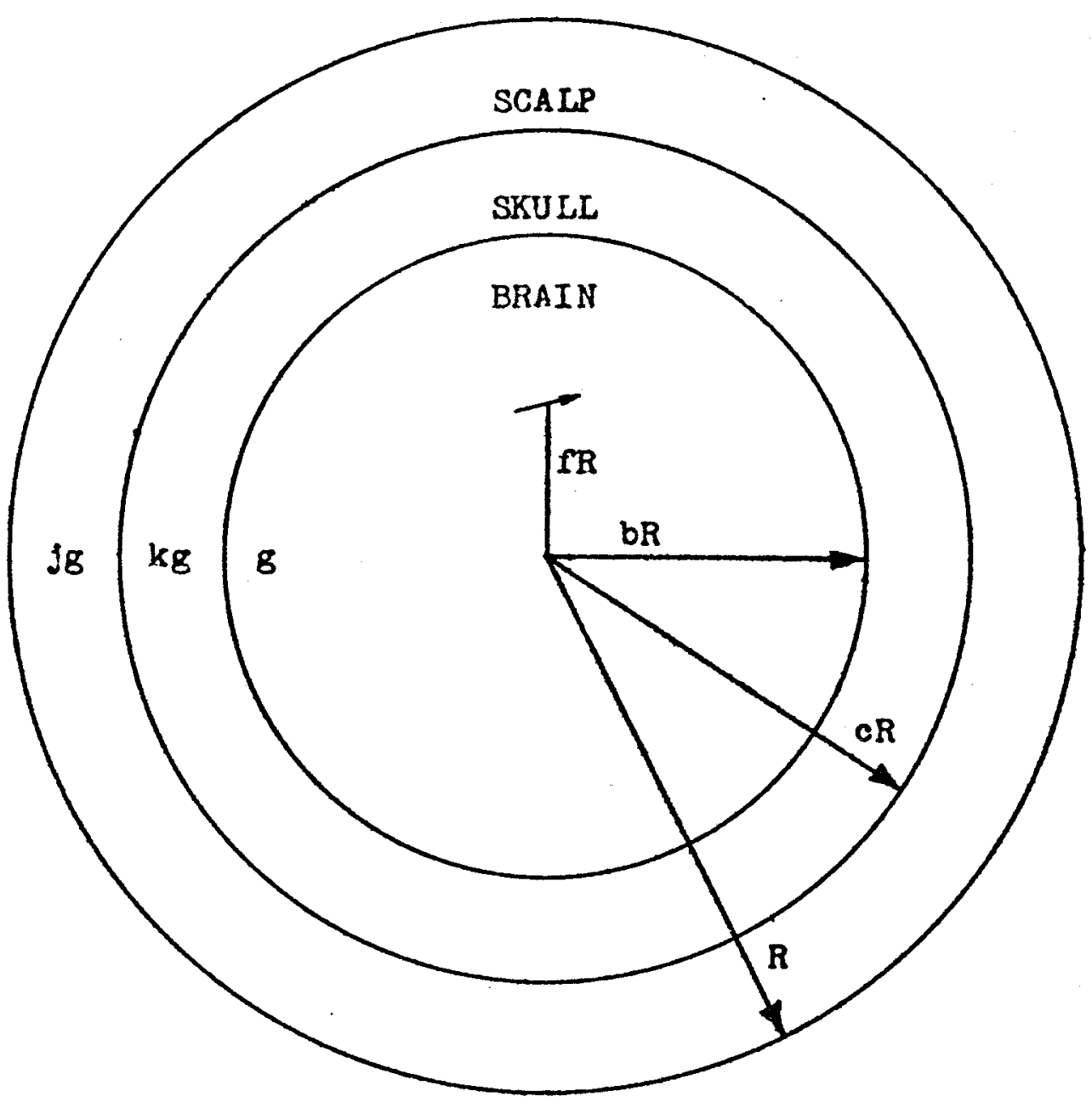

FIGURE Al. The three spheres model of the human head. 
TABLE AI

PARAMETERS OF THE THREE SPHERES MODEL

(From Reference 4)

PARAMETER

$\mathbf{R}$

b

c

8

J

k
VALUE

$9.2 \mathrm{~cm}$

0.87

0.92

0.0045 mho/om

1.0

0.0125 
To prove that the surface potential functions of the three component dipoles are mutualiy or thogonal, note thet for both the single sphere and three spheres models the surface potentials due to the three components are of the forms

$$
\begin{aligned}
V_{r}(A) & =F_{1}(\theta) p_{r} \\
V_{\theta}(A) & =F_{2}(\theta)(\cos \phi) p_{\theta} \\
v_{\phi}(A) & =F_{2}(\theta)(\sin \phi) p_{\phi}
\end{aligned}
$$

where $F_{1}$ and $F_{2}$ are functions which depend on the dimensional and electrical constants of the model and on $\theta$, but not $\phi$. Therefore, defining the inner product of two functions of $\theta$ and $\phi$ as the integral over the entire surface of their product, the inner product of the functions $v_{r}$ and $v_{\theta} 1 s$

$$
\begin{aligned}
\left\langle v_{r} \mid v_{\theta}\right\rangle & =\int_{0}^{2 \pi} \int_{0}^{\pi} v_{r}(R, \theta, \phi) v_{\theta}(R, \theta, \phi) R^{2} \sin \theta d \theta d \phi \\
& =p_{r} p_{\theta} R^{2} \int_{0}^{\pi} F_{1}(\theta) F_{2}(\theta) \sin \theta d \theta \int_{0}^{2 \pi} \cos \phi d \phi
\end{aligned}
$$

The integral of cos $\phi$ over $2 \pi$ is zero; therefore, the inner product is zero and the two functions are orthogonal.

$$
\begin{aligned}
& \text { Similarly, the inner product of } v_{r} \text { and } v_{\phi} \text { is } \\
& \left\langle v_{r} \mid v_{\phi}\right\rangle=p_{r} p_{\phi} R^{2} \int_{0}^{\pi} F_{1}(\theta) F_{2}(\theta) \sin \theta d \theta \int_{0}^{2 \pi} \sin \phi d \phi \quad \text { A30 }
\end{aligned}
$$

which is also zero since the integral of sinf over $2 \pi$ is zero. 
Finally, the inner product of $v_{\theta}$ and $v_{\phi}$ is

$$
v_{\theta} v_{\phi}=p_{\theta} p_{\phi} R^{2} \int_{0}^{\pi} F_{2}(\theta) \sin \theta d \theta \int_{0}^{2 \pi} \sin \phi \cos \phi d \phi \quad \text { A } 31
$$

Again, the integral of the product of $\sin \phi$ and $\cos \phi$ over $2 \pi 1 s$ zero, so these functions are also orthogonal. Therefore, the three functions $v_{r}, v_{\theta}$, and $v_{\phi}$ are mutualiy or thogonal. 
APPENDIX B. COMPUTER PROGRAMS AND OUTPUTS

In this appendix the programs used to simulato the matched filter are presented along with the output data. This data is also presented in the form of graphs in the main body of this thesis.

The flrat program was used to calculate the relative surface potentials for radiol and tangential dipoles. The method used is a stralghtforward eveluation of equations A15 through A2l of appendix A. The magnitude of the dipole component is assumed to be unity, and the constant $1 /(4 \pi \sigma R)$ is also set equal to unity. Since the variation with $f$ is sinusoidal, this variable is ignored and the voltages are calculated as a function of radial position, $d$, and $\theta$ only. This program was written in Hewlett-Packard Basic. Referring to the listing in figure B-l, variable L9 determines whether a radial or tangential dipole is assumed. If L9 is false (zero), potentials aro calculated for a radial dipole; if it is true (nonzero), a tangential dipole is used. Variables $\theta, d, S$, and $T$ of the equations are represented in the program by $Q, D, S I$, and $T l$, respectively.

Outputs for radial and tangential dipoles are 


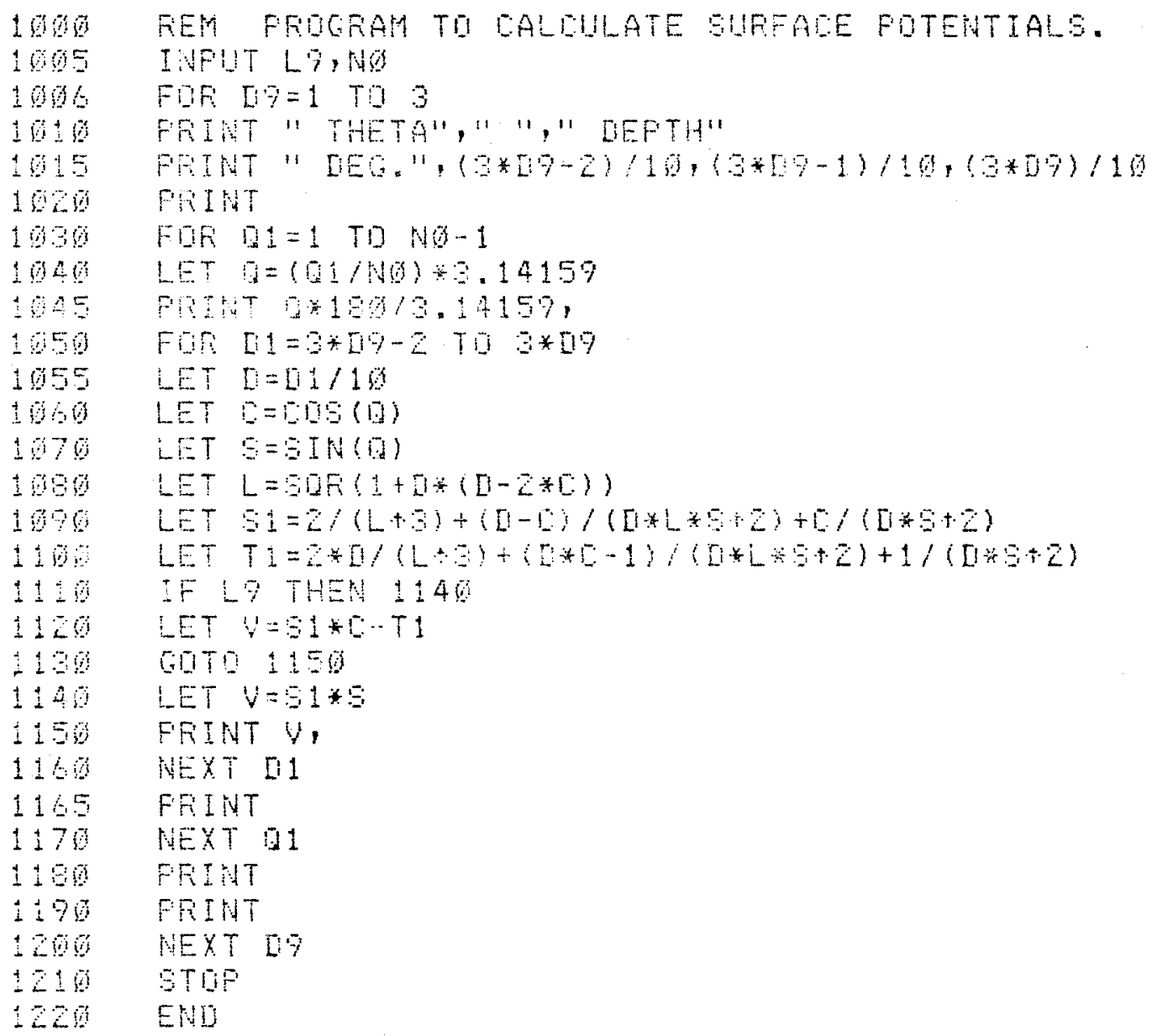

FIGURE BI. Program to calculate theoretical surfaco potentials. Potentials produced by radial and tangential dipoles are calculated using the single sphere model. 


\begin{tabular}{|c|c|}
\hline $\begin{array}{l}\text { THETA } \\
\text { DEG. }\end{array}$ & .1 \\
\hline 7 & 3.51956 \\
\hline 8 & 9.98773 \\
\hline 27 & 3.64978 \\
\hline 36 & 2.67332 \\
\hline 45 & 2.22096 \\
\hline 54 & 1.74261 \\
\hline 69 & 1.2336 \\
\hline 72 & $=722295$ \\
\hline 81 & .224730 \\
\hline 90 & -.24661 \\
\hline 99 & .4829 \\
\hline 268 & $\cdots 1,07797$ \\
\hline 117 & -1.42010 \\
\hline 126 & -1.79148 \\
\hline 135 & -1.7876 \\
\hline 144 & -2.19535 \\
\hline 150 & -2.3565 \\
\hline 162 & -2.47076 \\
\hline 171 & -2.5391 \\
\hline
\end{tabular}

DEFTH

.2

4.26705

3.75071

3.4926

2.91615

2.28555

1.64722

1.63597

.471657

$-9.275525-32$

- .474241

$-.659794$

$-1.17598$

$-1.4450$

$-1.66697$

$-1.84515$

$-1.98555$

$-2.69112$

$-2.1646$

$-2.20798$
.3

5.21994

4.77065

3.99510

2.11910

2.25675

1. 45769

.767696

.172948

. 202346

- .67921

. .976449

-1. 22789

$-1.42503$

$-1.5828$

$-1.76547$

$-1.79932$

$-1.06847$

$-1.91598$

$-1.74357$

\section{THETA}

DEG.

7

18

27

36

45

54

63

72

a 1

70

97

100

117

126

185

14

150

162

171
.4

6.0045

5.9279

4. 52222

a. 21673

2.6894

1. 16574

.452497

$-9.19461 E-02$

$-.505187$

$-.81905$

$-1.05550$

$-1.242$

$-1.2891$

$-1.4711$

$-1.57565$

.1 .6956

$-1.60609$

$-1.71110$

$-1.72869$

\section{DEFTH \\ .5}

7.16516

7.17715

4.97967

9.12223

1.7479

.793514

.112699

$-.256591$

- . .8053

$-.726696$

$-1=10060$

$-1.22942$

$-1.32564$

$-1.07795$

$-1.45214$

- 1.49231

$-1.52119$

$-1.54064$

$-1.55159$ .$t$

12.932

9.76635

$5=1956 \%$

2.72677

2. 2474

.247674

$-.217481$

$-.592448$

$-.826273$

.97422

.1 .11269

.1 .19828

- 1. 26092

$-1.0079$

$-1.34167$

$-1.96,61$

-1. .3649

$-1.29702$

$-1.48977$

FIGURE B2. Theoretical surface potentials of a radial dipole. (Page 1 of 2) 


\begin{tabular}{|c|c|c|c|}
\hline $\begin{array}{l}\text { THETA } \\
\text { DEG. }\end{array}$ & .7 & $\begin{array}{l}\text { DEFTH } \\
.9\end{array}$ & .9 \\
\hline 7 & 19.9186 & 27.6009 & 95.4922 \\
\hline 18 & 10.1151 & 7.80915 & 5.75987 \\
\hline 27 & 4.66906 & 3.28928 & 1.14373 \\
\hline 36 & 1.98167 & .765157 & -.107067 \\
\hline 4.5 & .6918 & $-7.45759 E-63$ & -.574746 \\
\hline 54 & $-9.14044 E-62$ & -.456055 & -.787371 \\
\hline 63 & -.566662 & -.79467 & -.897764 \\
\hline 72 & -.758480 & -.892987 & -.960732 \\
\hline 81 & -.720169 & -.775918 & -.997396 \\
\hline 90 & -1.62797 & -1.60574 & -1.0442 \\
\hline 79 & -1.10247 & -1.37655 & $-1.041: 2$ \\
\hline 108 & -1.15528 & -1.10569 & -1.05811 \\
\hline 117 & -1.19347 & -1.1260 & $-1,66159$ \\
\hline 26 & -1.22144 & -1.14144 & $-1 \cdot 36765$ \\
\hline 135 & -1.24202 & -1.15246 & -1.67211 \\
\hline 144 & -1.25769 & -1.16049 & -1.675 .5 \\
\hline 153 & -1.26771 & -1.16616 & -1.07764 \\
\hline 102 & -1.27455 & -1.16995 & -1.67917 \\
\hline 171 & -1.27873 & -1.17212 & -1.05004 \\
\hline
\end{tabular}

FIGURE B2. Theoretical surface potentials of a radial dipole. (Page 2 of 2) 


\section{THETA}

[EEG.

7

18

27

36

45

54

63

72

81

90

99

100

117

126

195

144

153

162

171

THETA

\section{DES.}

7
19
27
36
45
54
63
72
61
76
79
168
117
126
195
144
158
162
171

.1

.610298

1.19167

1.71899

2.17117

2.53458

2.90226

2. 97945

3.65283

3. 154646

2.76 .51

2.81954

2.61912

2.07977

2.97216

1.78187

1.44945

1.1005

.797856

.371729
DEFTH

.2

.022946

1.57887

2.21499

2.6999

3.02563

3. 26204

3.2479

3.19416

3.0592

2.86631

2.63272

2.37161

2.07266

1. 8027

1. 506.37

1. 2066.7

.905427

.603663

.901512

JEFTH

.5

2.78238

4.59726

5.27162

5.18075

4.74041

4.18675

3.644

0.14771

2.7694

2.32551

$\pm .7963$

1. 68987

1. 42253

1. $166 \%$

.957055

.75916

.552585

.96402

.197772
.2

1. 16057

2.16523

2.91303

3.97615

3.58478

9.57654

3.47173

3. 26069

3.00028

2.71531

2.42136

2.12757

1.98984

1. 55796

1. 28367

1. 01731

.757217

.502024

.250166

6

4.92484

7.12252

7.09021

6.18593

5.17986

4.29669

3.57276

2.96766

2.51162

2.11851

1.78796

1.56464

1. 25716

1. 63091

.85734

.653319

.490758

.316254

.42446

21105

FIGURE B3. Theoretical surfoco potentials of a tangential dipola. (Page 1 of 2) 


\begin{tabular}{|c|c|c|c|}
\hline $\begin{array}{l}\text { THETA } \\
\text { DEG. }\end{array}$ & .7 & $\begin{array}{l}\text { DEFTH } \\
.5\end{array}$ & .9 \\
\hline 9 & 9.70637 & 23.2036 & $57.788 \%$ \\
\hline 18 & 11.2797 & 17.259 & 22.9485 \\
\hline 27 & 7.18250 & 11.0509 & 11.7271 \\
\hline 36 & 7.02555 & 7.47468 & 7.40535 \\
\hline 45 & 5.40767 & 5.3789 & 5.11637 \\
\hline 54 & 4.25014 & 4.06566 & 3.78361 \\
\hline 63 & 9.41097 & 0.1954 & 2.72572 \\
\hline 72 & 2.79472 & 2.56099 & 2.39255 \\
\hline$\theta 1$ & 2.36223 & 2.69467 & 1.898 \\
\hline 96 & 1.91097 & 1.79315 & 1.56462 \\
\hline 97 & 1.66546 & 1.4425 & 1,27881 \\
\hline 100 & 1.3424 & 1.20153 & 1.67979 \\
\hline 117 & 1.11621 & .996246 & 974650 \\
\hline 126 & .917313 & .816974 & .732425 \\
\hline 135 & .736737 & .656906 & .589451 \\
\hline 144 & .575222 & .516705 & .457402 \\
\hline 153 & .42267 & .375695 & .955676 \\
\hline 162 & .277766 & .246362 & 220412 \\
\hline 171 & .137769 & .122096 & .109221 \\
\hline
\end{tabular}

FIGURE B3. Theoretical surface potentials of a tangential dipole. (Page 2 of 2$)$ 
shown in figures $B-2$ and $B-3$. Plots of these deta are shown in chapter III, flgures III-1 and III-2.

\section{DEPTH MI SMATCH SIMULATIONS}

Two programs were written to calculate the theoretical effect of depth mismetch on the filter output (see figures $B-4$ and $B-7$ for program listings). These programs eveluate the functions $b_{r}, b_{\theta}$, or $b_{\phi}$ as defined in eq. II-36. The function $\nabla(\theta, \phi)$ in these equations is the surface potential of a current dipole on the $z$ axis having the same orientation as the dipole to which the filter is matchod.

The equations for the single sphere model from appendix A are again used with the component dipole magnitude, the radius $R$, and the constant $1 /(4 \pi \sigma R)$ all set equal to one. For a radial dipole, the integral to be evaluated is

$$
b_{r}=\int b_{r}(\theta, \phi) v(\theta, \phi) d q
$$

Using equation A26, this equation becomes

$$
b_{r}=\frac{1}{c_{r}} \int_{0}^{2 \pi} \int_{0}^{\pi} F_{1}\left(\theta \mid r_{1}\right) F_{1}\left(\theta \mid r_{0}\right) R^{2} \sin \theta d \theta d \phi
$$

whore $r_{0}$ and $r_{1}$ are the radial position coordinates of the actual dipole and the filter dipole, respectively. (dependence of $F_{1}$ on the other dipolo parameters is not 


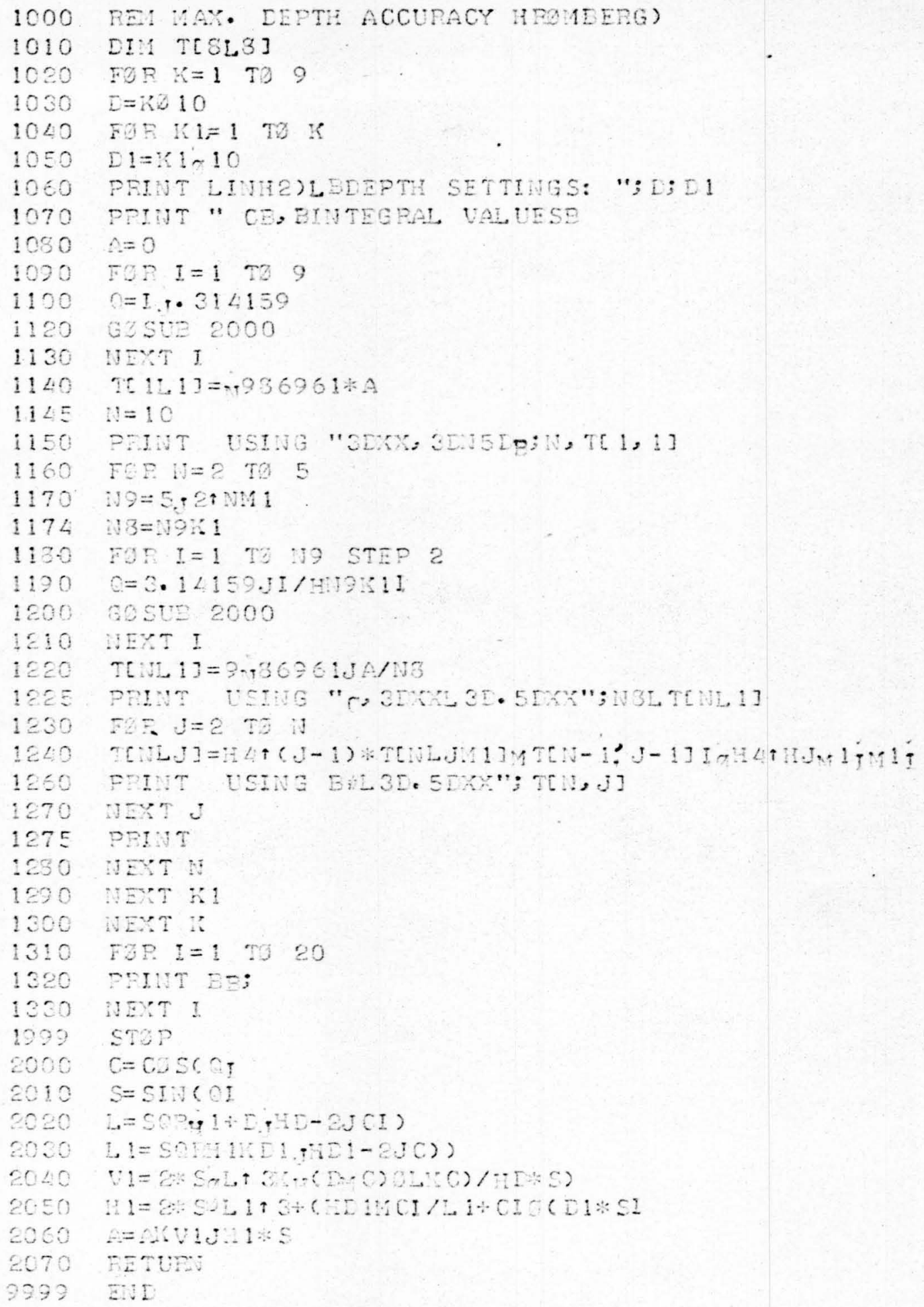

FIGURE B4. Program to test numerical integration for depth mismatch. 


\begin{tabular}{|c|c|c|c|c|c|}
\hline $\begin{array}{l}\text { DEPTH } \\
10 \\
10 \\
20 \\
40 \\
80 \\
160\end{array}$ & $\begin{array}{l}\text { SETTINGS: } \\
37.32111 \\
38.08510 \\
38.27379 \\
38.32059 \\
38.33242\end{array}$ & $\begin{array}{l}38 \cdot 33976 \\
38 \cdot 33656 \\
38.33636 \\
38 \cdot 33633\end{array}$ & $\begin{array}{l}38.33635 \\
38.33634 \\
38.33633\end{array}$ & $\begin{array}{l}38 \cdot 33634 \\
38 \cdot 33633\end{array}$ & $38 \cdot 33633$ \\
\hline $\begin{array}{c}\text { DEPTH } \\
4 \\
10 \\
20 \\
40 \\
80 \\
160\end{array}$ & $\begin{array}{l}\text { SETTINGS: } \\
37.87926 \\
38.71487 \\
38.92282 \\
38.97462 \\
38.98756\end{array}$ & $\begin{array}{l}\text {-2 } \\
\text { ITEGRAL VA } \\
38.99641 \\
38.99214 \\
38.99189 \\
38.99187\end{array}$ & $\begin{array}{l}38.99185 \\
38.99187 \\
38.99186\end{array}$ & $\begin{array}{l}38.99187 \\
38.99186\end{array}$ & 38.99186 \\
\hline $\begin{array}{c}\text { DEPTH } \\
10 \\
10 \\
20 \\
40 \\
80 \\
160\end{array}$ & $\begin{array}{l}\text { SETTINGS: } \\
39.09229 \\
40.04820 \\
40.28287 \\
40.34128 \\
40.35589\end{array}$ & $\begin{array}{l}.2 \\
\text { JTEGRAL VA } \\
40.36684 \\
40.36169 \\
40.36075 \\
40.36076\end{array}$ & $\begin{array}{l}40.36071 \\
40.36073 \\
40.36076\end{array}$ & $\begin{array}{l}40.36073 \\
40.35076\end{array}$ & 40.36076 \\
\hline $\begin{array}{c}\text { DEPTH } \\
10 \\
10 \\
20 \\
40 \\
80 \\
160\end{array}$ & $\begin{array}{l}\text { SETTINGS: } \\
38.36150 \\
39.34529 \\
39.58646 \\
39.64646 \\
39.66142\end{array}$ & $\begin{array}{l}.3 \\
\text { JTEGRAL VA } \\
39.67323 \\
39.66685 \\
39.66647 \\
39.66641\end{array}$ & $\begin{array}{l}39.66642 \\
39.66644 \\
39.66640\end{array}$ & $\begin{array}{l}39.66644 \\
39.66540\end{array}$ & 39.65640 \\
\hline $\begin{array}{c}\text { DEPTH } \\
10 \\
20 \\
40 \\
80 \\
160\end{array}$ & $\begin{array}{l}\text { SETTINGS: } \\
40.30577 \\
41.44241 \\
41.72005 \\
41.78908 \\
41.80630\end{array}$ & $\begin{array}{l}\text {.3 } \\
\text { ITEGRAL VA } \\
41.82128 \\
41.81261 \\
41.81209 \\
41.81205\end{array}$ & $\begin{array}{l}41.81203 \\
41.81205 \\
41.81204\end{array}$ & $\begin{array}{l}41.81205 \\
41.81204\end{array}$ & 41.81204 \\
\hline $\begin{array}{c}\text { DEPTH } \\
10 \\
10 \\
20 \\
40 \\
80 \\
160\end{array}$ & $\begin{array}{l}\text { SETTINGS: } \\
42.33932 \\
43.71443 \\
44.04852 \\
44.13147 \\
44.15218\end{array}$ & $\begin{array}{l}\text {.3 } \\
\text { JTEGRAL VA } \\
44.17280 \\
44.15989 \\
44.15912 \\
44.15909\end{array}$ & $\begin{array}{l}44.15903 \\
44.15907 \\
44.15909\end{array}$ & $\begin{array}{l}44 \cdot 15907 \\
44 \cdot 15909\end{array}$ & $44 \cdot 15909$ \\
\hline
\end{tabular}




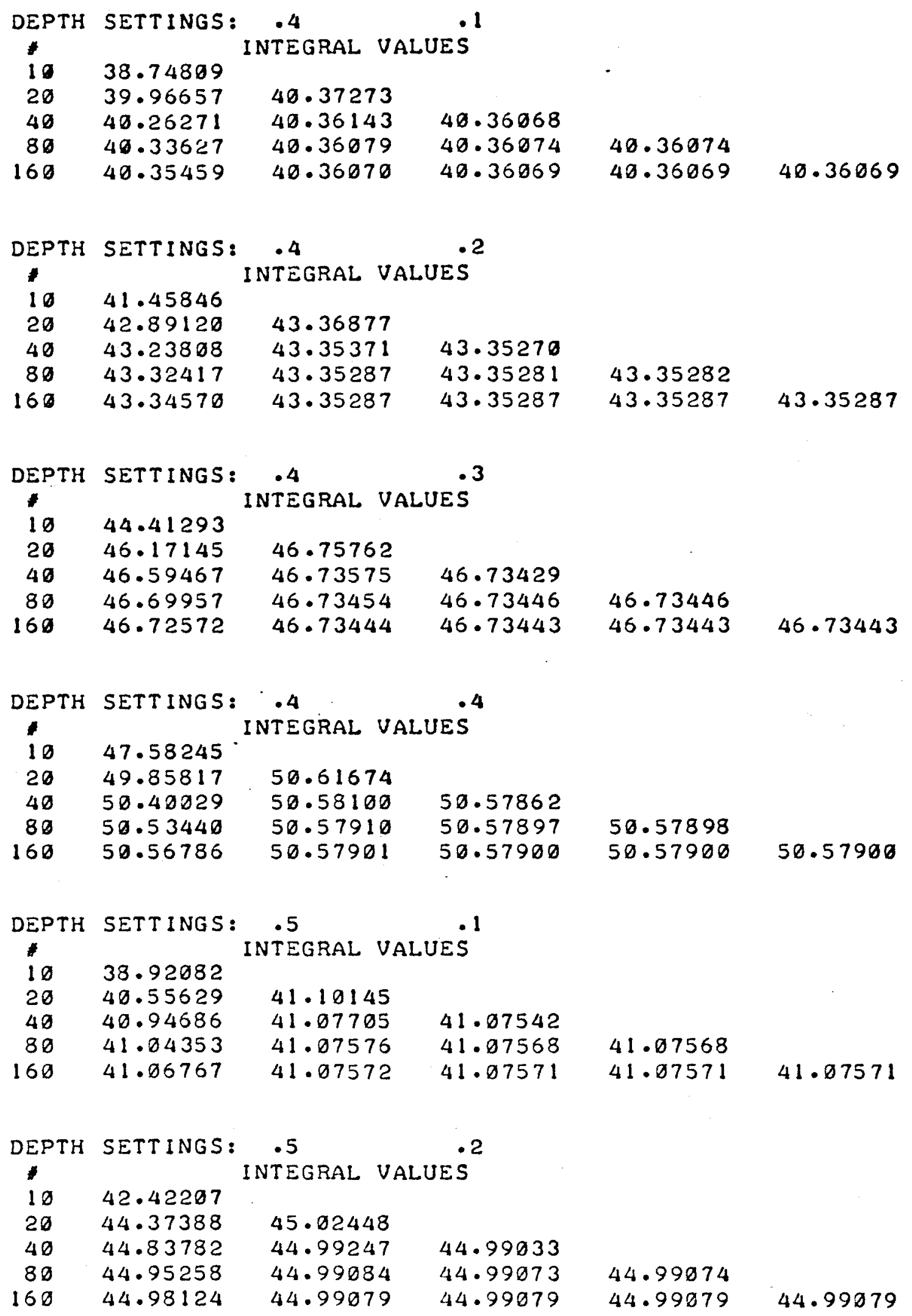

FIGURE B5. Integral values for depth mismatch with a radial dipole. (Page 2 of 8 ) 


\begin{tabular}{|c|c|c|c|c|c|}
\hline DEPTH & SETT INGS: & .5 & .3 & & \\
\hline+ & & NTEGRAL VA & UES & & \\
\hline 10 & 46.38442 & & & & \\
\hline 20 & 48.80902 & 49.61722 & & & \\
\hline 40 & 49.38142 & 49.57223 & 49.56923 & & \\
\hline 80 & 49.52281 & 49.56995 & 49.56979 & 49.56980 & \\
\hline 160 & 49.55807 & 49.56982 & 49.56982 & 49.56982 & 49.56982 \\
\hline DEPTH & SETTINGS: & .5 & .4 & & \\
\hline$\#$ & & NTEGRAL VA & UES & & \\
\hline 10 & 50.82604 & & & & \\
\hline 20 & 53.99547 & 55.05194 & & & \\
\hline 40 & 54.73505 & 54.98158 & 54.97689 & & \\
\hline 80 & 54.91736 & 54.97813 & 54.97790 & 54.97791 & \\
\hline 160 & 54.96277 & 54.97791 & 54.97789 & 54.97789 & 54.97789 \\
\hline DEPTH & SETTINGS: & .5 & .5 & & \\
\hline & & NTEGRAL VA & JES & & \\
\hline & $\begin{array}{l}55.63528 \\
60.08755\end{array}$ & 61.57163 & & & \\
\hline 40 & 61.10333 & 61.44193 & 61.43327 & & \\
\hline 80 & 61.35284 & 61.43601 & $61 \cdot 43562$ & 61.43566 & \\
\hline 160 & 61.41493 & 61.43563 & $61 \cdot 43560$ & 61.43560 & 61.43560 \\
\hline DEPTH & SETTINGS: & .6 & .1 & & \\
\hline & & NTEGRAL VA & JES & & \\
\hline 10 & 38.58018 & & & & \\
\hline 20 & 41.85865 & 41.88480 & & & \\
\hline 40 & 41.62622 & 41.81541 & 41.81079 & & \\
\hline 80 & 41.76578 & 41.81229 & 41.81209 & 41.81211 & \\
\hline 160 & 41.80050 & 41.81207 & 41.81206 & 41.81206 & 41.81206 \\
\hline & & & & & \\
\hline DEPTH & SETTINGS: & .6 & $\cdot 2$ & & \\
\hline & & NTEGRAL VA & JES & & \\
\hline 10 & 42.83566 & & & & \\
\hline 20 & 45.82964 & 46.82763 & & & \\
\hline 40 & 46.51146 & 46.73874 & 46.73281 & & \\
\hline 80 & 46.67893 & 46.73476 & 46.73450 & 46.73452 & \\
\hline 160 & 46.72057 & 46.73446 & 46.73444 & 46.73444 & 46.73444 \\
\hline DEPTH & SETT INGS: & .6 & .3 & & \\
\hline 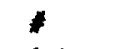 & & ITEGRAL VA & JES & & \\
\hline 10 & 47.82143 & & & & \\
\hline 20 & 51.57694 & 52.82879 & & & \\
\hline 40 & 52.42540 & 52.70822 & 52.70019 & & \\
\hline 80 & 52.63352 & 52.70290 & 52.70255 & 52.70258 & \\
\hline 160 & 52.68532 & 52.70258 & 52.70256 & 52.70256 & 52.70256 \\
\hline
\end{tabular}

FIGURE B5. Integral values for depth mismatch with a radial dipole. (Page 3 of 8 ) 


\begin{tabular}{|c|c|c|c|c|c|}
\hline $\begin{array}{l}\text { DEPTH } \\
10 \\
10 \\
20 \\
40 \\
80 \\
160\end{array}$ & $\begin{array}{l}\text { SETTINGS: } \\
53.63584 \\
58.58380 \\
59.68745 \\
59.95760 \\
60.02473\end{array}$ & $\begin{array}{l}60.23312 \\
60.05534 \\
60.04765 \\
60.04712\end{array}$ & $\begin{array}{l}60.04348 \\
60.04713 \\
60.04709\end{array}$ & $\begin{array}{l}60.04720 \\
60.04708\end{array}$ & 60.04708 \\
\hline $\begin{array}{c}\text { DEPTH } \\
10 \\
10 \\
20 \\
40 \\
80 \\
160\end{array}$ & $\begin{array}{l}\text { SETT INGS: } \\
60.23586 \\
67.22510 \\
68.74875 \\
69.12033 \\
69.21262\end{array}$ & $\begin{array}{l}.6 \\
\text { NTEGRAL VA } \\
69.55484 \\
69.25664 \\
69.24419 \\
69.24338\end{array}$ & $\begin{array}{l}69.23676 \\
69.24336 \\
69.24333\end{array}$ & $\begin{array}{l}69.24347 \\
69.24333\end{array}$ & 69.24333 \\
\hline
\end{tabular}

$\begin{array}{llllll}\text { DEPTH } & \text { SETTINGS: } & .6 & .6 & \\ 10 & 67.00139 & & & \\ 10 & & & & \\ 20 & 77.96053 & 81.61357 & & & \\ 40 & 80.25545 & 81.02043 & 80.98090 & & \\ 80 & 80.81076 & 80.99586 & 80.99422 & 80.99443 & \\ 160 & 80.94852 & 80.99443 & 80.99434 & 80.99434 & 80.99434\end{array}$

\begin{tabular}{llllll} 
DEPTH & SETTINGS: & .7 & & \\
* & \multicolumn{1}{l}{ INTEGRAL VALUES } & & \\
10 & 36.79640 & & & & \\
20 & 41.30150 & 42.80320 & & & \\
40 & 42.26224 & 42.58248 & 42.56776 & & \\
80 & 42.49416 & 42.57146 & 42.57072 & 42.57077 & \\
160 & 42.55169 & 42.57087 & 42.57083 & 42.57083 & 42.57083
\end{tabular}

\begin{tabular}{cccccc} 
DEPTH & SETTINGS: & .7 & & \\
& \multicolumn{2}{l}{${ }^{2}$} & & \\
10 & 41.57220 & INTEGRAL VALUES & & \\
20 & 47.05629 & 48.88432 & & & \\
40 & 48.22025 & 48.50824 & 48.58984 & & \\
80 & 48.50091 & 48.59446 & 48.59354 & 48.59360 & \\
160 & 48.57049 & 48.59368 & 48.59363 & 48.59363 & 48.59363
\end{tabular}

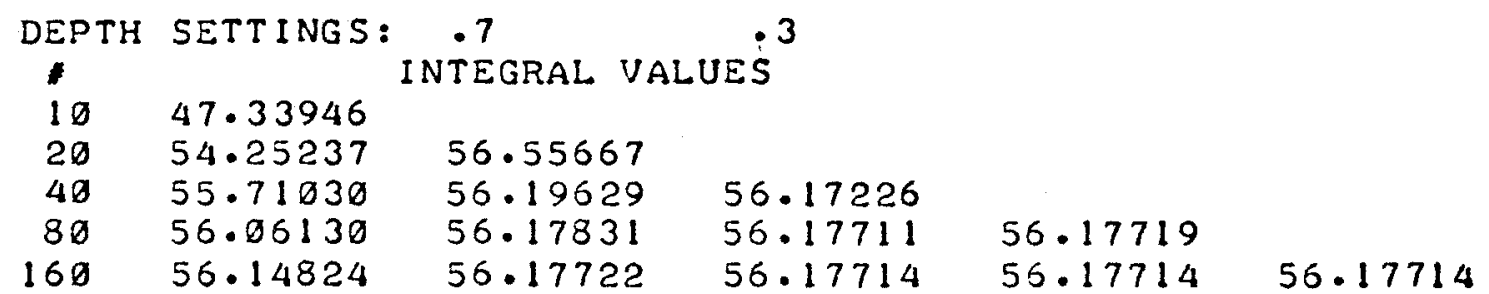

FIGURE B5. Integral values for depth mismatch with a radial dipole. (Page 4 of 8 ) 


\begin{tabular}{|c|c|c|c|c|c|}
\hline 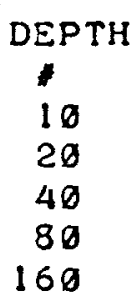 & $\begin{array}{l}\text { SETTINGS: } \\
54.29671 \\
63.41976 \\
65.32584 \\
65.78358 \\
65.89694\end{array}$ & $\begin{array}{l}66.46078 \\
65.96120 \\
65.93617 \\
65.93474\end{array}$ & $\begin{array}{l}65.92789 \\
65.93451 \\
65.93465\end{array}$ & $\begin{array}{l}65.93462 \\
65.93465\end{array}$ & 65.93465 \\
\hline $\begin{array}{c}\text { DEPTH } \\
10 \\
20 \\
40 \\
80 \\
160\end{array}$ & $\begin{array}{l}\text { SETTINGS: } \\
62.50282 \\
75.33609 \\
77.97847 \\
78.61035 \\
78.76669\end{array}$ & $\begin{array}{l}.7 \\
\text { INTEGRAL VA } \\
79.61385 \\
78.85927 \\
78.82098 \\
78.81880\end{array}$ & $\begin{array}{l}78.80898 \\
78.81842 \\
78.81865\end{array}$ & $\begin{array}{l}78.81857 \\
78.81865\end{array}$ & 78.81865 \\
\hline $\begin{array}{l}\text { DEPTH } \\
10 \\
10 \\
20 \\
40 \\
80 \\
160\end{array}$ & $\begin{array}{l}\text { SETTINGS: } \\
71.34486 \\
91 \cdot 12369 \\
95 \cdot 11847 \\
96.06551 \\
96.29955\end{array}$ & $\begin{array}{l}.7 \\
\text { INTEGRAL VA } \\
97.71663 \\
96.45007 \\
96.38118 \\
96.37756\end{array}$ & $\begin{array}{l}96 \cdot 36565 \\
96 \cdot 37660 \\
96 \cdot 37733\end{array}$ & $\begin{array}{l}96 \cdot 37679 \\
96 \cdot 37733\end{array}$ & 96.37735 \\
\hline $\begin{array}{l}\text { DEPTH } \\
4 \\
10 \\
20 \\
40 \\
80 \\
160\end{array}$ & $\begin{array}{l}\text { SETTINGS: } \\
77.85780 \\
112.13425 \\
119.11887 \\
120.73689 \\
121.13521\end{array}$ & $\begin{array}{l}.7 \\
\text { INTEGRAL VA } \\
123.55974 \\
121.44798 \\
121.27625 \\
121.26799\end{array}$ & $\begin{array}{l}121.30623 \\
121.26486 \\
121.26744\end{array}$ & $\begin{array}{l}121 \cdot 26421 \\
121 \cdot 26749\end{array}$ & 121.26749 \\
\hline $\begin{array}{c}\text { DEPTH } \\
10 \\
20 \\
40 \\
80 \\
160\end{array}$ & $\begin{array}{l}\text { SETTINGS: } \\
31.04420 \\
40.48463 \\
42.69365 \\
43.19066 \\
43.31245\end{array}$ & $\begin{array}{l}.8 \\
\begin{array}{l}8 \\
43.63145 \\
43.42999 \\
43.35633 \\
43.35386\end{array}\end{array}$ & $\begin{array}{l}43.41656 \\
43.35143 \\
43.35284\end{array}$ & $\begin{array}{l}43.35040 \\
43.35286\end{array}$ & 43.35287 \\
\hline $\begin{array}{c}\text { DEPTH } \\
10 \\
20 \\
40 \\
80 \\
160\end{array}$ & $\begin{array}{l}\text { SETTINGS: } \\
35.58067 \\
47.08428 \\
49.77675 \\
50.38166 \\
50.52980\end{array}$ & $\begin{array}{l}.8 \\
\text { INTEGRAL VA } \\
50.91882 \\
50.67423 \\
50.58331 \\
50.57919\end{array}$ & $\begin{array}{l}50.65793 \\
50.57725 \\
50.57891\end{array}$ & $\begin{array}{l}50.57597 \\
50.57894\end{array}$ & 50.57895 \\
\hline
\end{tabular}

FIGURE B5. Integral values for depth mismatch with a radial dipole. (Page 5 of 8 ) 


\begin{tabular}{|c|c|c|c|c|c|}
\hline $\begin{array}{l}\text { DEPTH } \\
10 \\
10 \\
20 \\
40 \\
80 \\
160\end{array}$ & $\begin{array}{l}\text { SETTINGS: } \\
41.18556 \\
55.65137 \\
59.03963 \\
59.79940 \\
59.98545\end{array}$ & $\begin{array}{l}60.47330 \\
60.16904 \\
60.05267 \\
60.04746\end{array}$ & $\begin{array}{l}60.14877 \\
60.04491 \\
60.04712\end{array}$ & $\begin{array}{l}50.04326 \\
60.04716\end{array}$ & 60.04717 \\
\hline $\begin{array}{l}\text { DEPTH } \\
10 \\
10 \\
20 \\
40 \\
80 \\
160\end{array}$ & $\begin{array}{l}\text { SETTINGS: } \\
48.11955 \\
67.06851 \\
71.51479 \\
72.50894 \\
72.75223\end{array}$ & $\begin{array}{l}\text {.8 } \\
\text { INTEGRAL VA } \\
73.38484 \\
72.99687 \\
72.84033 \\
72.83333\end{array}$ & $\begin{array}{l}72.97101 \\
72.82990 \\
72.83286\end{array}$ & $\begin{array}{l}72.82767 \\
72.83290\end{array}$ & 72.83292 \\
\hline $\begin{array}{l}\text { DEPTH } \\
: \\
10 \\
20 \\
40 \\
80 \\
160\end{array}$ & $\begin{array}{l}\text { SETTINGS: } \\
56.52834 \\
82.75034 \\
88.93369 \\
90.30942 \\
90.64572\end{array}$ & $\begin{array}{l}91.49101 \\
90.99481 \\
90.76801 \\
90.75783\end{array}$ & $\begin{array}{l}90.96173 \\
90.75288 \\
90.75714\end{array}$ & $\begin{array}{l}90.74957 \\
90.75720\end{array}$ & 90.75723 \\
\hline $\begin{array}{l}\text { DEPTH } \\
1 \\
10 \\
20 \\
40 \\
80 \\
160\end{array}$ & $\begin{array}{l}\text { SETTINGS: } \\
65.90335 \\
105.00052 \\
114.37607 \\
116.44173 \\
116.94577\end{array}$ & $\begin{array}{l}\stackrel{8}{\text { INTEGRAL VA }} \\
\begin{array}{l}118.03291 \\
117.50127 \\
117.13029 \\
117.11378\end{array}\end{array}$ & $\begin{array}{l}117.46582 \\
117.10556 \\
117.11269\end{array}$ & $\begin{array}{l}117.09984 \\
117.11281\end{array}$ & 117.11285 \\
\hline $\begin{array}{l}\text { DEPTH } \\
1 \\
10 \\
20 \\
40 \\
80 \\
160\end{array}$ & $\begin{array}{l}\text { SETTINGS: } \\
73.39093 \\
137.28430 \\
153.69971 \\
157.23471 \\
158.09372\end{array}$ & $\begin{array}{l}.8 \\
\text { INTEGRAL VA } \\
158.58209 \\
159.17151 \\
158.41306 \\
158.38007\end{array}$ & $\begin{array}{l}159.21982 \\
158.36249 \\
158.37787\end{array}$ & $\begin{array}{l}158.34903 \\
158.37811\end{array}$ & 158.37820 \\
\hline $\begin{array}{l}\text { DEPTH } \\
10 \\
10 \\
20 \\
40 \\
80 \\
160\end{array}$ & $\begin{array}{l}\text { SETTINGS: } \\
78.48421 \\
180.75055 \\
218.62714 \\
226.36603 \\
228.22040\end{array}$ & $\begin{array}{l}.8 \\
\text { INTEGRAL VA } \\
217.50598 \\
231.25269 \\
228.94568 \\
228.83353\end{array}$ & $\begin{array}{l}232.16913 \\
228.79190 \\
228.83139\end{array}$ & $\begin{array}{l}228.73828 \\
228.83283\end{array}$ & \\
\hline
\end{tabular}

FIGURE B5. Integral values for depth mismatch with a radial dipole. (Page 6 of 8 ) 


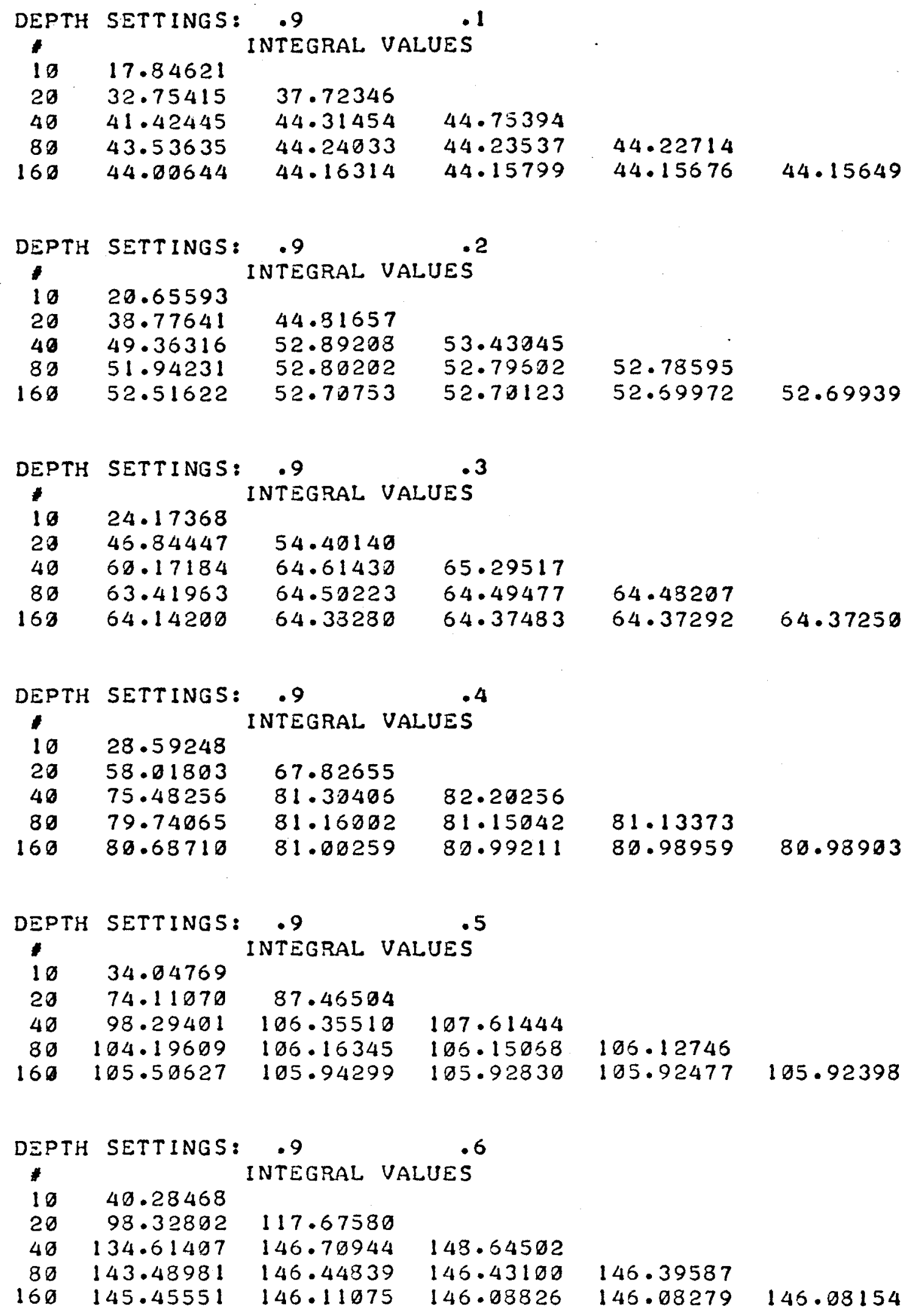

FIGURE B5. Integral values for depth mismotch with a radial dipolo. (Page 7 of 8 ) 


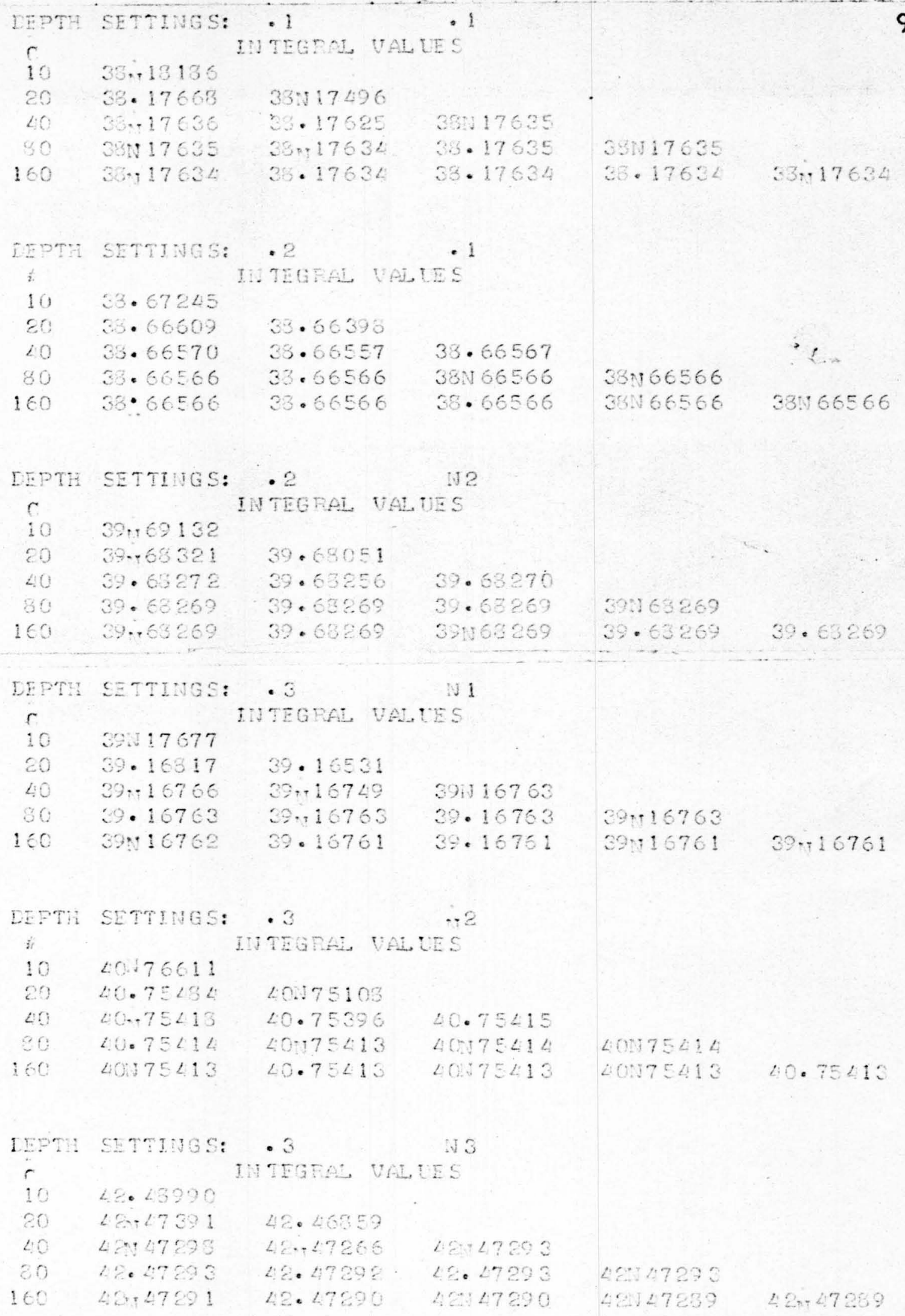

FIGURE B6. Integral values for depth mismatch with a tangentlal dipole. (Page 1 of 8 ) 


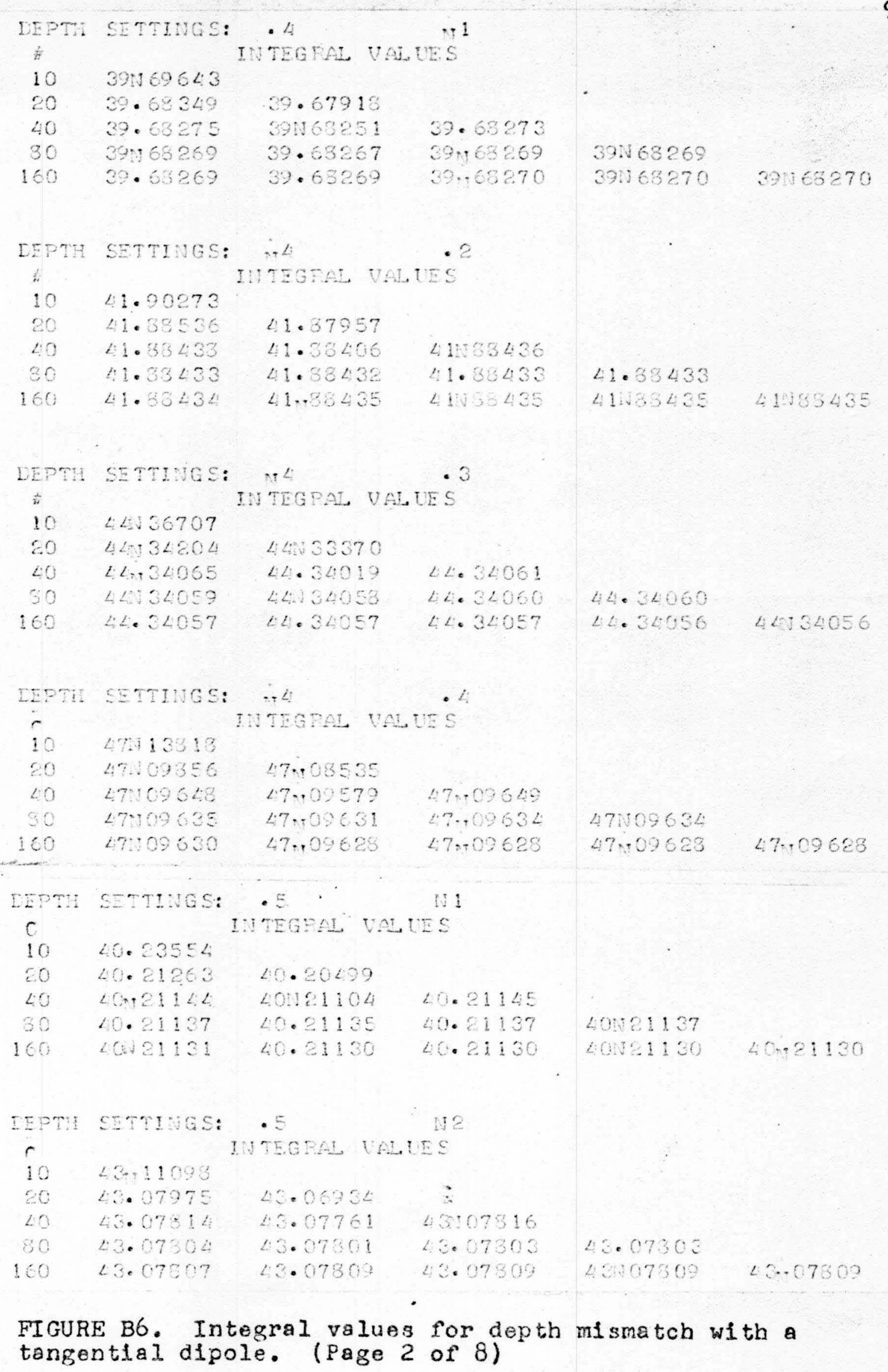

$\frac{1}{4}$

$1039 n 69643$

$20 \quad 39.68349$

$40 \quad 39.63275$

$30 \quad 39065269$

$160 \quad 3968269$

TNTEGPAL VAJ.UES

39.67918

$3946325 !$

39.68267

39.68273

39.683270

$39 N 68269$

$39: 163270$

39168270

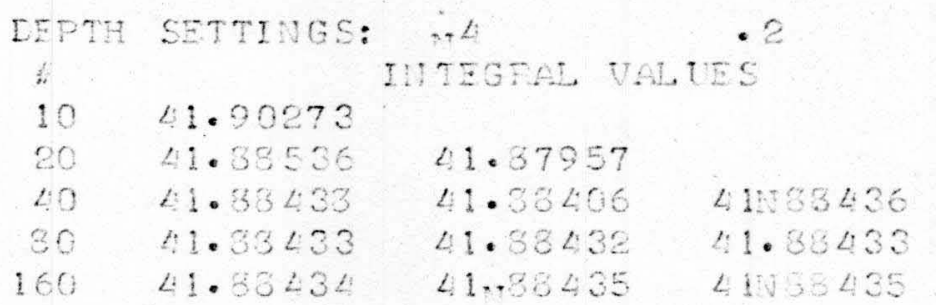

42.38433

$41183435 \quad 410353435$

DEPTH SETTINGS: NL

1044536707

$20 \quad 4434204$

$40 \quad \operatorname{Lin} 34065$

$50 \quad 42034059$

$160 \quad 42.34057$

44033370

44.34019

4434058

44.34057

$\angle \angle \cdot 34061$

42.34060

44.34057

$44 \cdot 34060$

$44.34056 \quad 4034056$

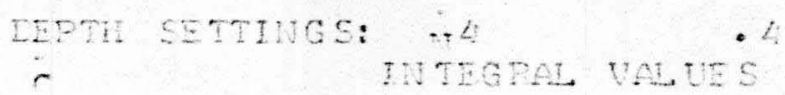

1047913318

$20 \quad 47.09356$

$40 \quad 47009648$

47.008535

9047009635

47000579

47,09631

$47+09649$

47,09628

4.009634

47,009628

47009634

47,009628

47,09688

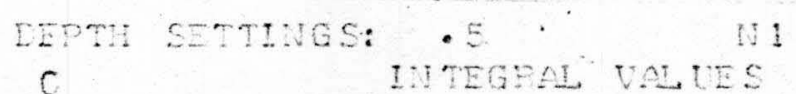

$10 \quad 40.23554$

co $40.21263 \quad 40.20299$

$\angle O \quad \angle O M E 1 L L \quad \angle 0 N E 1104$

$3040.21137 \quad 40.21135 \quad 40.21137$

$160 \quad 40421131$

$40.21130 \quad 40.21130$

40121137

$40151130 \quad 40 m 21130$

FIGURE B6. Integral values for depth mismatch with a tangentlal dipole. (Page 2 of 8 ) 


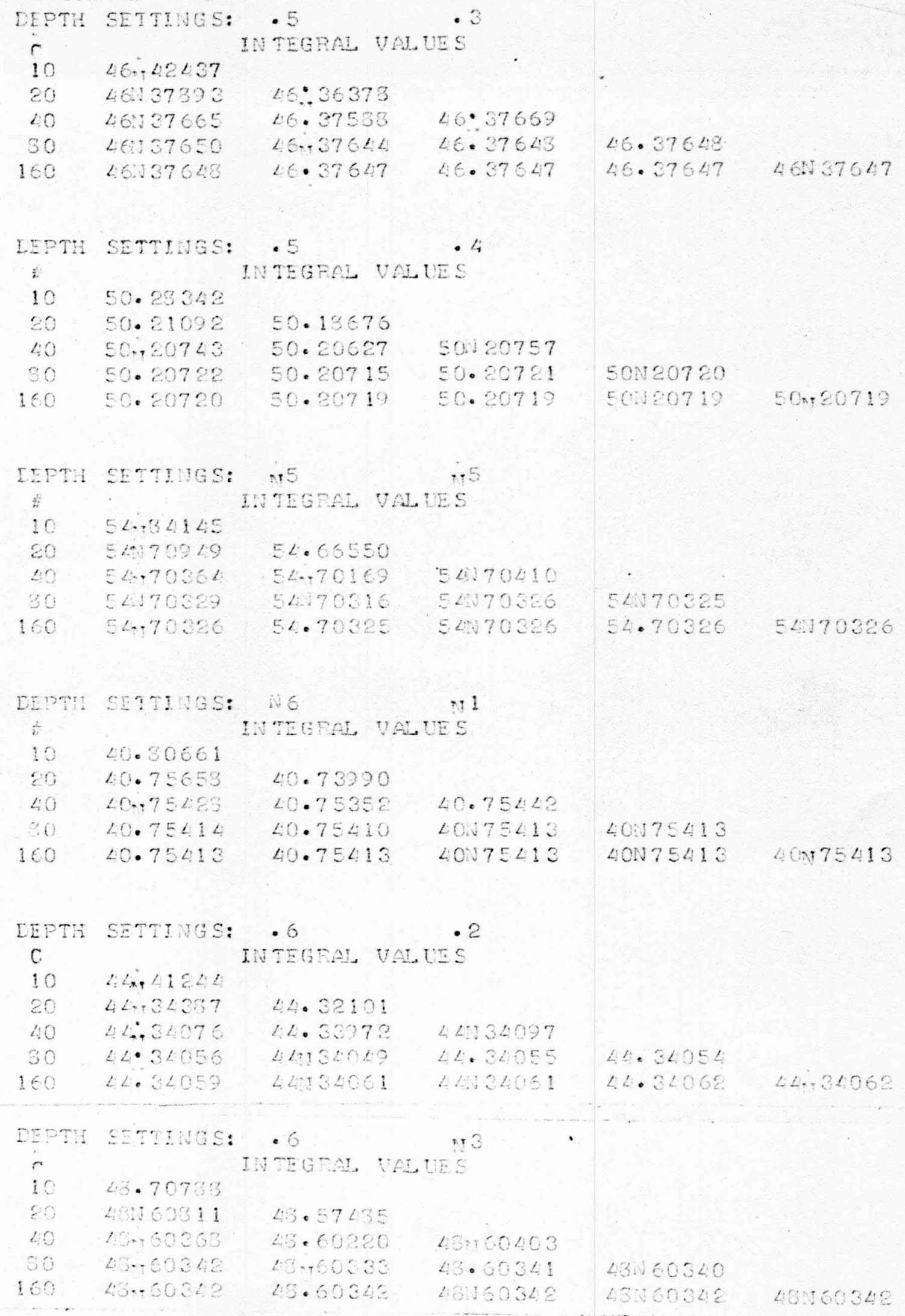

FIGURE B6. Integral values for depth mismatch with a tangential dipole. (Page 3 of 8 ) 


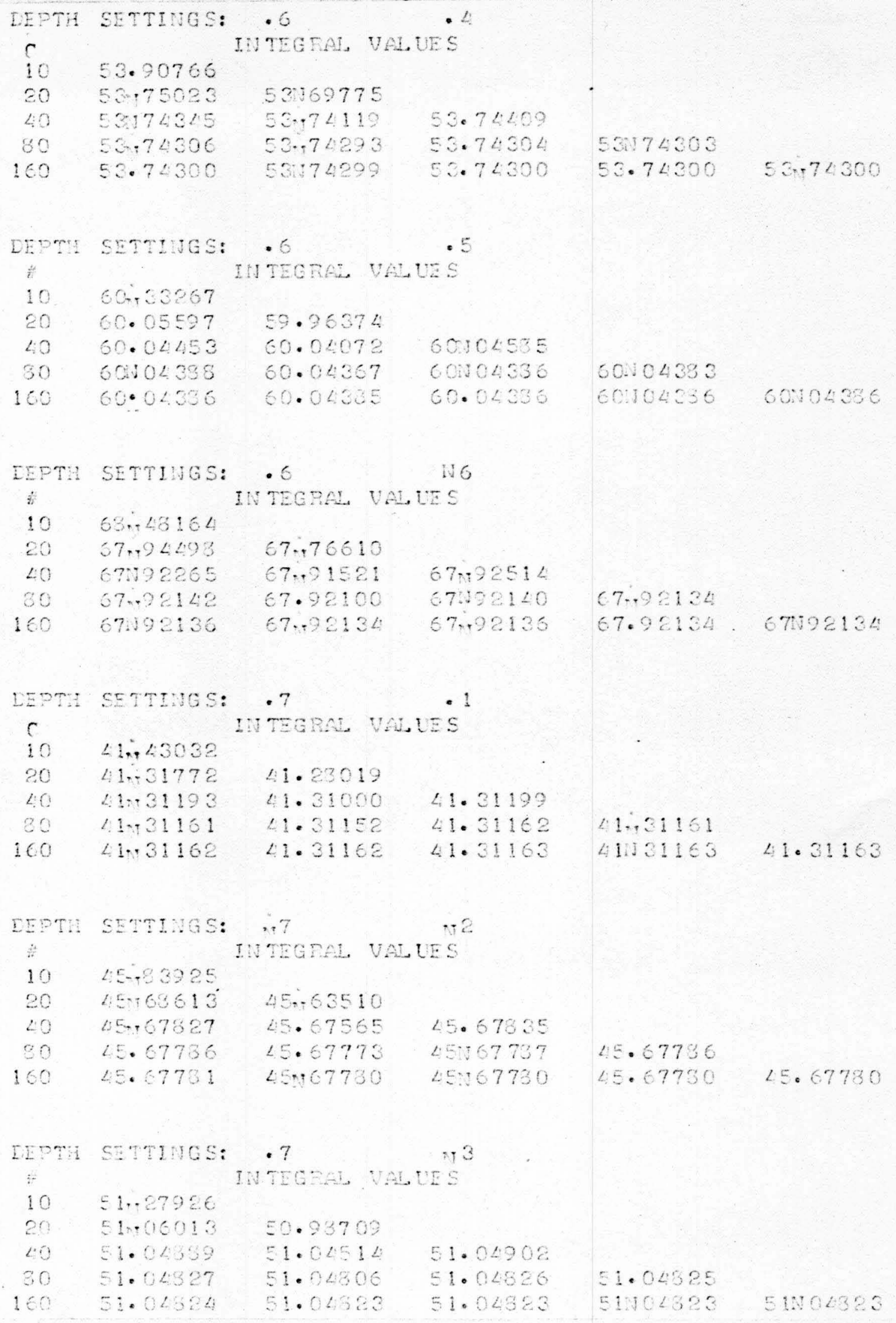

FIGURE B6. Integral values for depth mismatch with a tangential dipole. (Page 4 of 8 ) 


\begin{tabular}{|c|c|c|c|c|c|}
\hline DEPTH & SETTINGS: & 17 & .4 & & 95 \\
\hline $\begin{array}{l}\text { th } \\
10\end{array}$ & $58 N 14497$ & NTEGBAL V & UES & & 95 \\
\hline 20 & 57.81055 & 57,69907 & & & \\
\hline$\angle 0$ & 571179329 & $57 \times 73754$ & $57 \times 79343$ & & \\
\hline 80 & $57 \times 379236$ & 57,799205 & $57 \cdot 79236$ & 57.79234 & \\
\hline 160 & $57 N 79228$ & $57 \times 79226$ & 57.779227 & $57 N 79227$ & $57 N 79227$ \\
\hline EEPTH & SETTINGS: & .7 & N5 & & \\
\hline$\frac{t}{t}$ & 67.05649 & TEGEAL VA & IES & & \\
\hline 20 & 66150650 & 66432317 & & & \\
\hline 20 & $66 \cdot 47731$ & 66.266757 & $66 \times 47720$ & & \\
\hline 80 & 66.427580 & $66 \cdots 47530$ & $6674758:$ & 66747578 & \\
\hline 160 & $66: 47569$ & $66 \cdot 27566$ & $66 \times 47568$ & $66 \cdot 47563$ & 66.47568 \\
\hline IEPTE & SETPINGS: & $\frac{7}{\text { TEGRAL VA }}$ & : 6 & & \\
\hline 10 & 79000438 & & & & \\
\hline 20 & $73.06<33$ & 77075081 & & & \\
\hline$\angle 0$ & 78.00691 & 77.98773 & $78 \times 00359$ & & \\
\hline 30 & $78 \cdot 00404$ & 731000310 & $78 N 00 \angle 12$ & 76,00412 & \\
\hline 160 & $78 n 00389$ & $73 N 00304$ & 78000289 & 73000389 & $73 N 00339$ \\
\hline DEPTH & SETTHUG: & N7 & .7 & & \\
\hline ro & & II TEGRAL VA & $U E S$ & & \\
\hline $\begin{array}{l}10 \\
20\end{array}$ & $95-31302$ & & & & \\
\hline $\begin{array}{l}20 \\
40\end{array}$ & 94.06223 & 93064531 & & & \\
\hline $\begin{array}{l}40 \\
30\end{array}$ & $93 \cdot 91492$ & 9306533 & $93+88054$ & & \\
\hline $\begin{array}{r}30 \\
160\end{array}$ & 93.90331 & 93090611 & 93.00850 & 93.90924 & \\
\hline 160 & 93.90790 & 93.90776 & 93.90733 & 23,90787 & 93,9078 \\
\hline IEPTI & SPTINGS: & .8 & Ni & & \\
\hline 10 & 41.99715 & TILCRAL VA & US & & \\
\hline 20 & $41.009 \angle 4$ & 41.58020 & & & \\
\hline 40 & $41 \cdot 35551$ & 41187753 & 41087734 & & \\
\hline 80 & $\angle 2 n-38 \angle \angle 1$ & 41.38406 & $42 \cdot 33449$ & 41.83461 & \\
\hline 160 & $42 n+35435$ & 41.3432 & $\angle 1 \times 83435$ & $41008<25$ & 41153435 \\
\hline DEPTH & SETTHOS: & .3 & $N 2$ & & \\
\hline 10 & $47+24533$ & TEGSAL UA & ms & & \\
\hline 20 & 47013045 & 571709190 & & & \\
\hline 40 & 47007983 & $27 \times 00304$ & $47 ., 03670$ & & \\
\hline 80 & $\angle 700=6 \angle 3$ & 47009595 & $47 \cdot 09654$ & $47 \cdot 09669$ & \\
\hline 160 & $47+09032$ & $47 \cdot 09628$ & $47 \cdot 09631$ & 47000630 & 47009629 \\
\hline
\end{tabular}

FIGURE B6. Integral values for depth mismatch with a tangential dipole. (Page 5 of 8) 
IEPTH SETTINGS: • 3 • 3

$r$ INTEGRAL VALUES

$1053 \cdot 94770$

$20 \quad 53.79185$

$40 \quad 53 \cdot 74525$

$80 \quad 53+74315$

$160 \quad 53.74303$
$53 \cdot 73990$

$53072973 \quad 53772905$

$53074245 \quad 53 N 74330$

$53074299 \quad 5374308$
53.74353

$53.74303 \quad 53774302$
EEPTH. SETTINGS: $\because 5 \quad$ N4

I INTEGPAL VALTES

$10 \quad 62.75647$

$00 \quad 62454353$

$40 \quad 62 \cdot 47225$

$30.62 \cdot 46908$

$160 \quad 62 \cdot 46322$
$62 \times 47256$

$62 N \angle 4849$

$62 \cdot 46802$

$62 \cdot 46886$

$62 N 44689$

62146932

62.46892
$62 N 46968$

$62 \% 46391$

$$
\begin{array}{ll}
\text { DEPTH } & \text { SETTINGS: } \\
& \\
10 & 74174194 \\
20 & 74.47186 \\
40 & 74.35223 \\
80 & 74.34692 \\
160 & 74.34663
\end{array}
$$

INTEGRAL VAUES

$74 \cdot 38184$

74231236

$74.345: 5$

74.34654

74030772

74.34735

$74 \cdot 34663$
74.34798

$74.3466 \varepsilon \quad 7434662$

$\begin{array}{ll}\text { DEPTH SETTIUGS: } \\ \text { it } \\ 10 & 91 \cdot 65601 \\ 20 & 91.52360 \\ 40 & 91.29304 \\ 80 & 91.23297 \\ 160 & 91 \cdot 23241\end{array}$

NS INTEGRAL VALUES

$$
\begin{aligned}
& 91.47948 \\
& 91.21619 \\
& 91.27962 \\
& 91.28223
\end{aligned}
$$

91.19364

91. 28336

$91 \cdot 28241$

$62 \pi 46391$
DEPTH SETTIUGS:
c.
10115.57997.
$20 \quad 117253708$
$40 \quad 116098511$
$30 \quad 116.26: 75$
$160 \quad 116 \cdot 96049$

N3

IN TECRAS URIUES

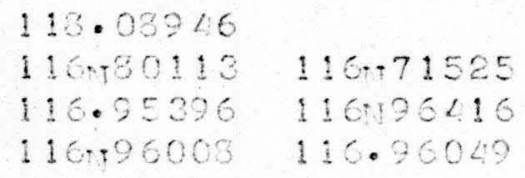

116.96049

116.96811

$116 \times 96043 \quad 1160.96040$

91028528

91.2323391023236

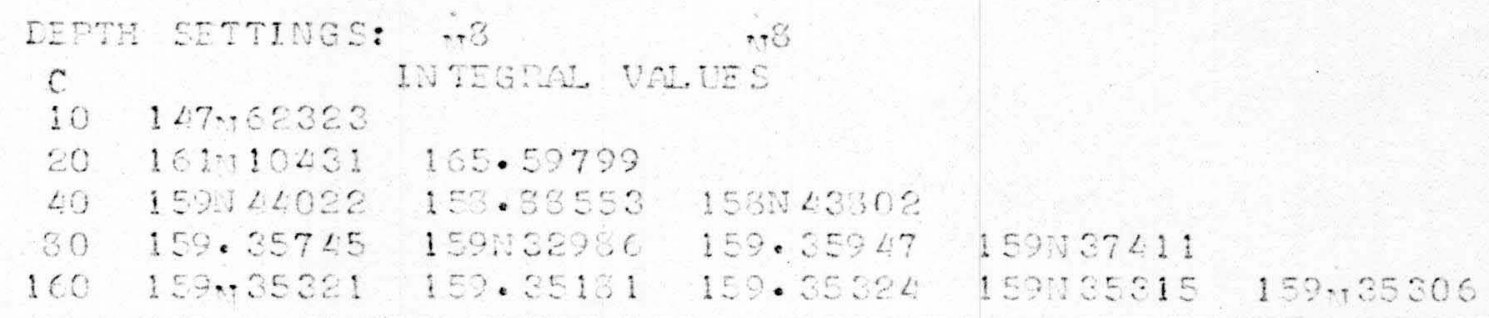

FIGURE B6. Integral values for depth mismatch with a tangentiel dipole. (Page 6 of 8 ) 


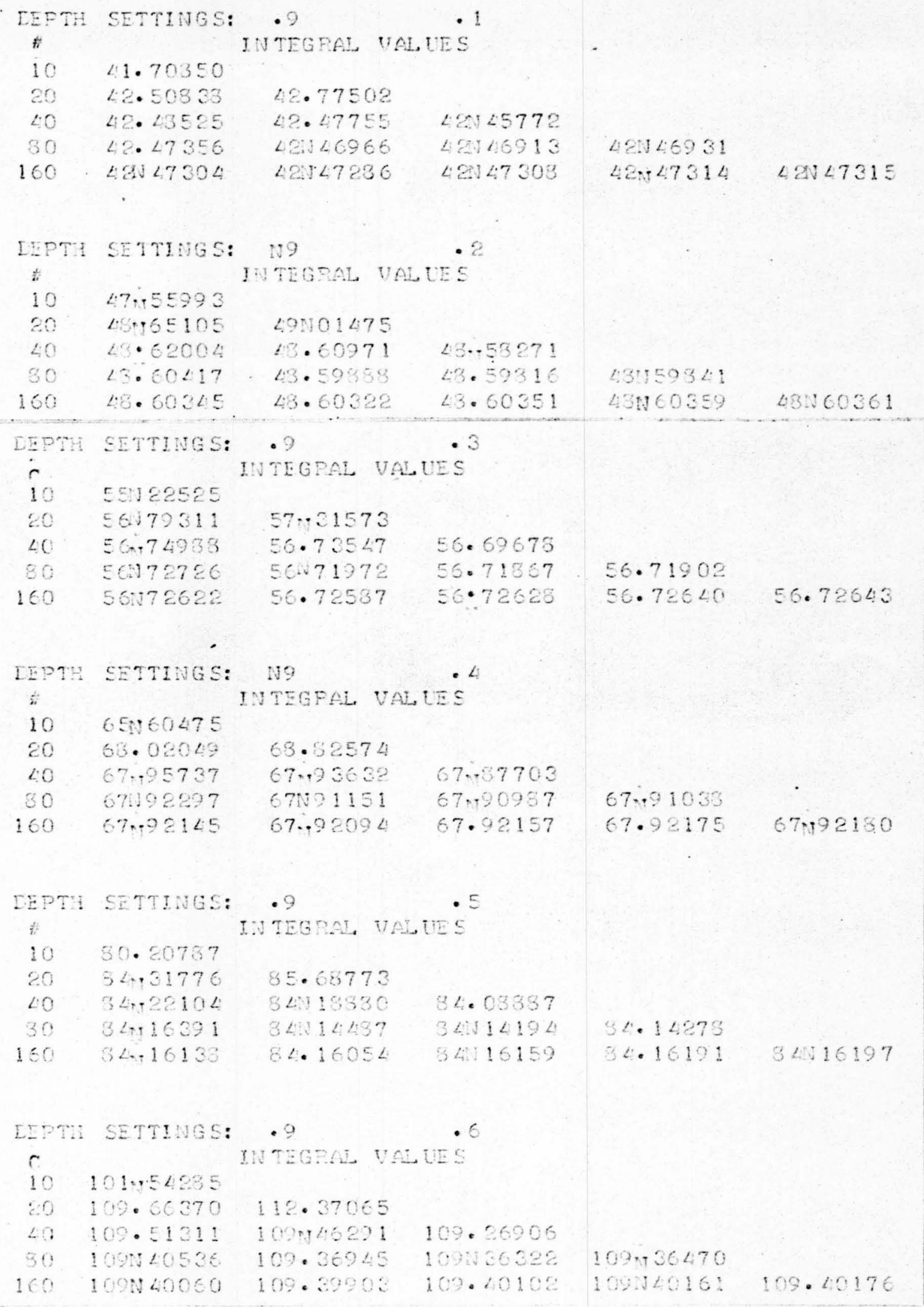

FIGURE B6. Integral values for depth mismatch with a tangential dipole. (Page 7 of 8 ) 


\begin{tabular}{|c|c|c|c|c|c|}
\hline EEPTH & SETTHWGS & .9 & +7 & & \\
\hline 10 & 133.05433 & INTEGFAL VA & UES & & \\
\hline 20 & 153.06970 & $159 \cdot 74148$ & & & \\
\hline 40 & 150.91453 & 152.86233 & $152 v 40430$ & & \\
\hline 30 & 152.66522 & $1520582: 2$ & 15255634 & 152056595 & \\
\hline 160 & $152 \cdot 65420$ & $152 \pi 65054$ & 152465512 & $152 \cdot 65658$ & 152.65695 \\
\hline EEPTI & SETTIUGS: & $\mathrm{Ng}$ & $\cdot 8$ & & \\
\hline $\begin{array}{l}\frac{1}{4} \\
10\end{array}$ & $175 \pi 31088$ & INTEGRAL UA & UE 5 & & \\
\hline 20 & 237082512 & 258166717 & & & \\
\hline 20 & 239.559750 & $240 \cdot 13728$ & $238 N 95529$ & & \\
\hline 30 & 2380755351 & $233 \cdot 47217$ & 238035782 & 238134833 & \\
\hline 160 & 238.71673 & $233 \cdot 70447$ & $208 \cdot 71994$ & 2501372571 & $238 \cdot 72717$ \\
\hline $\begin{array}{l}\text { DEPT: } \\
\#\end{array}$ & SETTINGS: & No & $n 9$ & & \\
\hline 10 & 213.03949 & NTLCRAL VE & UES & & \\
\hline 20 & $412 \cdot 16974$ & 478.54645 & & & \\
\hline 40 & 466013220 & 484011969 & $\angle 32 \angle 9121$ & & \\
\hline 80 & $460+53174$ & 458.66498 & 456.96795 & 456.53106 & \\
\hline 160 & $460 \cdot 20934$ & 460.10254 & $460 \cdot 19336$ & $460 .+24963$ & 460126422 \\
\hline
\end{tabular}

FIGURE B6. Integral values for depth mismatch with a tangential dipole. (Page 8 of 8 ) 
shown explicitly since these parameters are the same for both dipoles and are held constant) and $c_{r}$ is the normallzing constant defined in equation II-28a. Since $R$ has been defined to be unity and the integrand is independent of $\phi$, equation $B 2$ reduces to

$$
b_{r}=\frac{2 \pi}{c_{r}} \int_{0}^{\pi} F_{1}\left(\theta \mid r_{1}\right) F_{1}\left(\theta \mid r_{0}\right) \sin \theta d \theta
$$

Simflarly, for a dipole orlented in the a direction, the integral is

$$
b_{\theta}=\int h_{\theta}(\theta, \phi) v(\theta, \phi) d q
$$

or,

$b_{\theta}=\frac{1}{c_{\theta}} \int_{0}^{2 \pi} \int_{0}^{\pi} F_{2}\left(\theta \mid r_{1}\right)(\cos \phi) F_{2}\left(\theta \mid r_{0}\right)(\cos \phi) R^{2} \sin \theta d \theta d \phi$

Since the integral of $\cos ^{2} \phi$ over $2 \pi$ radians equals $\pi$, this equation reduces to

$$
b_{\theta}=\frac{\pi}{c_{\theta}} \int_{0}^{\pi} F_{2}\left(\theta \mid r_{1}\right) F_{2}\left(\theta \mid r_{0}\right) \sin \theta d \theta
$$

Finally, for a dipole oriented in the $a_{\phi}$ direction, the integral is

$$
b_{\phi}=\int h_{\phi}(\theta, \phi) \mathbf{v}(\theta, \phi) d q
$$

B7

or,

$b_{\phi}=\frac{1}{c_{\phi}} \int_{0}^{2 \pi} \int_{0}^{\pi} F_{2}\left(\theta \mid r_{1}\right)(\sin \phi) F_{2}\left(\theta \mid r_{0}\right)(\sin \phi) R^{2} \sin \theta d \theta d \phi \quad B 8$ 
which simflarly reduces to

$$
b_{\phi}=\frac{\pi}{c_{\phi}} \int_{0}^{\pi} F_{2}\left(\theta \mid r_{1}\right) F_{2}\left(\theta \mid r_{0}\right) \sin \theta d \theta
$$

The program ilsted in $\mathrm{flB}_{\mathrm{B}}$. B4 was written to determine as acceptable way of performing the numerical integrations necessary to evaluate the integrals in equations B3, B6, and B9. Th1s program uses the Romberg integration method. 25 program rosults are shown in figura B5 for a radial dipole and flgure B6 for a tangential dipole (note that the integrals for $\bar{a}_{\theta}$ and $\bar{a}_{\phi}$ directed dipoles are identical). These outputs give tho values of the quantities $b_{r} c_{r}$ and $b_{\theta} c_{\theta}\left(\right.$ or $b_{\phi} c_{\phi}$ ) as functions of $r_{0}$ and $r_{1}$.

From the program outputs, it can be seen that the integral converges rapidis as the number of points used Increases for dipole locations near the center. However, for values of $r_{0}$ and $r_{1}$ greater than approximately 0.7 , convergence is much slower. This result is to be expected since the surface potential curves change more rapidly for dipoles near the surface (see figures III-I and III-2). Based on these results, it was decided that 160 points and a third order approximation should be used in evaluating the integrals (1. …, fifth row, third column, of integral values on the printouts). Another program was written to present the same 
data in more readable form (see figure B7 for listing). The basic changes from the previous program are that the constant $c_{r}$, c ${ }_{\theta}$, or ${ }^{c}{ }_{\phi}$ is included in the computation and that the total signal energy is normalized to unfty. This program therefore calculates the parameter $r$ as defined in equation II-16.

The first step of this progrem is to calculato the integrals of equation $B 3, B 6$, or B9 as follows:

$$
\begin{aligned}
& I_{1}\left(r_{1}, r_{0}\right)=\int_{0}^{\pi} F_{1}\left(\theta \mid r_{1}\right) F_{1}\left(\theta \mid r_{0}\right) \sin \theta d \theta \\
& I_{2}\left(r_{1}, r_{0}\right)=\int_{0}^{\pi} F_{2}\left(\theta \mid r_{1}\right) F_{2}\left(\theta \mid r_{0}\right) \sin \theta d \theta
\end{aligned}
$$

It can be seen that the values of $r_{1}$ and $r_{0}$ may be interchanged without affecting the value of the integral, so these integrals are calculated only for values such that $r_{1}$ is greater than or equal to $r_{0}$.

The program output is as follows. First, for a radial dipole, the integral of the square of the surface potential (the power) is printed for each depth used. Th13 value is equal to the integral $I_{1}$ when $r_{1}=r_{0}$. In addition, the average value of the surface potential is printed. It will be noted that the average is zero to within the accuracy of the computer used (approximately six decimal digits). Next, the values of the parameter $\gamma$ are printed for values of $r_{0}$ and $r_{1}$ from 0.1 to 0.9 in 0.1 steps. The value of $\gamma$ is calculated as 
WOOD FEM FILTER FESFONSE FOR DEFTH MISMATEH.

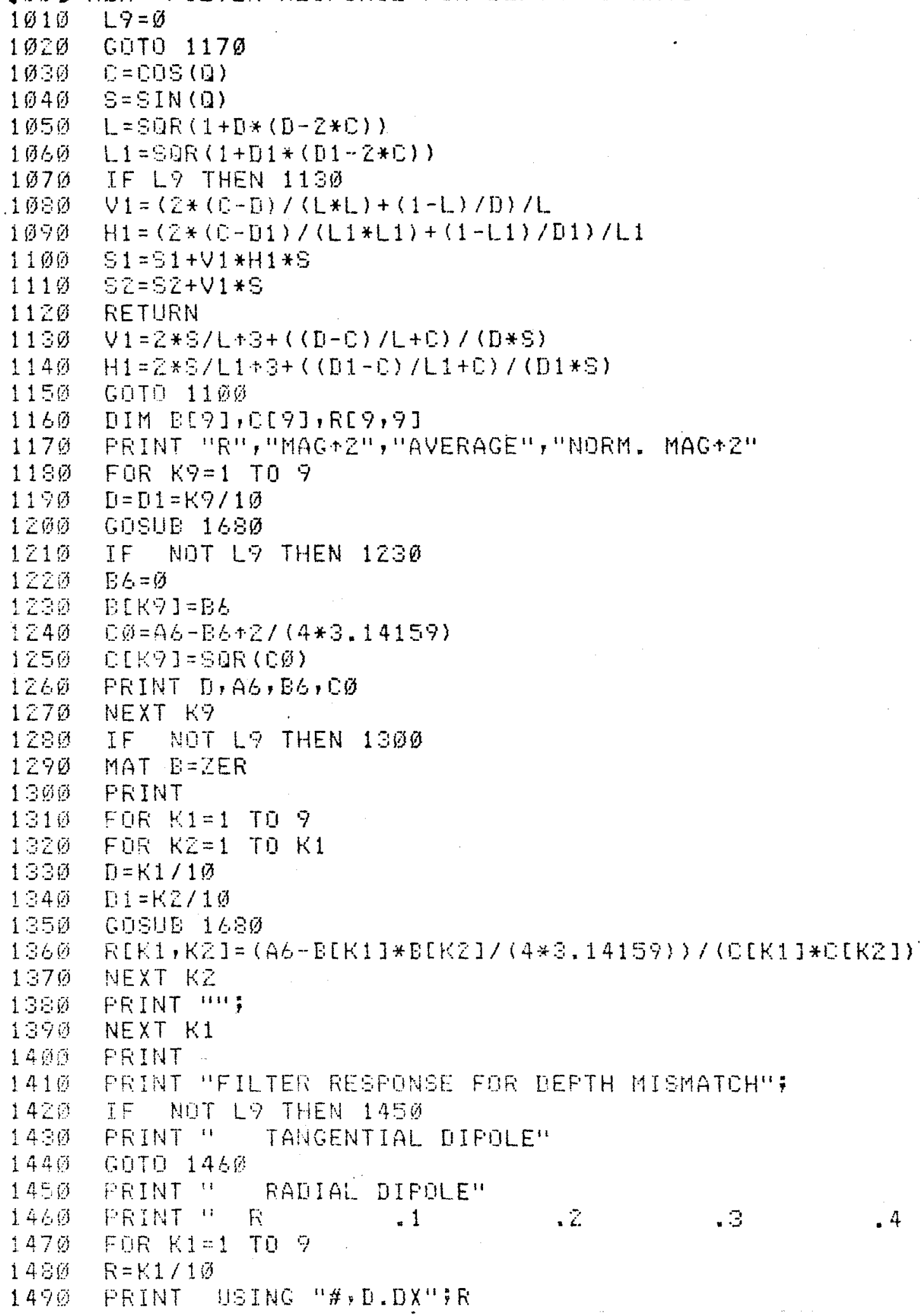

FIGURE B7. Final program to ovaluate filter response for depth mismatch. (Pago 1 of 2) 


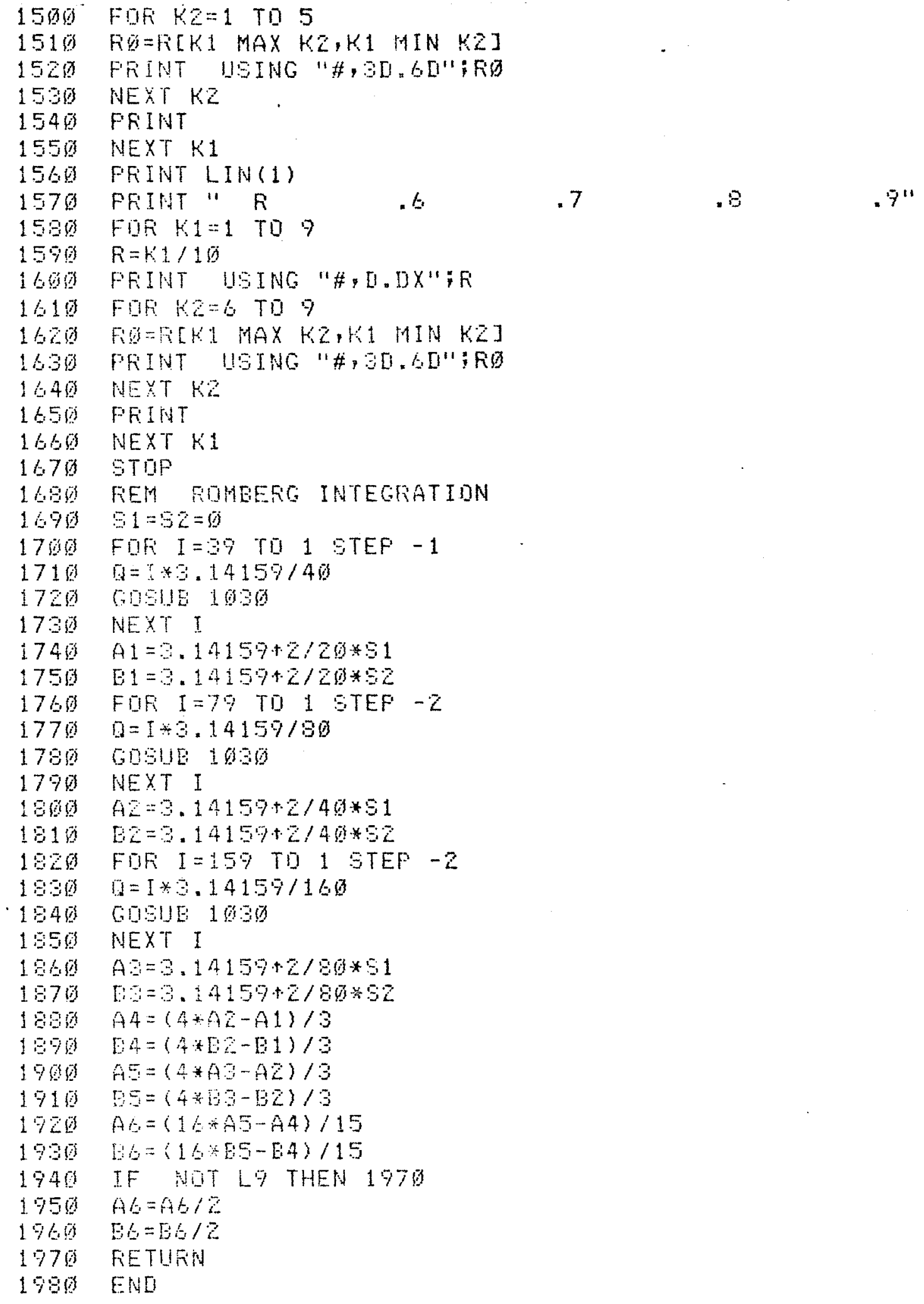

FIGURE B7. Final program to evaluate filter response for depth mismatch. (Page 2 of 2 ) 

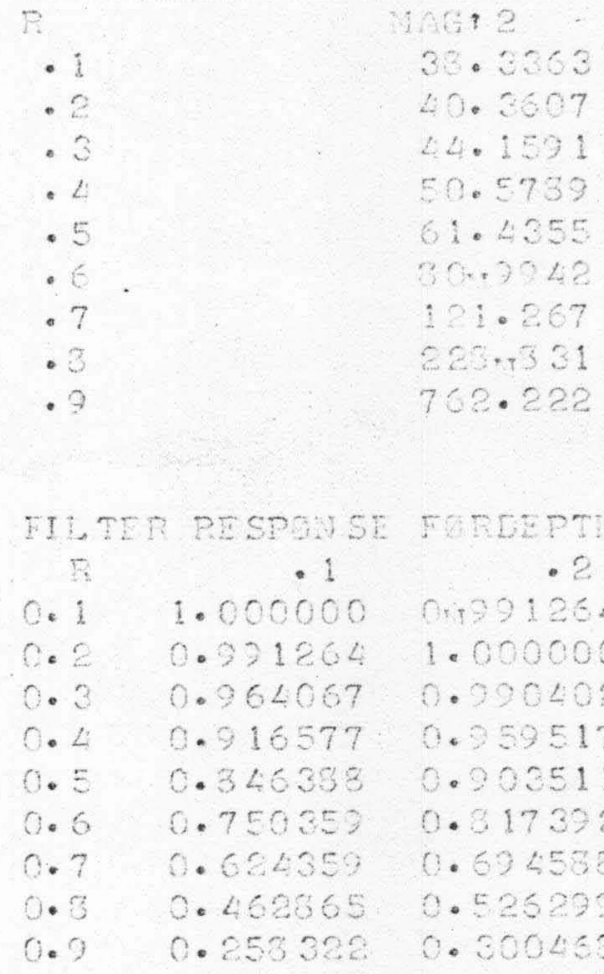

TOLEPTH MI SHATOI
.2

RADIAL DIPOLE

$\begin{array}{cc}04 & .5 \\ 0.916577 & 0.346333 \\ 0.959517 & 0.903511 \\ 0.983376 & 0.051693 \\ 1.000000 & 0.236264 \\ 0.956264 & 1.0000000 \\ 0.938165 & 0.931614 \\ 0.841894 & 0.913161 \\ 0.676994 & 0.765444 \\ 0.412490 & 0.439506\end{array}$

\begin{tabular}{|c|c|c|c|c|}
\hline ? & . 6 & .7 & .8 & .9 \\
\hline 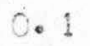 & 0.750353 & $0.62 \angle 359$ & 0.462865 & 0.253322 \\
\hline & 0.817392 & 0.534588 & 0.526299 & 0.300463 \\
\hline & 0.831241 & 0.767676 & 0.597344 & $0 .:$ \\
\hline & 0.933166 & 0.541394 & 0.676994 & 0.412490 \\
\hline & 0.381614 & 0.213161 & $0.765 \angle 2 \angle$ & 0.439506 \\
\hline & 1.000000 & 0.972267 & 0.360240 & $0.5379:$ \\
\hline & 0.2722667 & $1 \cdot 060000$ & 0.950716 & 715519 \\
\hline & $0.3602 \angle 0$ & 0.250746 & $i \cdot 000000$ & 749 \\
\hline$\cdots$ & 0.587255 & 0.745512 & 0.874273 & 10000000 \\
\hline
\end{tabular}

FIGURE B8. Computed radial filter response for depth mismatch. The simulated source is a radial dipole. 


$P$
$\cdot 1$
.2
$: 3$
$: 4$
15
.6
.7
.8
.9

\section{MAS 12}

33.1763

39.6526

42.47288

47.0962

52.7031

67.2211

930077

159.353

$450 \cdot 197$

AVERAGE
0
0
0
0
0
0
0
0
0

$$
\begin{aligned}
& \text { NOW. MAGT2 } \\
& 33 \cdot 1763 \\
& 39 \cdot 6326 \\
& 42.4723 \\
& 4710962 \\
& 54.7031 \\
& 67.9211 \\
& 93.9077 \\
& 159.353 \\
& 460.197
\end{aligned}
$$

ILTEP RESPONSE FOREEPTH MI STATCH

$=$

C11 11.000000

$0.2 \quad 0.993409$

as 0.272639

$0.4 \quad 0.935350$

$0.5 \quad 0.379924$

0.60 .300334

0.7

0.8

0.9

0.1

0.2

0.3

0.4

O.. 5

0.6

0.7

0.3

0.9
0.689960

0.537000

0.320438
.2

0.923409

1. 000000

0.992692

0. .65552

0.324533

0.354078

$0.74326 ?$

0.592251

0.359662
.3

0.272682

0.992592

1.000000

0.291407

0.352133

0.204214

0.808202

0.653259

0,405747
TANGENTIAL, DIPOLE

$0 \cdot 035 \% 50$

0.879924

$0.968352 \quad 0.924538$

0.2314070 .962133

1.0000000 .252159

$0.989159 \quad 1.000000$

0N950223 0.235051

$0.3620110 .927<31$

$0.721090 \quad 0.796205$

$0.461368 \quad 0.530437$
0.800334
.7
0.557000
C.... .9
0.682960
0.320433
0.854073
0.743262
0.522251
0.359662
0.904914
0.308302
0.653259
0.405747
0.950223
0.869011
0.721020
0.461362
0.985051
0.727481
0.796275
0.377412
0.976702
0.976702
1. 000000
0.255109
0.530437
0.618793
0.734324
0.377412
0.956109
1. 000000
04331583
0.618793
0.734324
0.381523
110000000

FTGURE B9. Corputed tangential filter response for depth mismatch. The simulated source is a tangential dipole. 


$$
r_{1}=I_{1}\left(r_{1}, r_{0}\right) /\left[I_{1}\left(r_{1}, r_{1}\right) I_{1}\left(r_{0}, r_{0}\right)\right]^{\frac{1}{2}} \quad \text { B12 }
$$

Output for a tangential dipole is similar. In thls case, the average value of the surface potential is set to zero by the program. The average value 18 zero in this case because of the sinusoldal variation of the potential with. The parameter $\gamma$ is bero calculated as

$$
r_{2}=I_{2}\left(r_{1}, r_{0}\right) /\left[I_{2}\left(r_{1}, r_{1}\right) I_{2}\left(r_{0}, r_{0}\right)\right]^{\frac{1}{2}}
$$

Outputs for radial and tangential dipoles are listed in figures B8 and B9. These data ore also plotted in figure III -4 .

\section{POSITION VARIATION SIMULATION}

A fourth program was written to evaluate the filter output for the case in which the actual dipolo and the filter dipole locations are independent. This program is required to perform a two dimensional numerical integration. The listing is shown in $\mathrm{fig} . \mathrm{BlO}$ and the results are shown in figure Bll.

For this program, the coordinate system is defined such that the actual dipole lies on the positive $z$ axis. The filter dipole may be located anywere within the sphere. Parameters which must be specified include the actual dipole orientation and depth and the filter dipole orien- 


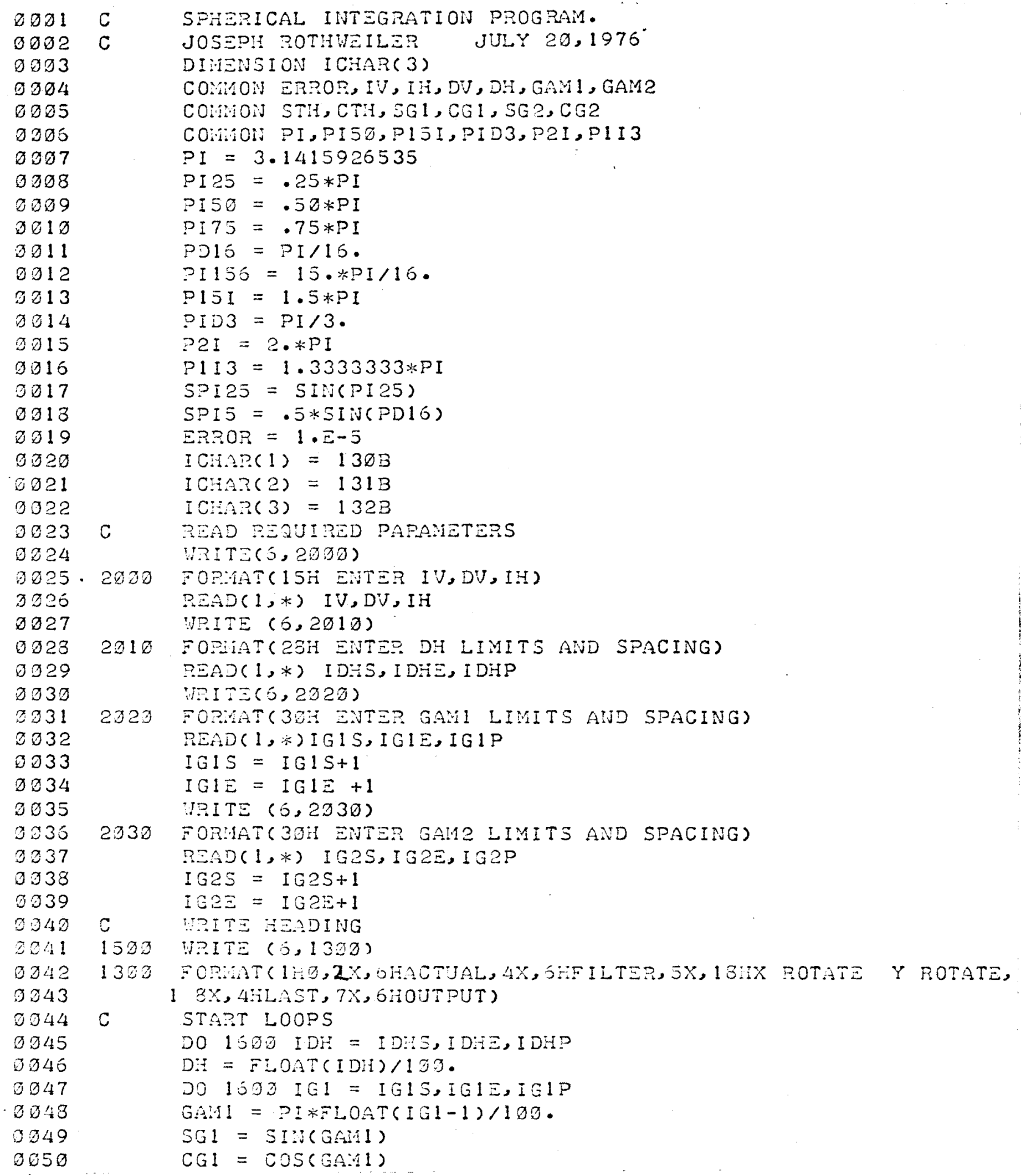

FIGURE B10. Program to calculate filter response for general position mismatch. (Page 1 of 4 ) 


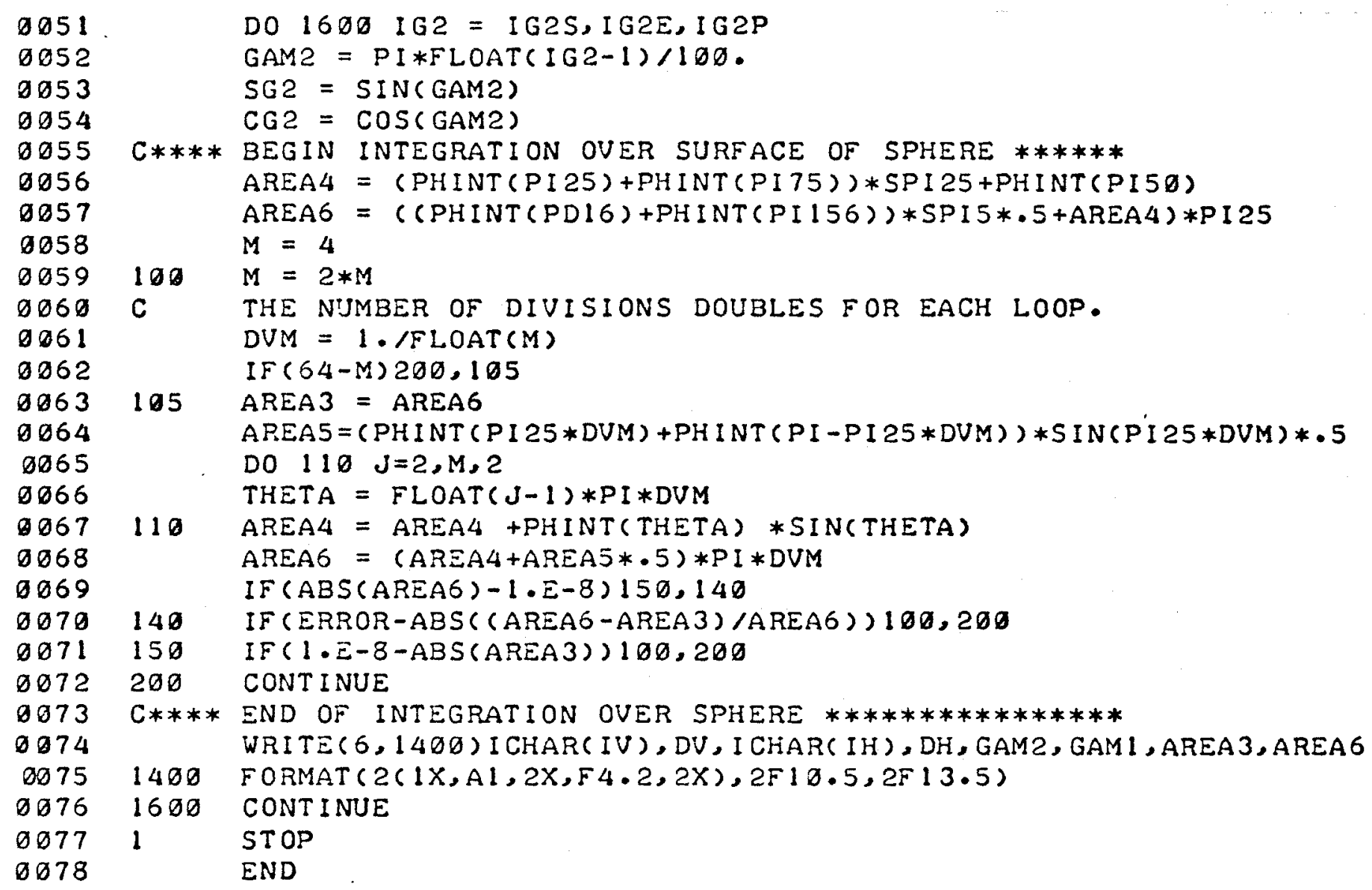

FIGURE BI0. Program to calculate filter response for general position mismatch. (Page 2 of 4 ) 


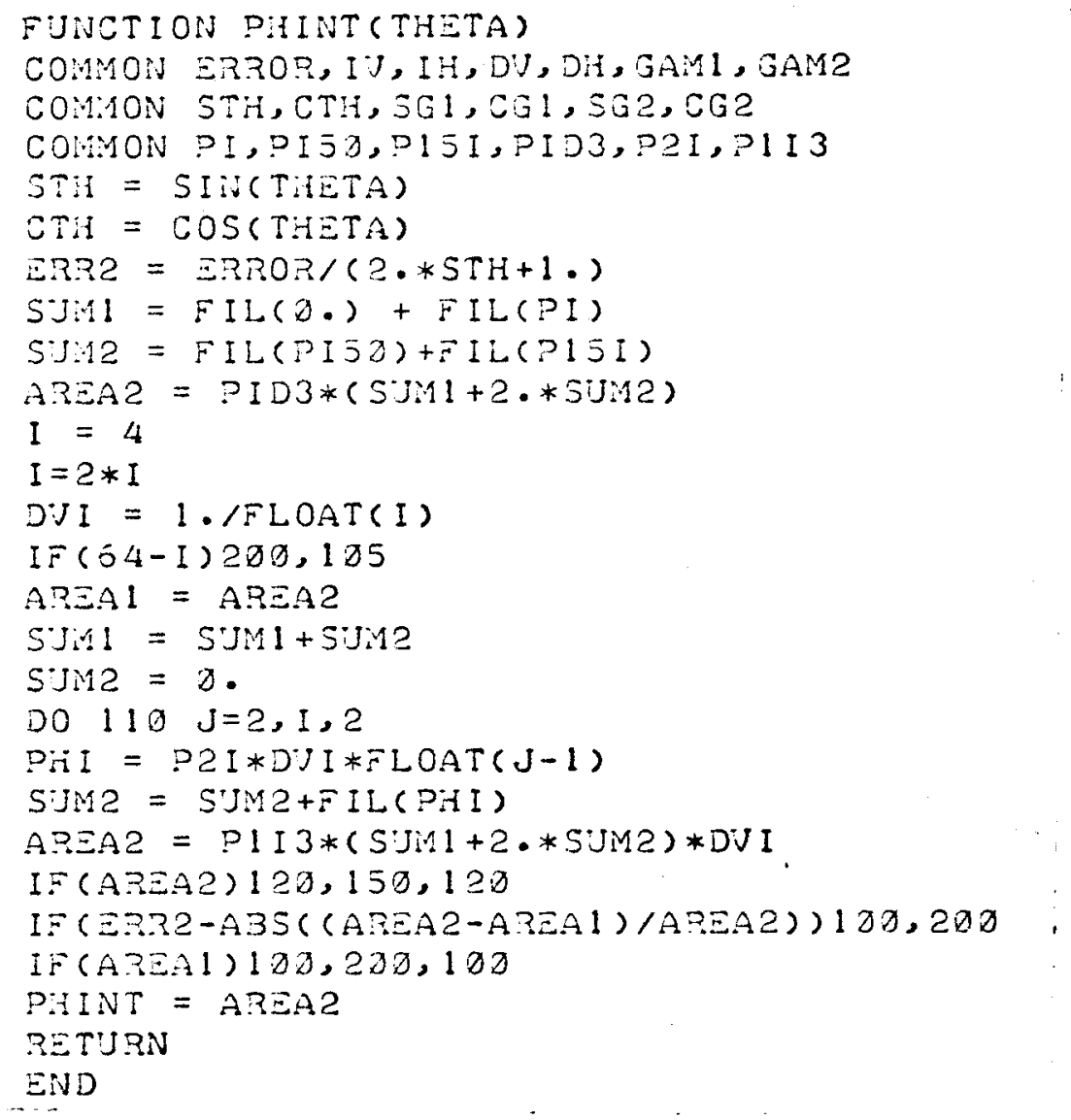

FIGURE B10. Program to calculate filter response for general position mismatch. (Fage 3 of 4 ) 


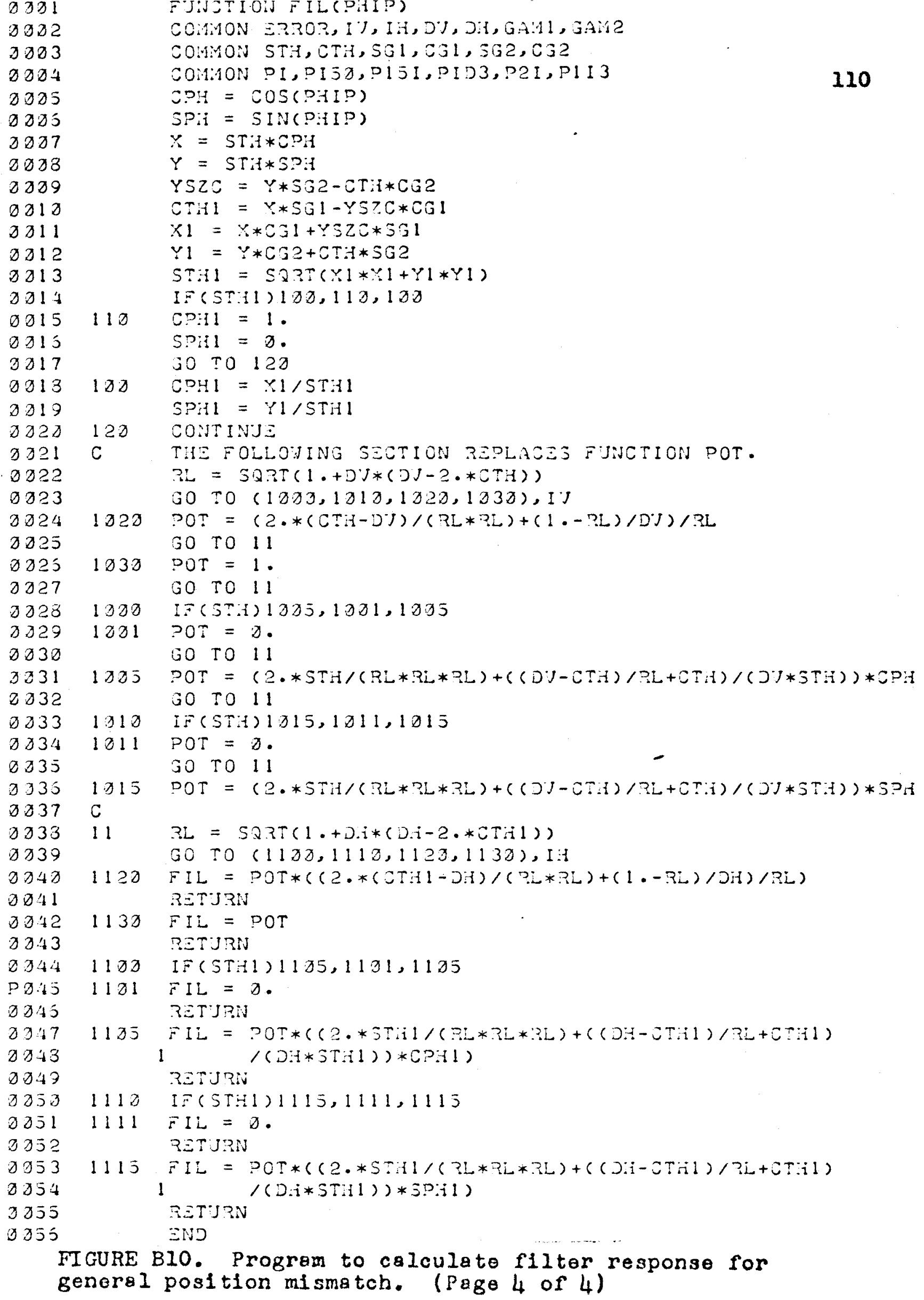

2019

$D D 2 D$

ว $ว 21$

0 022

ด $\geq 3$

ว 024

ว 025

๑ว25

ว 27

Dว 28

D 829

จ 30

3331

乙 ग 32

D 33

D 34

0035

0336

ว 37

D 33

ว 39

0248

0041

$00 \div 2$

ว 343

0344

$P 0.45$

0045

ว 047

2043

0049

0253

ว 01

3 $\supset 5 ?$

ว 953

D254

3255

ด 355

FIGURE B10. Program to calculato filter response for general position mismatch. (Page 4 of 4 )

COMMON E3ROR, I\%, IH, DJ, JIT,GAH1, GAMR

COMMON STH, CTH, SG1, G31, 3G2, C32

COHMON PI, PISD, PISI, PID3, P2I, PII3

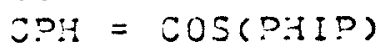

$S P_{i I}=\operatorname{SIN}\left(P_{I I I P)}\right.$

$X=$ STiA $*$ CPH

$Y=S T i A * 5 P: I$

YSZS $=$ Y*SG2-CT:I*CG2

CTHI $=$ Y*SGI-YSTC*CGI

$x 1=x * 0 a 1+Y \leq 20 * 531$

$Y 1=Y * C G 2+C T A * 5 G 2$

$S T: I 1=S Q T T(Y) * Y 1+Y 1 * Y 1)$

$1 F(5 T 11) 100,110,100$

$110 \quad C P: 11=1$.

SP:il $=D$.

SO TO 120

$1 D D$ CDH $=X 1 / 5 T H 1$

$S P: H 1=Y 1 / S T H 1$

120 CONTINJE

C THE FOLLOJING SECTION REPLACEJ FJINCTION POT.

$B L=3 Q 3 T(1 \cdot+D J *(D J-2 \cdot * T T H))$

GO TO $(1 \partial \partial 3,1010,1020,133 \partial), 11$

$1 D 2 D$ POT $=(2 \cdot *(C T H-D J) /(R L * P L)+(1 .-P L) / D J) / B L$

GO TO 11

$103 \partial$ POT $=1$.

GO TO 11

$10 \partial 0$ IF(5T:1)1305,1001,1005

$1 \partial \partial 1$ POT $=D$.

GO TO 11

$1 \partial \partial J P O T=(2 . * 5 T H /(R L * R L * P L)+((D)-C T H) / P L+C T H) /(J) * S T H)) * C P H$ GO TO 11

1010 IF(STA)1015,1011,1015

1011 POT $=0$.

SO TO 11

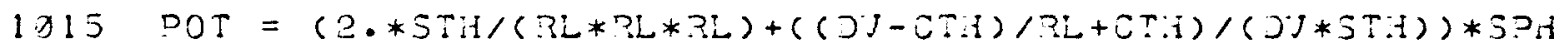

C

$11 \quad 3 L=\operatorname{SQRT}(1 \cdot+D \cdot i *(D i-2 \cdot * 0 T i i 1))$

GO TO $(1100,1113,1123,1130)$, I:

$1120 \mathrm{FIL}=\mathrm{POT} *(2 \cdot(2 *(\mathrm{TTH} 1-\mathrm{DH}) /(\mathrm{PL} * \mathrm{RL})+(1 \cdot-\mathrm{RL}) / \mathrm{DH}) / \mathrm{RL})$

RETJRN

1133 FIL $=$ POT

reTURT

$11 D D$ IF(STH1)11D5,1101,11D5

$1101 F I L=D$.

RETJPN

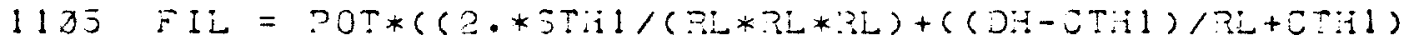

$1 /(D H * 3 T$ T 1$) *(P A 1)$

RETURiv

1110 IF(5TH1)1115,1111,1113

$1111 F I L=0$.

RETJRN

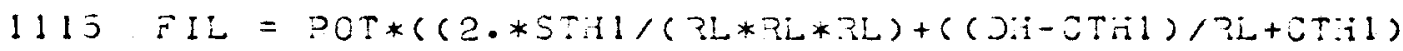

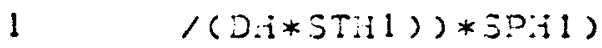

BETURN

END 


\begin{tabular}{llll} 
ACTUAL & \multicolumn{2}{c}{ FILTER } \\
$Y$ & .50 & $Z$ & .50 \\
$Y$ & .50 & $Z$ & .50 \\
$Y$ & .60 & $Z$ & .50 \\
$Y$ & .60 & $Z$ & .50 \\
$Y$ & .50 & $Z$ & .50 \\
$Y$ & .60 & $Z$ & .60
\end{tabular}

$\begin{array}{cc}\text { X.RTATE } & \text { Y ROTATE } \\ .00300 & .00000 \\ .31415 & .00000 \\ .52332 & .03730 \\ .94248 & .00000 \\ 1.25554 & .00000 \\ 1.57030 & .00000\end{array}$

$X$ RotaTE $Y$ Rotate .00030

.20330

- 00000

.00080

.00300

.33302

$X$ ROTATE

. $0007 \varnothing$

.31416

.62332

.94248

1.25554

1.57382

.00000

.31416

.62332

.94243

1.25564

1.57230

.23308

.31415

.52332

.94248

1.25564

1.57380

- 01080

.31415

. 2332

.94248

1.25654

1.57030

. 20000

.31415

. 52332

.94243

1.25554

1.57230
- 3000z

.31415

. 62832

.94243

1. 25564

1.57030

Y ROTATE

- DDDDD

.00080

.00202

.30003

- 00200

- 23200

.00033

- 00020

- $D 0000$

.02022

- 20200

- 00000

- 0003

.00302

- 20302

- 02333

. 00000

.00000

- 30300

.23202

.02030

- DaDor

- 00920

.23302

.00330

- 23000

.03330

- 00302

- $0023 \mathrm{a}$

.32002

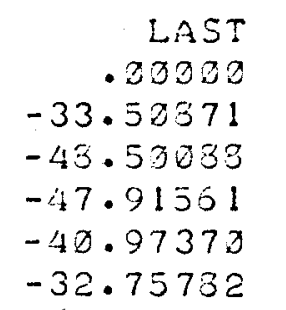

LA่sT

.20000

-. 00000

-.00001

$-.02001$

$-.03000$

$-.32202$

LAST

52.91133

43.22216

35.17762

21.38579

5.50571

$-5.43933$

5. 31775

53.74205

37.38554

19.94759

4.55198

$-7.31392$

69.61311

$5 x .22122$

39.31763

13.35228

2.40342

$-8.42299$

31.53995

67.30545

40.27853

15.23593

.24350

$-9.53235$

97.27093

75.59773

40.52537

13.77015

$-1.87343$

$-10.54139$
OUTPUT

-.02323

$-33.53447$

$-48.49399$

$-47.91491$

$-40.97340$

$-32.75758$

OJTएग

$-.00000$

-.02000

-.00031

$-.0 \partial 000$

-. $0 \partial \partial \partial 0$

- $D D D D D$

OUTPUT

52.75607

43.08551

35.03723

21.04243

5.59705

$-5.42252$

53.11652.

53.57832

37.79374

19.91149

4.55899

$-5.99 \times 55$

69.33854

57.22132

39.22755

18.32653

2.41482

$-8.42023$

31.13512

57.53229

43.19913

15.27257 .25621

$-9.52519$

95.31551

75.41437

40.45732

13.75912

$-1.85573$

$-10.51587$

FIGURE BII. variation.

Simulation results for general position 
tation. In addition the range of values for the filter dipole location parameters (variables DH, GAMI, and GAM2 in the program) are specifled. Referring to fig. Blo, lines 55 through 72 perform the integration over $\theta$. This integration 18 performed using a rectangular integretion method, as diagramed in fig. B12. Inftially, Iines 56 and 57 calculate the integral using four points. Lines 59 through 71 form a loop which evaluates the integral for succossively larger numbers of points, M. Varlablo AREA6 is the new value of the integral, AREA 3 is the value from the previous iteration, AREAL is the sum of the values of all points calculated (so that points from previous iterations need not be recalculated), and AREA5 is an additional factor due to the two end points shown In figure B12. The loop terminates when any one of the following conditions is met:

(1) The integral is less than $10^{-8}$ on two successive passes.

(2) The fractional change in the value of the integral between two passes is less than the variable named ERROR.

(3) The integral has been evaluated for 64 points. The function PHINT is called by the main program to perform the integration over $\not$. This integral is evaluated using SImpson's method. Variables SUMI and 


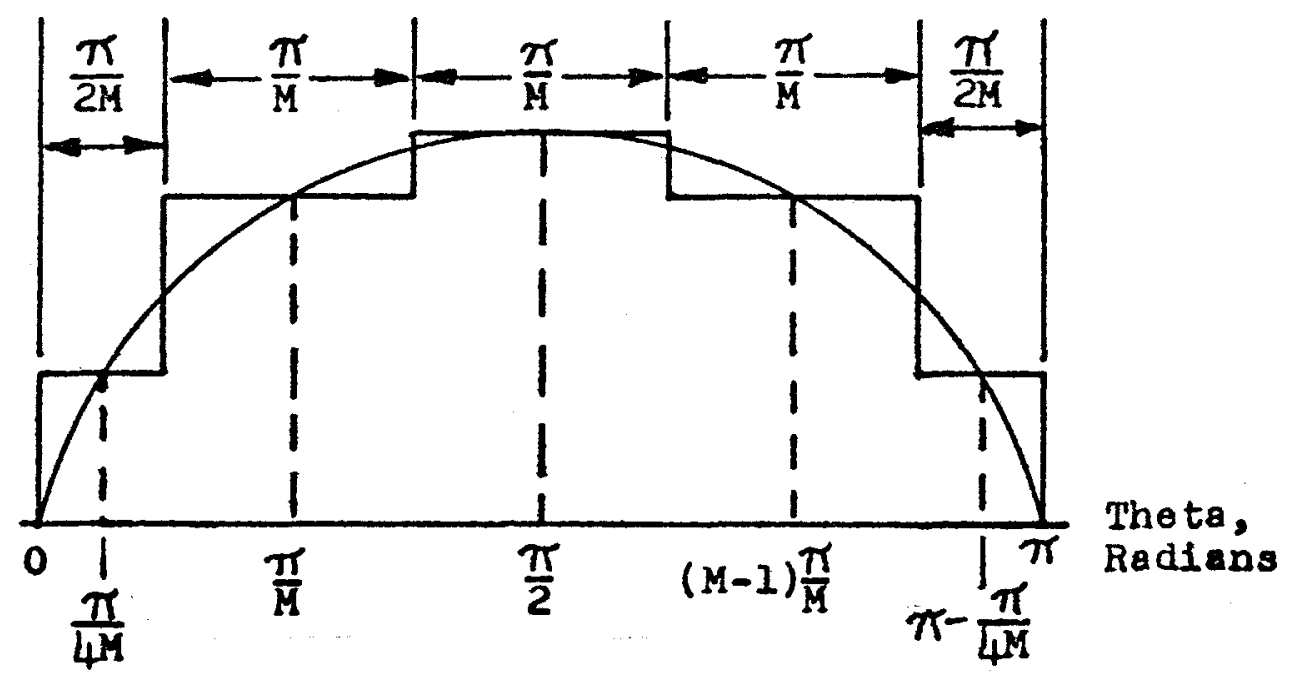

FIGURE B12. Rectangular integration method used for integration over theta. Tho diagram is drawn for $M=4$. 
SUM2 hold the totals of the calculated values for even and odd points, respectively. Variable AREA2 is the latest value of the integral, whlle AREAl is the previous value. Criteria for terminating this integration are the same as for the $\theta$ integration, except that ERR2 rather than ERROR is the variable used in the second test.

Function FIL is called by PHINT to evaluate the product of the surface potential and the filter response function at a given polnt. The surface potential and filter response are both calculated using equations A16 through A18. Since these equations assume that the dipole lies on the $z$ axis, a coordinate transformation must be performod to evaluate the fllter response. This transformation may be devoloped in two steps. First, rotate the coordinate system about the $x$ axis through an anglo* $\gamma_{2}$, producing the coordinate $\left(x^{n}, y^{n}, z^{n}\right)$. Second, rotate the new coordinate system about the $y^{\prime \prime}$ axis through an angle $\gamma_{1}$, producing the coordinate system $\left(x^{\prime}, y^{\prime}, z^{\prime}\right)$. Given a polnt with coordinates $(x, y, z)$, the coordinates after the first transformation are 26

$$
\begin{array}{ll}
x^{\prime \prime}=x & \text { B14a } \\
y^{\prime \prime}=y \cos \gamma_{2}+z \sin \gamma_{2} & \text { B14b } \\
z^{n}=-y \sin \gamma_{2}+z \cos \gamma_{2} & \text { B14c }
\end{array}
$$

* Note that $\gamma_{1}$ and $\gamma_{2}$ are not related to the function $\gamma$ dofined in chapter II. 
The second transformation produces

$$
\begin{array}{ll}
x^{\prime}=-z^{\prime \prime} \sin \gamma_{1}+x^{n} \cos \gamma_{1} & \text { B15a } \\
y^{\prime}=y^{\prime \prime} & \text { B15b } \\
z^{\prime}=z^{n} \cos \gamma_{2}+x^{n} \sin \gamma_{2} & \text { B15c }
\end{array}
$$

or, comblining the two sets of equations,

$$
\begin{array}{ll}
x^{\prime}=x \cos \gamma_{1}+y \sin \gamma_{1} \sin \gamma_{2}-z \sin \gamma_{1} \cos \gamma_{2} & \text { Bl6a } \\
y^{\prime}=y \cos \gamma_{2}+z \sin \gamma_{2} & \text { B16b } \\
z^{\prime}=x \sin \gamma_{1}-y \cos \gamma_{1} \sin \gamma_{2}+z \cos \gamma_{1} \cos \gamma_{2} & \text { Bl6c }
\end{array}
$$

In function FIL, the coordinates of the surface polnt at which the potentials are to be evaluated are given by the variables $\mathrm{CTH}$ and STH, the cosine and sine of $\theta$, and PHIP, the $\phi$ coordinate. These are then changed to the reotangular coordinates $x, y, z$ represented by variables $X, Y$; and CTH. New coordinates $x^{\prime}, y^{\prime}, z^{\prime}$ are then calculated (varlables XI, YI, and CTHI). Finally, the position variables STH $l, C P H I$, and SPHI, the sine of $\theta^{\prime}$ and the cosine and sine of $\phi^{\prime}$, respectively, for the now coordinate system aro calculated.

The surface potential is calculated assuming the dipole is on the positive $z$ axis. The filter response Is calculated for a dipole on the $z^{\prime}$ axis.

Outputs of this program are shown in figure Bll. The printout format is as follows. First, the direction $(x, y$, or $z)$ and radial position of the actual dipole are printed. Second, the direction $\left(x^{\prime}, y^{\prime}\right.$, or $\left.z^{\prime}\right)$ and radial 
position of the dipole to which the filter is matched are printed. Third, the value of $\gamma_{2}$ in radians is printed (laboled $X$ ROTATE). Fourth, $\gamma_{2}$ is printed (labeled Y ROTATE). Fifth, the integral approximation obtained in the next to last iteration of the integration over $\theta$ is printed. Sixth, the final integral epproximation is printed (labeled OUTPUT).

The value from the next to last integration is printed as a check on the convergence of the integration algorlthm. Comparison of this value with the final value, and comparison with the results of the previous program, for depth mismatch, Indicate the accuracg of this program to be approximately three significant flgures. Execution time was found to be 1 to 2 minutes per integration on a Hewlott-Packard 2IMX computer.

SIMULATION FOR DIFFERENT NUMBERS OF ELECTRODES

A program was written in Basic to simulate the filter for a finite number of electrodes. This program consists of a main program and six subroutines (seo fig. B13). The sterting line numbers and functions of the subroutines are as follows:

(1) Line 5100. This subroutino calculatos the 


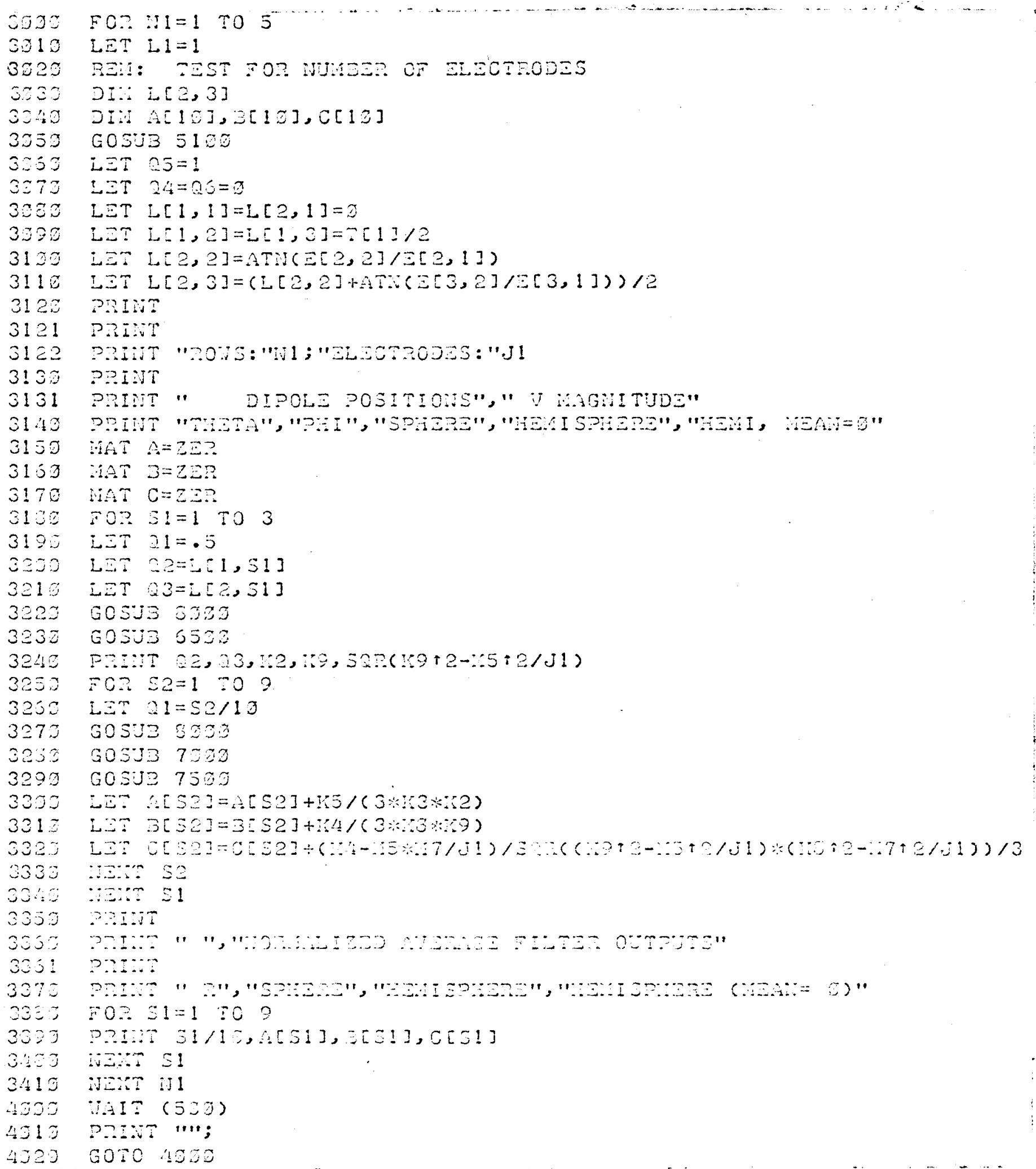

\section{FIGURE BI3. Simulation program for the discrete case.} (Page 1 of 5 ) 


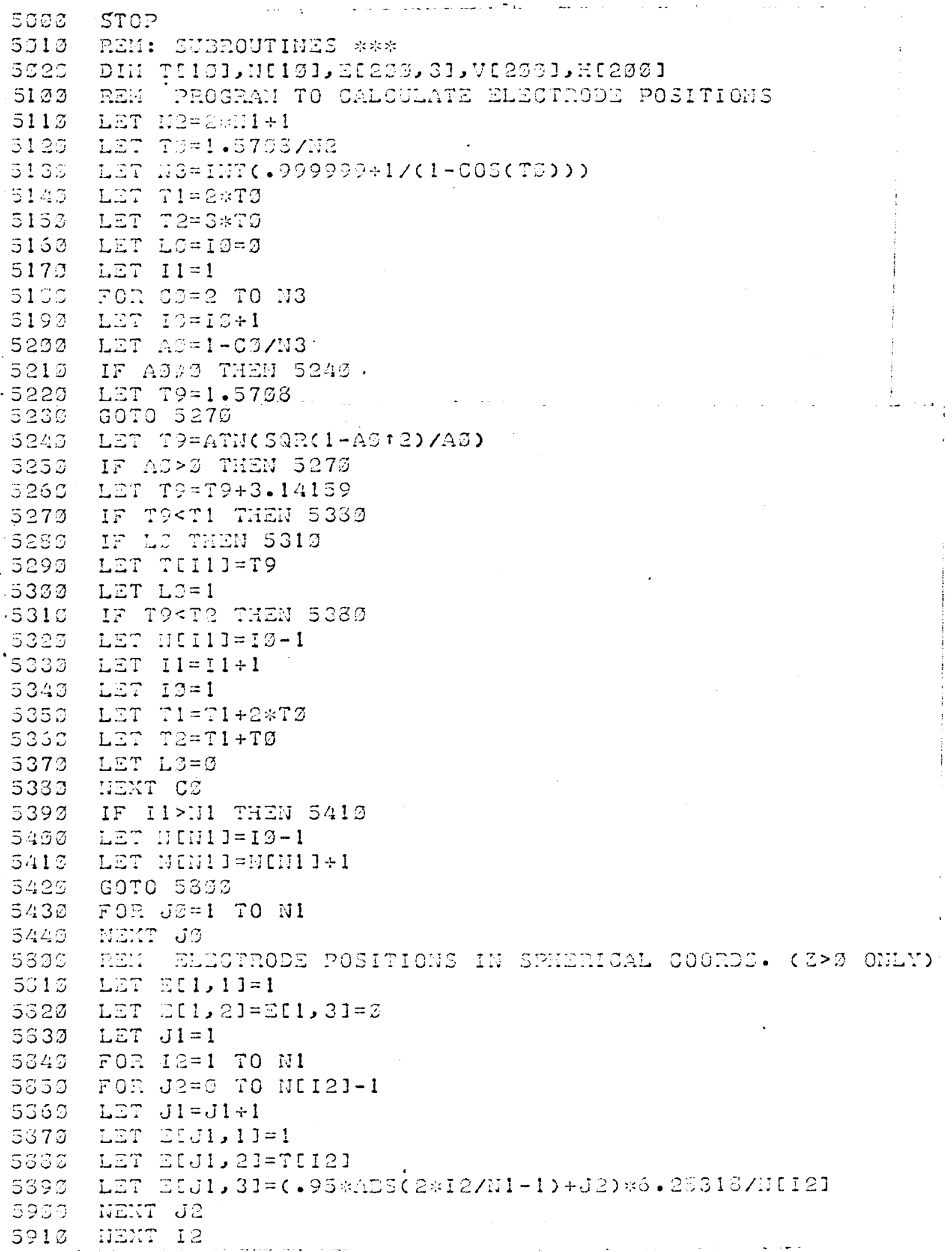

FIGURE BI3. Simulation program for the discrete case. (Page 2 of 5 ) 


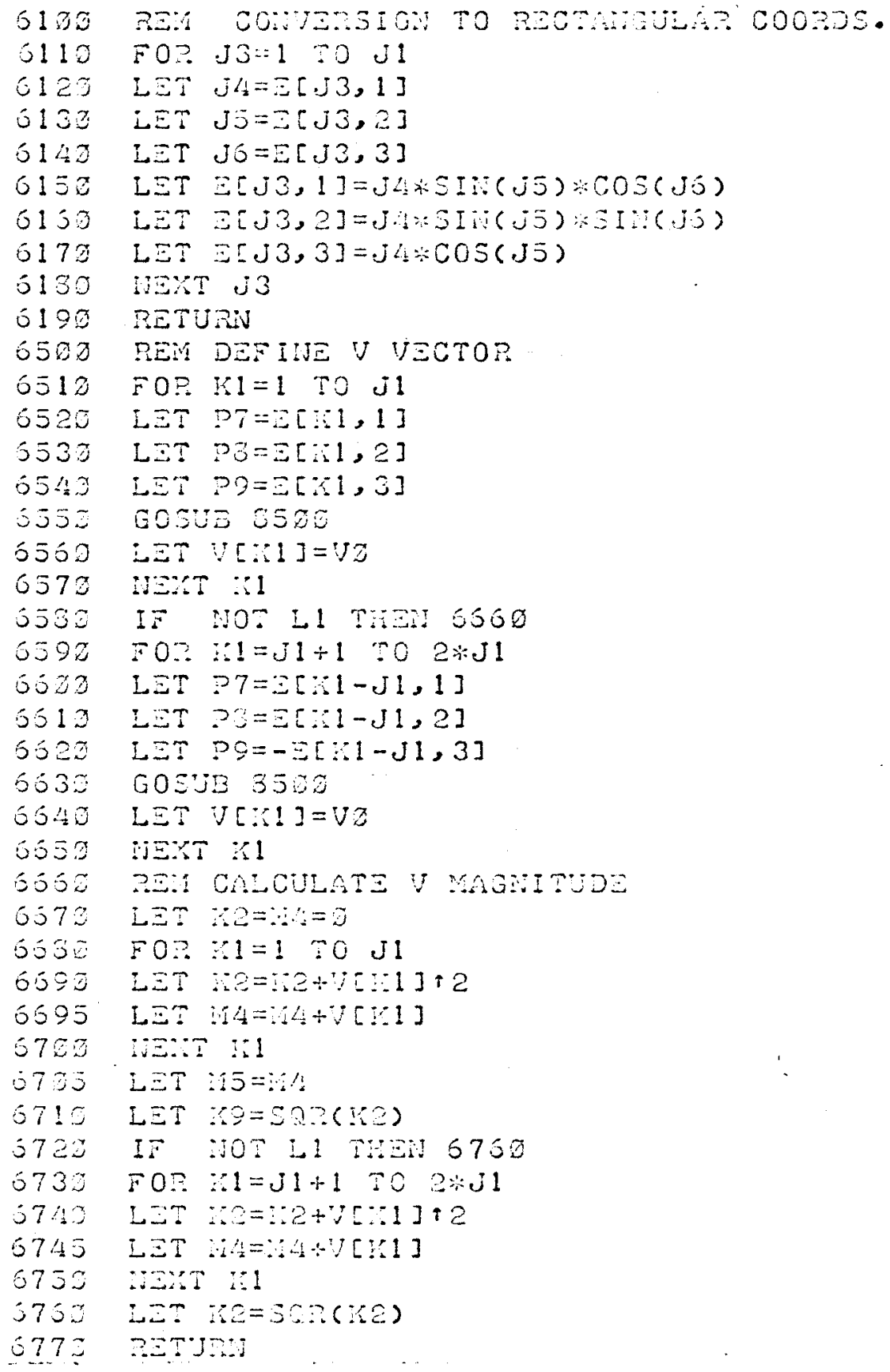

FIGURE B13. Simulation program for the discrete case. (Page 3 of 5 ) 


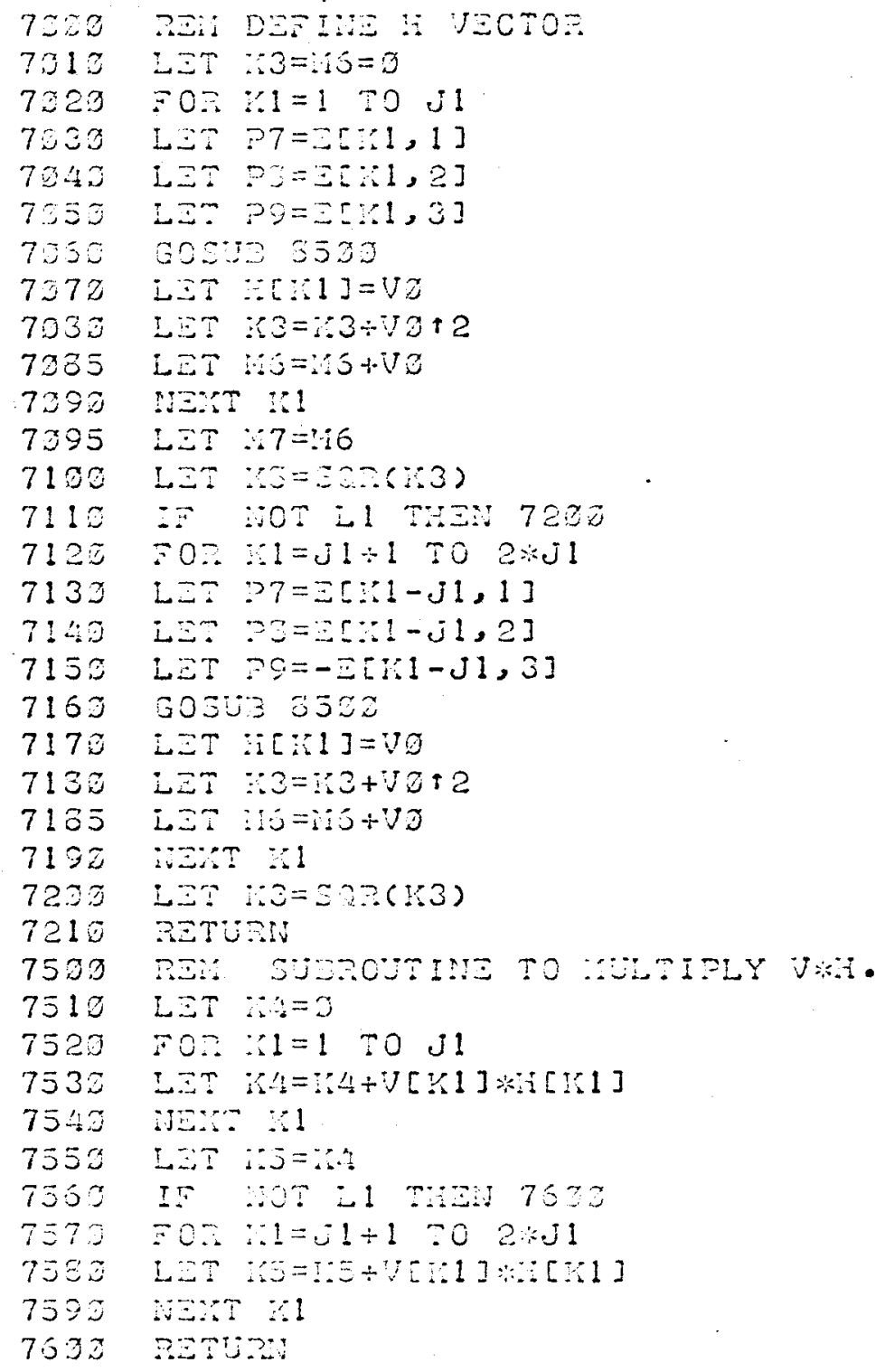




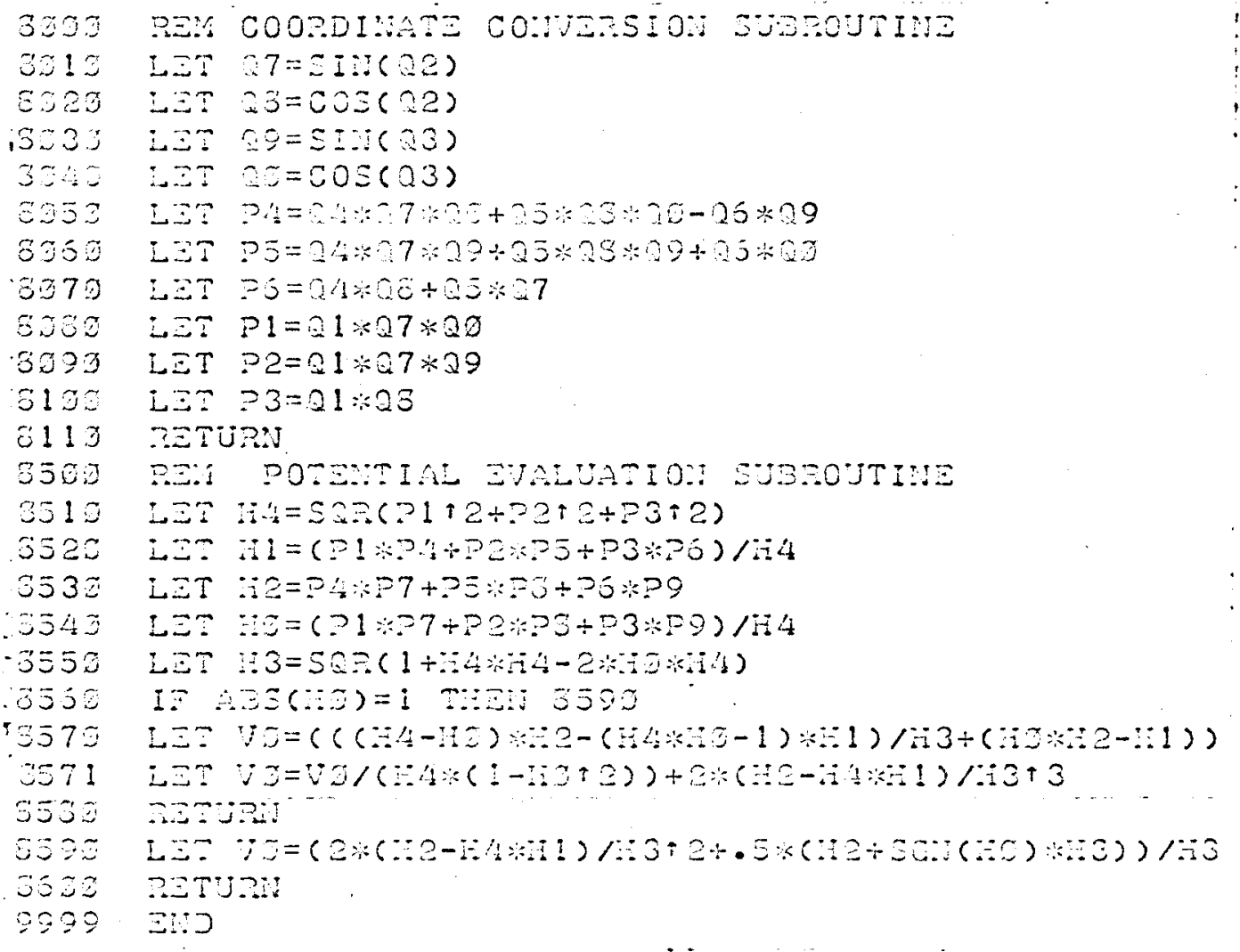




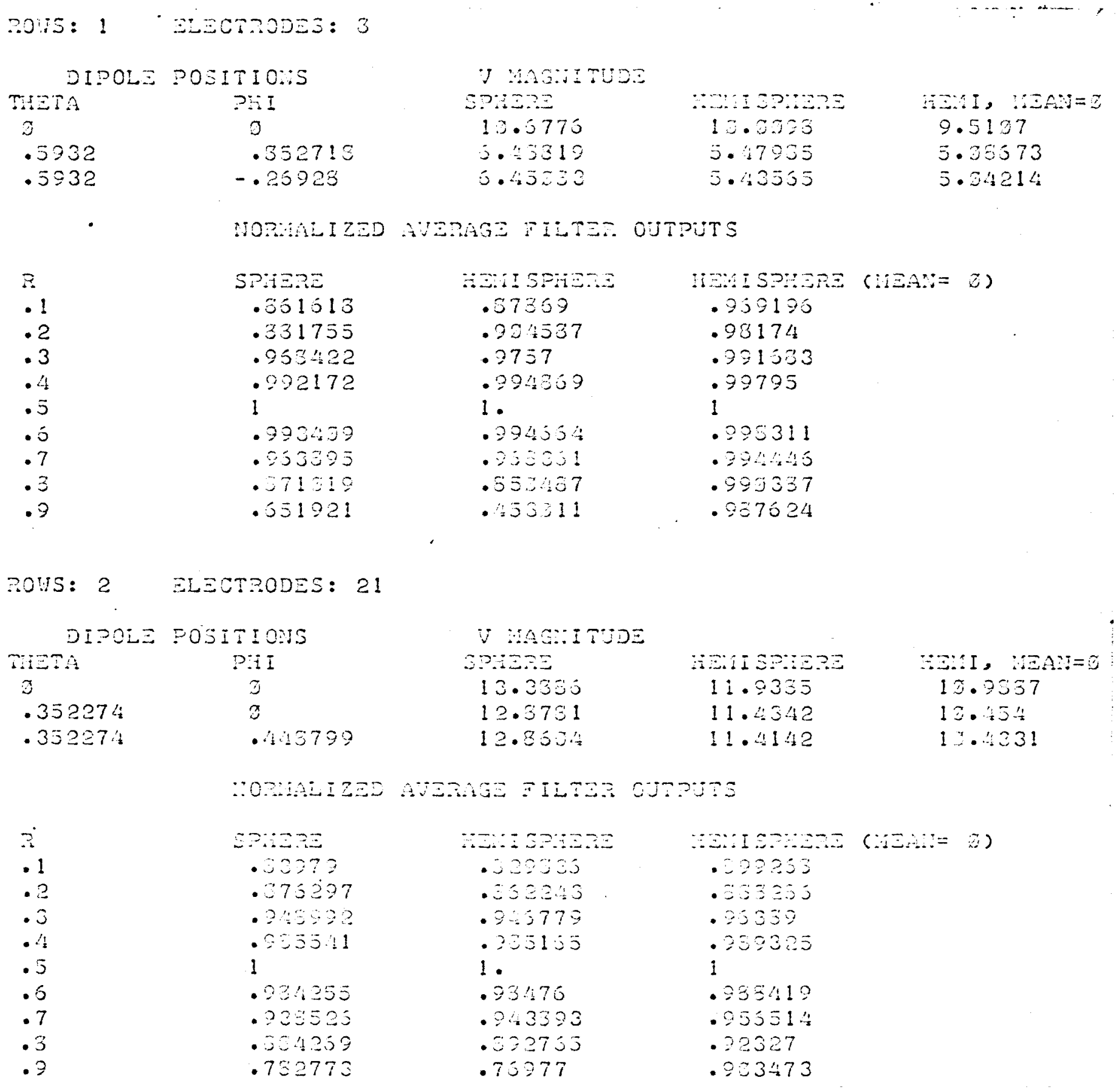

FIGURE BIf. Simuiation results for the discrete caso using a radial dipole. (Fage 1 of 3 ) 


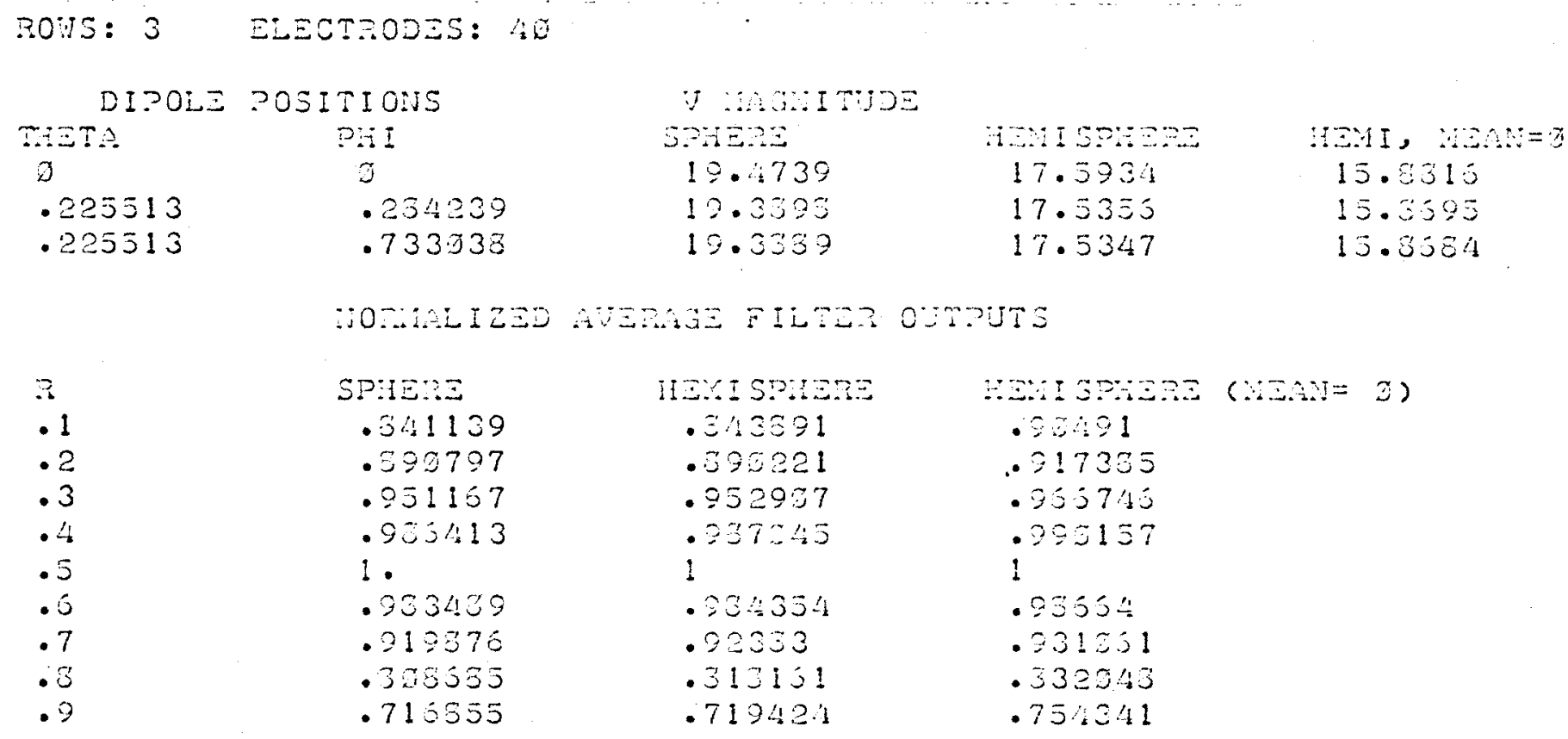

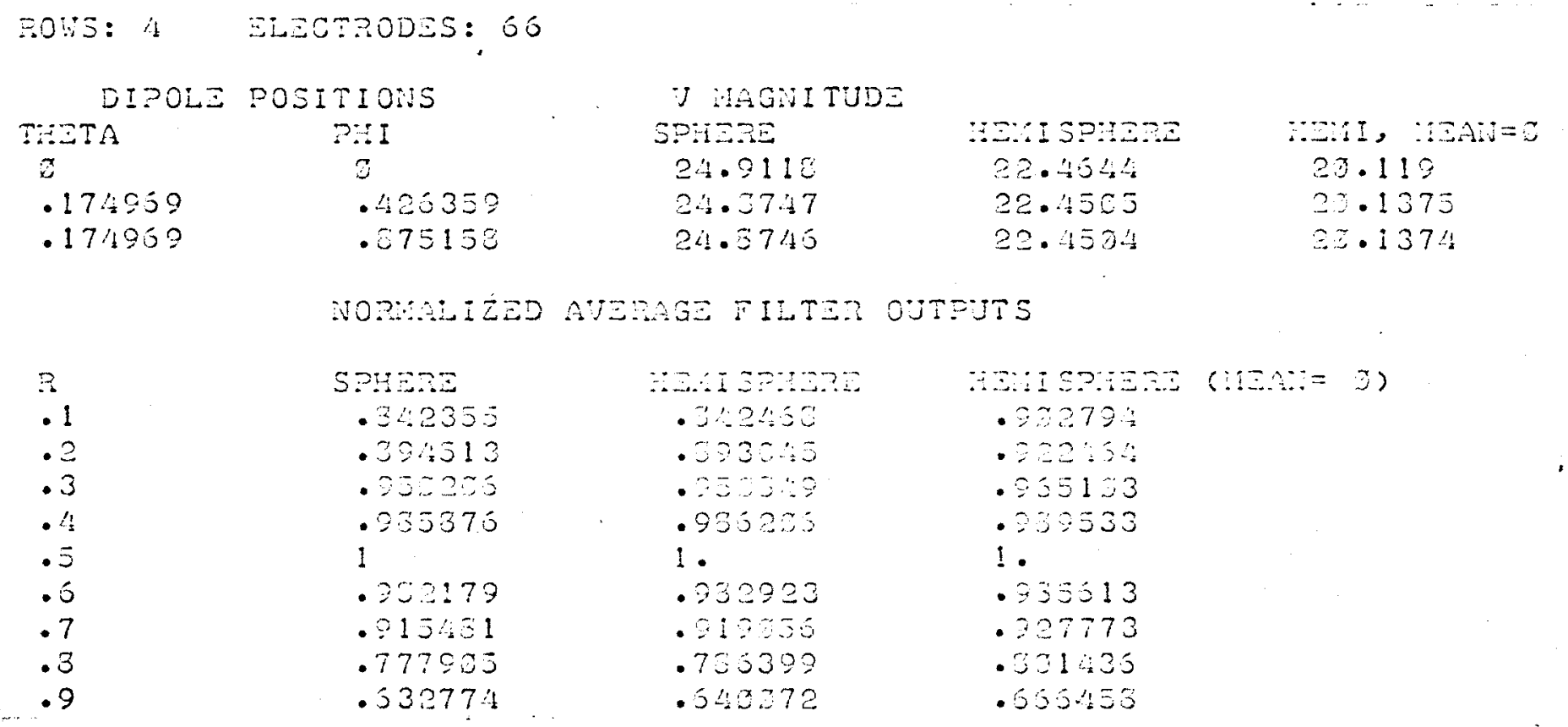

FIGURE B14. Simulation results for the discrete case lising redial dipole. (Page 2 of 3 ) 


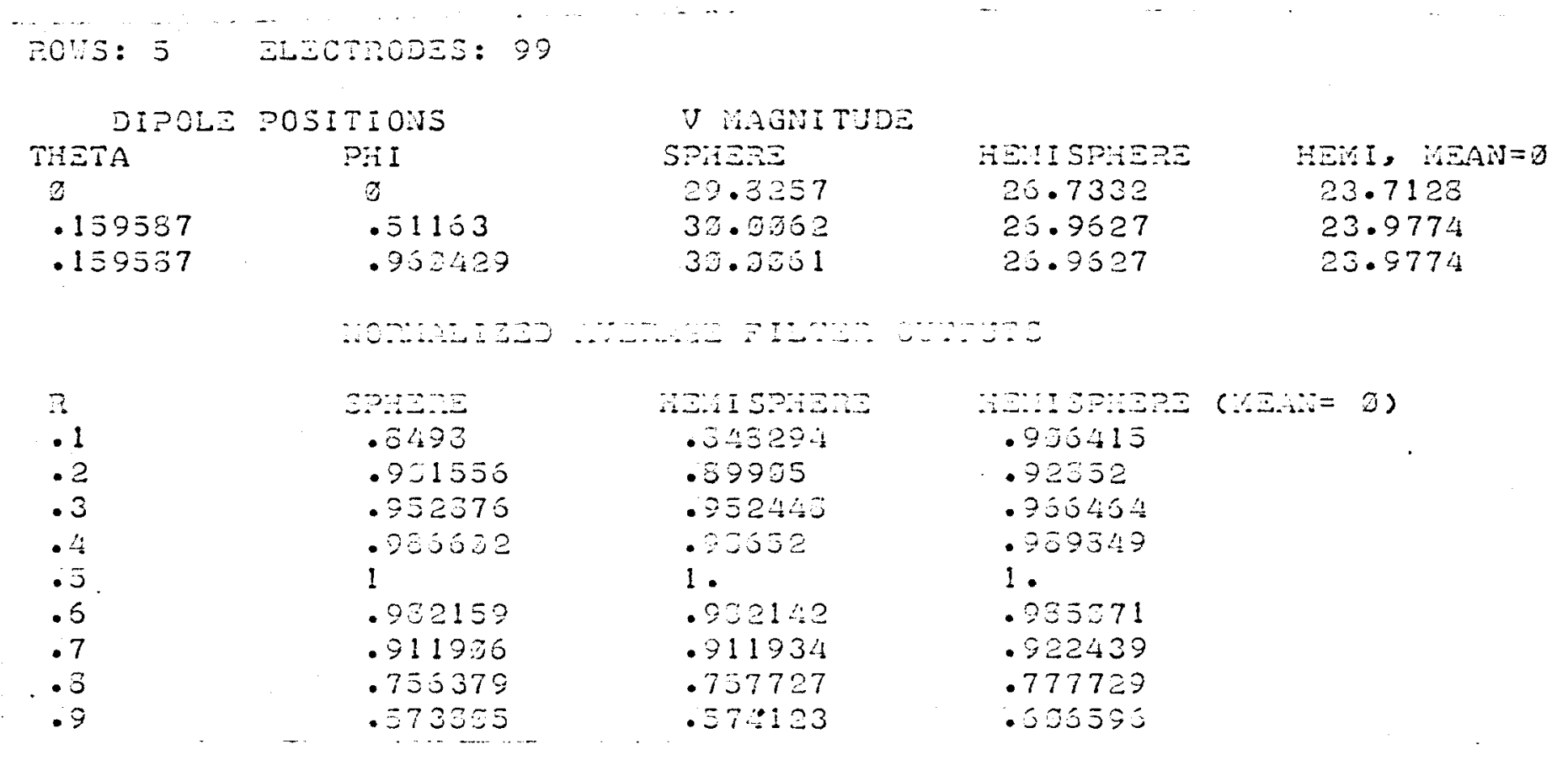

FIGURE BI4. Simulation results for the discrete case using a radial dipole. (Page 3 of 3 ) 


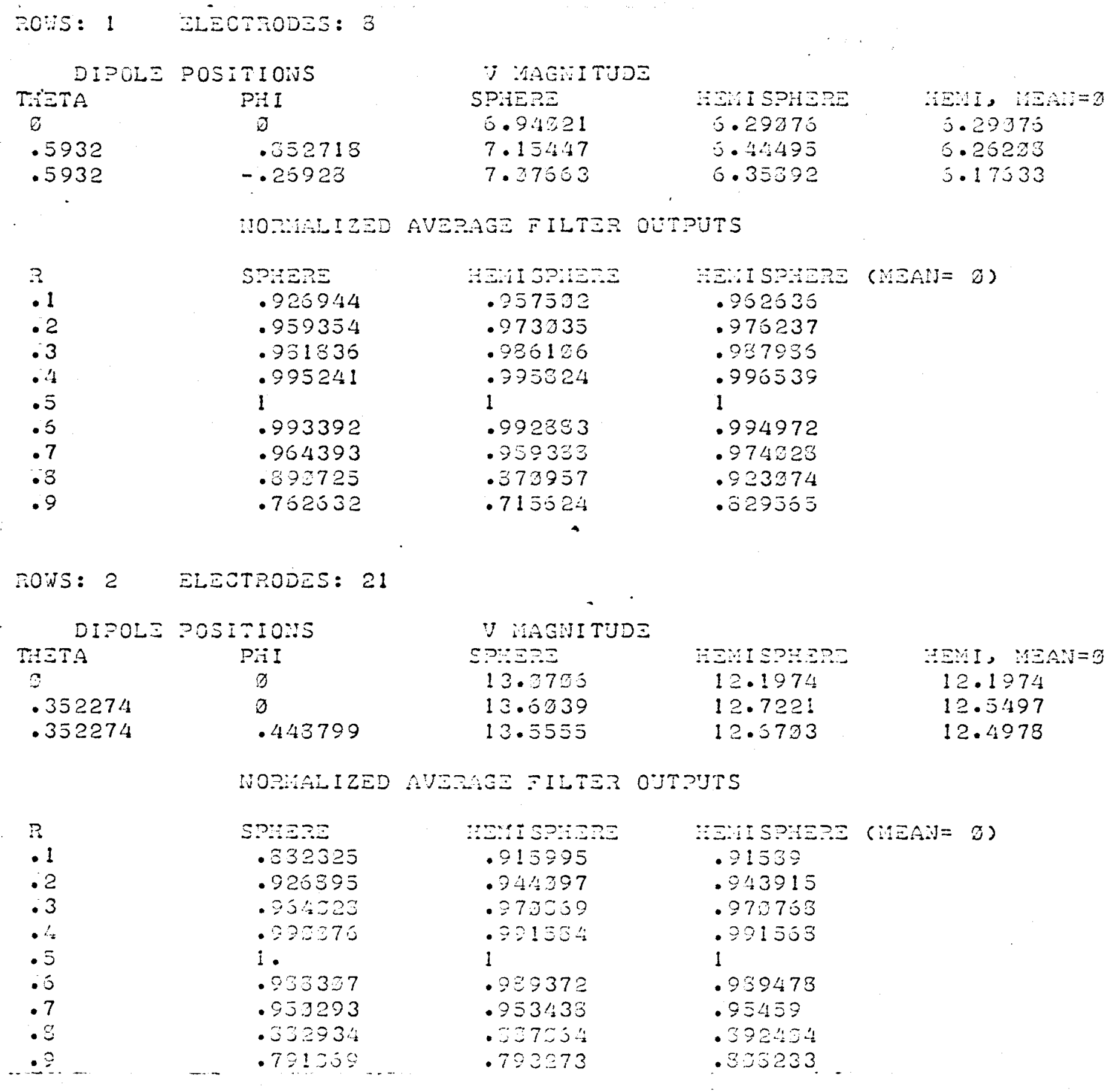

FIGURE 815. Simulation results for the discrete case using a tangential dipole. (Page 1 of 3 ) 
ROUS: 3 ELECTRODES: 43

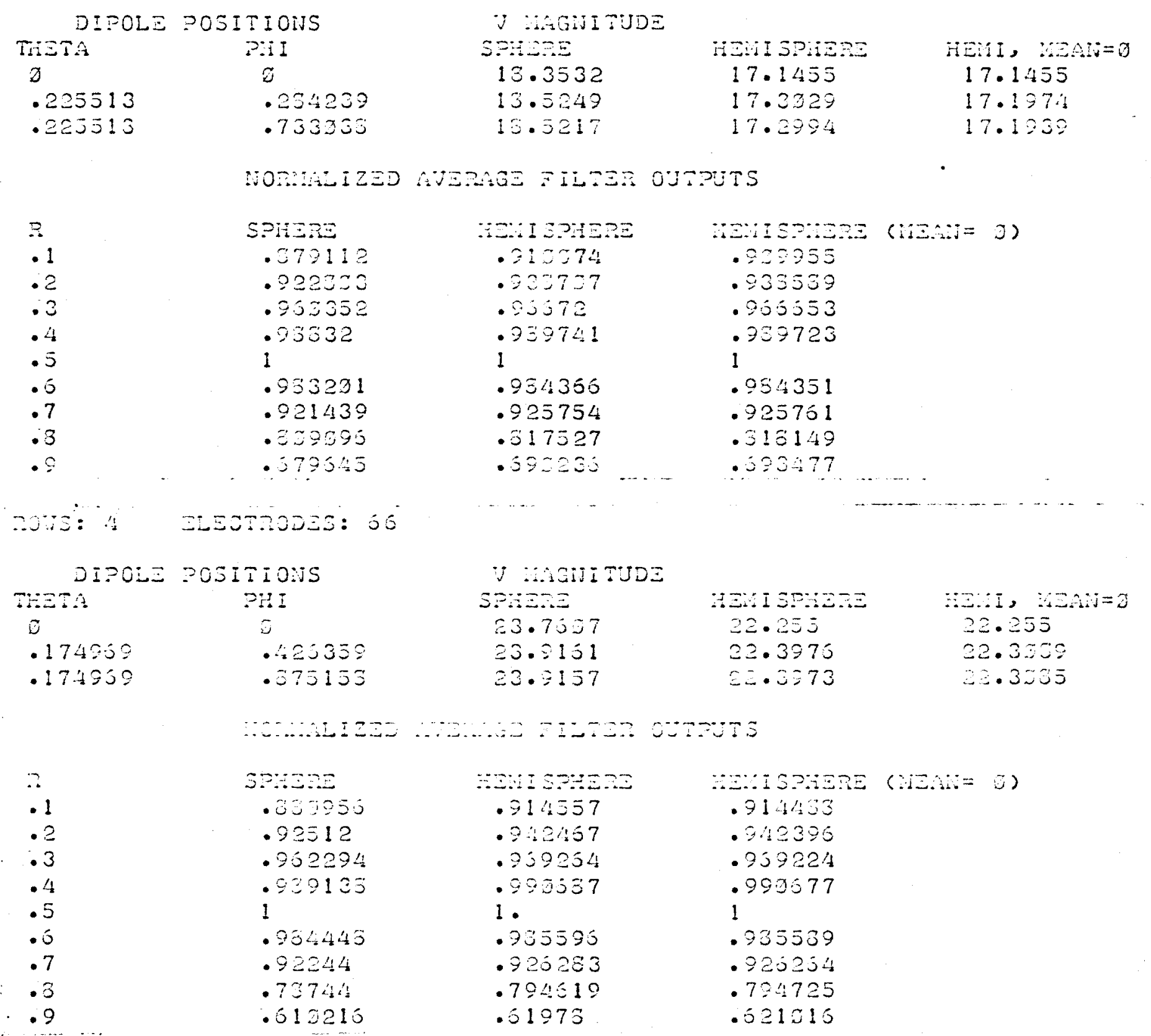

FIGURE B15. Simulation results for the discrete case using a tangential dipole. (Fage 2 of 3 ) 


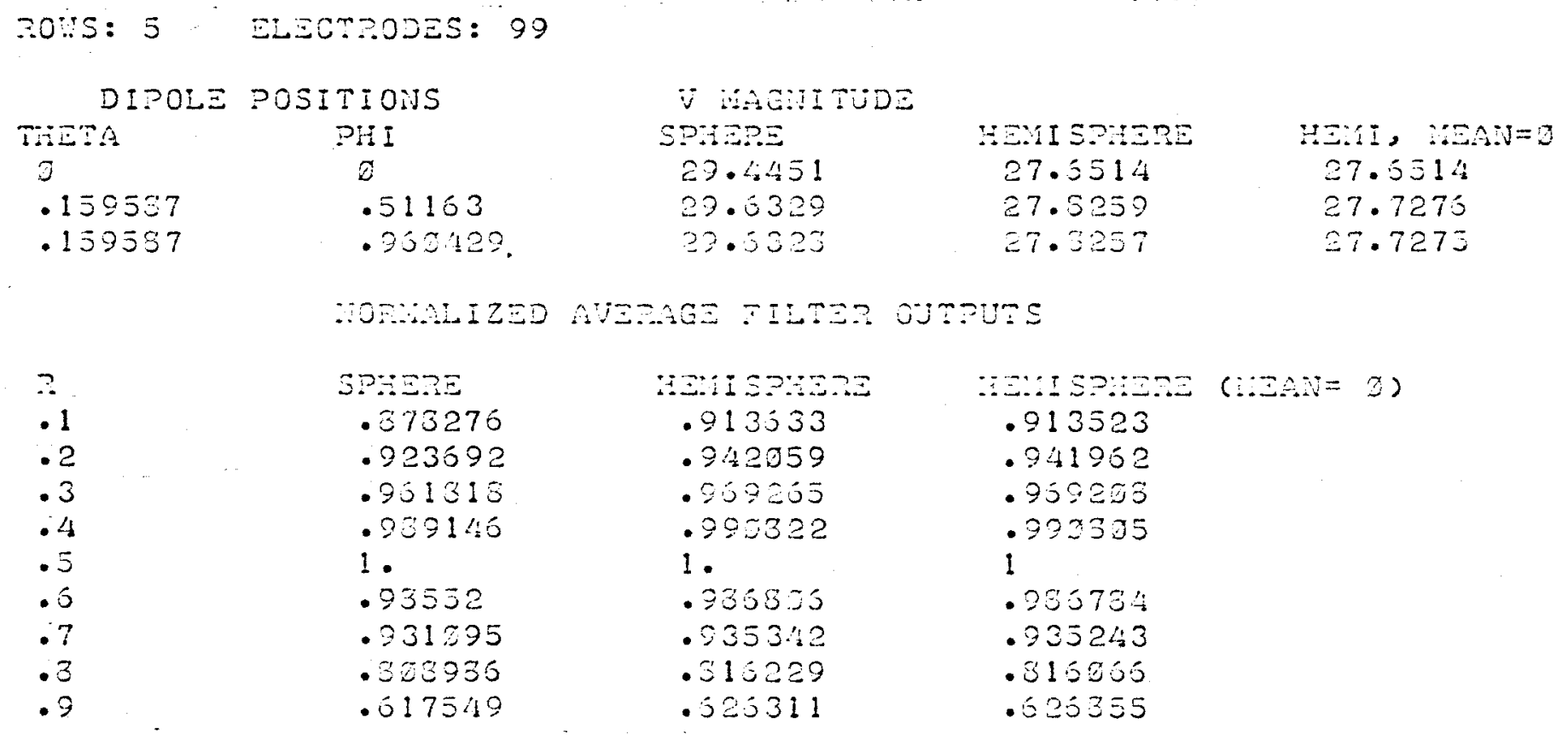


position coordinates for the surface electrodes. (2) Line 6500. This subroutine calculates the vector $V$ containing the simulated surface potentials and 1 ts magnitude.

(3) Line 7000. This subroutine calculates the vector H contalning the filter response function and its magnitude.

(4) Line 7500. This subroutins calculates the inner product of $\mathrm{V}$ and $\mathrm{H}$.

(5) Line 8000 . This subroutine performs a coordinate conversion from spherical to rectangular coordinates. (6) Line 8500. This subroutine calculates the theoretical surface potential of a current dipole. The main program and the subroutines will be described in order.

The main program performs a test for depth mismatch botween the actual dipolo and the filter dipole with varying numbers of electrodes. The actual dipole is located at a radial position of $r=0.5$. Throe positions are used, corresponding to locations directly benoath one electrode, midway betwoen two electrodos, and approximately equidistant from throe electrodes. The output printed is the average of the filter responses to the three dipoles.

The filter outputs are calculated five times, with increasing numbers of electrodes. Each time, these outputs 
are calculated for threo cases. In the first case, the electrode positions are diatributed over the entire sphere. In the second case, the electrode positions are on only one half of the sphere, namely the hemisphere for which the 2 coordinate is greater than zero. The third case is simflar to the second, with the additional constraint that the average of the surface potentials of all electrodes is zero. This third case was included to investigate the effects of alternate electrode connection schemes as discussed in chapter IV. Referring to the results shown in figures $\mathrm{Bl}_{4}$ and $B 15$, the program first prints the number of rows of electrodes and the total number of electrodes in each hemisphere. Then, the three dipole positions used ore printed, along with the calculated magnitude of the surface potential vector for each of the three cases which have been described. Then, the average filter response is printed for each case as a function of the depth of the filter dipole.

The first subroutine, which begins at line 5100 , was written to generate sets of electrode coordinates for varying numbers of electrodes. To achleve an even distribution of these electrodes over the surface, the sphere was partitioned into segments of equal area, with one electrode in eacb segment. A further constraint was that the electrodes should be approximately equidistant 
from one another. The algorithm used for electrode positioning will now be described.

The first step of the algorithm is to divide the top $(z>0)$ hemisphere into sections of equal width, which will be called bands. One band will be centered around the $z$ axis, and $n_{1}$ additional bands will be defined in this hemisphere (See fig. Bl6 for the case $\left.n_{1}=2\right)$. The total number of bands in the hemisphere is therefore $n_{1}+1$. The width of each band is then

$$
\Delta \theta=\pi /\left(2 n_{1}+1\right)
$$

It can be seen from fig. B16 that the $\theta$ coordinate of the edge of the band centered around the $z$ axis is

$$
\theta_{0}=\Delta \theta / 2
$$

B18

Note that $\theta_{0}$ is represented by variable TO in the program. The bemisphere is next divided into $n_{3}$ sections of equal area, which will be called rings (The way in which $n_{3}$ is colculated will be described later). This operation may be performed by dividing the $z$ axis from 0 to 1 into $n_{3}$ segments, each of length $\Delta z=1 / n_{3}$, as shown in fig. B17. The proof of this statement follows. Given the sphere defined by the equation $x^{2}+y^{2}+z^{2}=1$

and the segment of this sphere defined by the inequality $x^{2}+y^{2} \leq a^{2} \leq 1 ; \quad$ B2O the surface area of this segment is 27

$$
A=2 \pi\left(1-\left(1-a^{2}\right)^{\frac{1}{2}}\right) \text {. }
$$

But from fig. B17, using the Pythagorean theorem, 


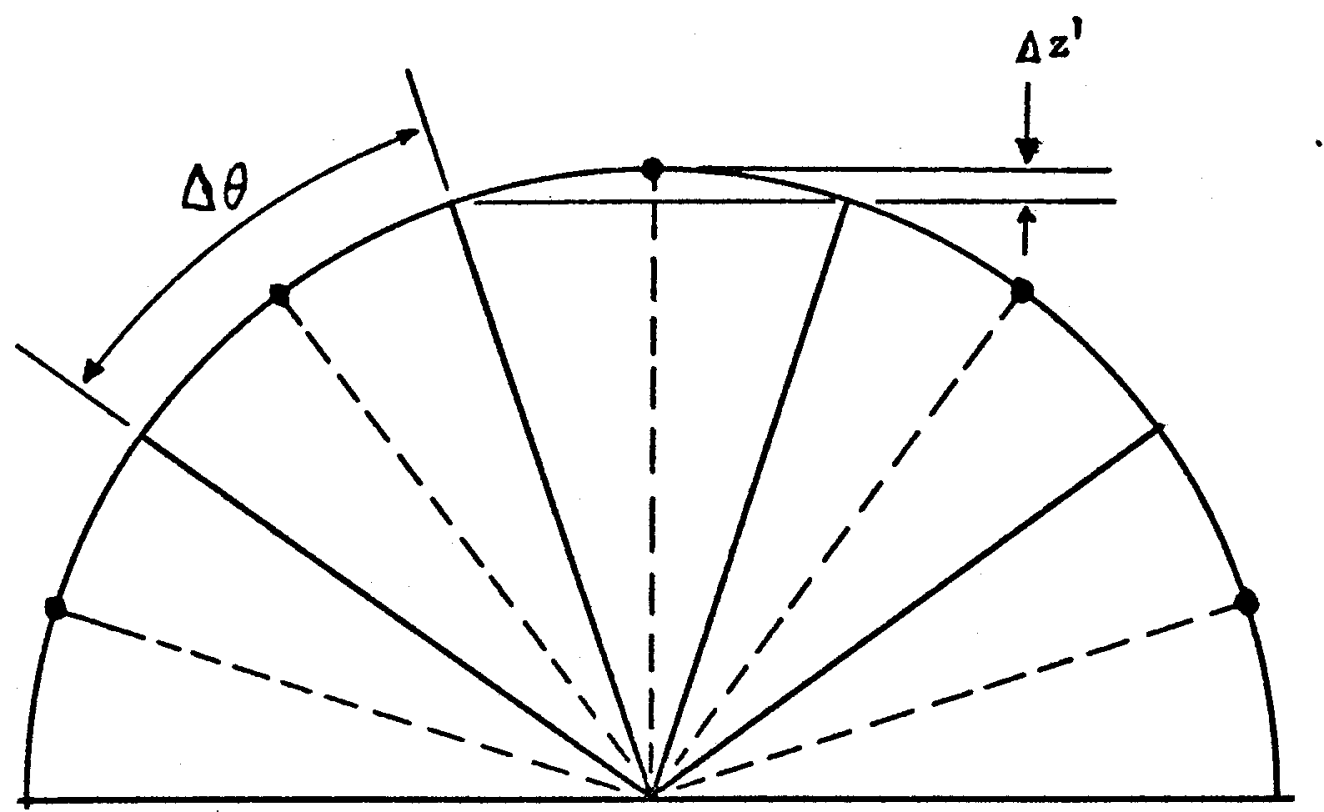

FIGURE B16. Division of a hemisphere into bands of equal width. The dots indicate the $\theta$ coordinates of the electrode positions. 


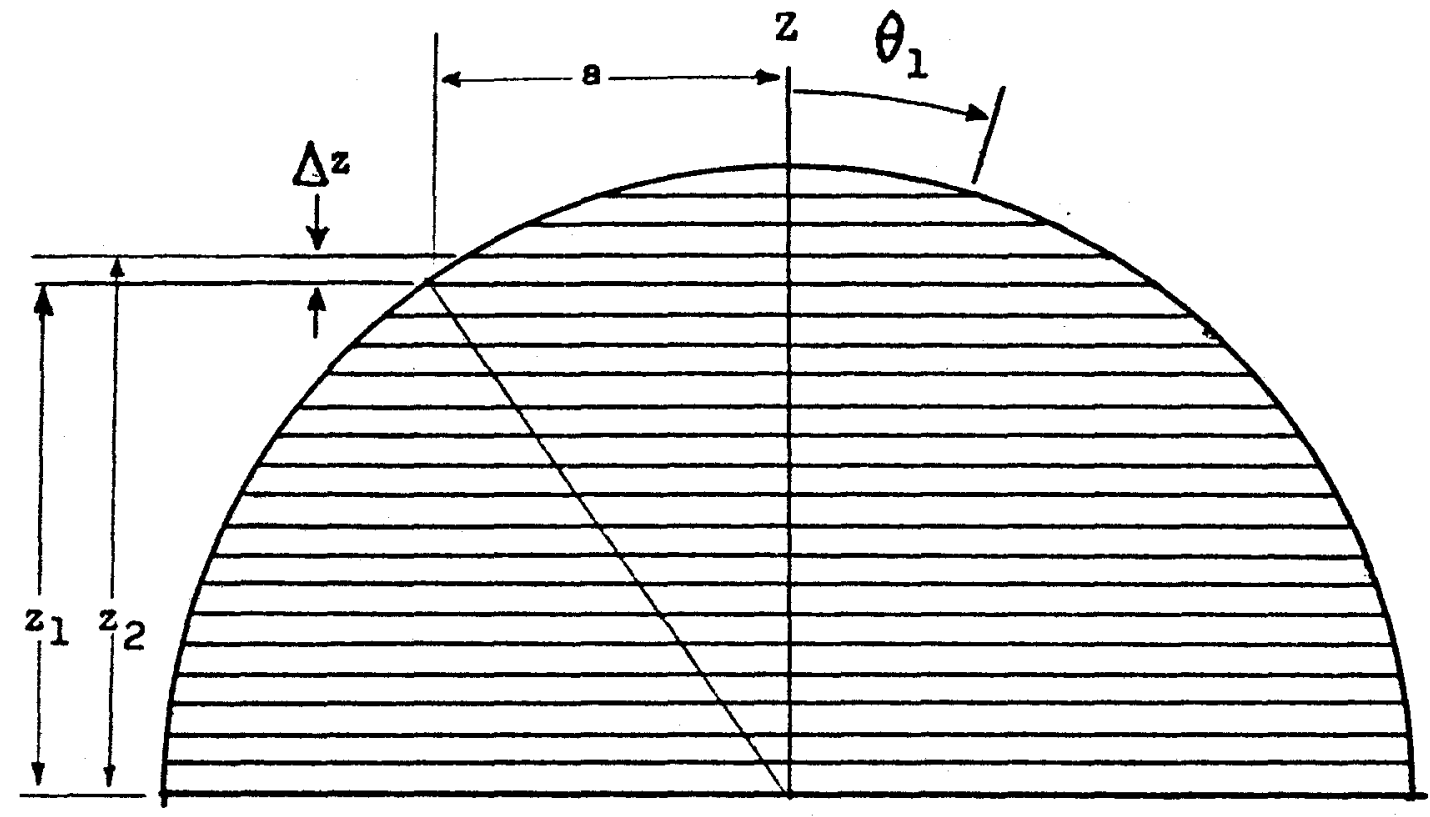

FIGURE B17. Division of a bemisphere into rings of equal area. 


$$
1-a^{2}=z_{1}^{2},
$$

or

$$
A=2 \pi\left(1-z_{1}\right),
$$

since only the upper hemisphere is being considered. Therefore, the area of the portion of the sphere between two planes parallel to the $x y$ plane and passing through points $z_{1}$ and $z_{2}$ on the $z$ axis is:

$$
\Delta A=A\left(z_{1}\right)-A\left(z_{2}\right)=2 \pi\left(z_{2}-z_{1}\right)
$$

$\mathrm{B} 24$

From this equation it can be seen that dividing the $z$ axis into equal segments of length $\Delta z$ will divide the hemisphere into rings of equal area $2 \pi \Delta z$.

In order to make the area of each ring approximately equal to the area of the top band, the number of rings, $n_{3}$, is chosen so that $\Delta z$ will be approximately equal to the distance $\Delta z^{\prime}$ shown in fig. B16. Since $\theta_{0}$ is the coordinate of the odge of the top band, this distence is

$$
\Delta z^{\prime}=1-\cos \left(\theta_{0}\right) \text {. }
$$

To meet the above constraint and also produce an integral number of divisions on the $z$ axis, $n_{3}$ is defined as

$$
n_{3}=\operatorname{INT}\left[0.999999+\frac{1}{\Delta z^{\prime}}\right]
$$

where function $\operatorname{INT}(x)$ is the greatest integer less than or equal to $x$. This value is claculated in line 5130 of the program.

After these rings and bands have been defined, the electrode positions are defined as follows. One electrode is placed at the top of the sphere; that is, at the position $r=1, \theta=0, \phi=0$. Next, electrodes 
are placed in each of the bands such that the i'th band contains $f_{1}$ electrodes, where $f_{1}$ is the number of rings in that bend. A ring which is split by the boundary between two bends is considered to be in the lower of the two bands. For the electrodes in one band, the $\theta$ position coordinate is set equal to the coordinate of the lower edge of the ring which is in the center of the band. The $\phi$ spacing between electrodes in a given band is then $2 \pi / 3_{1}$.

The loop consisting of lines 5180 through 5380 counts the number of rings in each band and stores the results in array $N$. The $\theta$ coordinates for the electrodes are stored in array $T$.

After the electrode positions are calculated in spherical coordinates, they must be converted to rectangular coordinates for use by the other subroutines. This transformation is accomplished by the portion of tho subroutine which begins at line 6100 .

The next subroutine, which begins at line 6500, calculates the vector $V$ contalning the simulated surface potentials. The magnltude of this vector is also calcu1ated. The dipole location, orientation, and megnitude 
are specified in the calling program. The electrode positions for the upper hemisphere are the coordinates calculated by the first subroutine. If variable li is zero, the vector contains volues only for points on the upper hemisphero. If Ll 1 s one, potentials are calculated for the entire sphere. In this case, the electrode coordinates for the lower homisphere are the same as for the upper hemisphere, except that $z$ is negated. After the vector is calculated, Its magnitude is evaluated by the portion of the subroutine beginning at line 6660 . This section calculates the magnitude as the square root of the sum of the squares of the vector elements.

The third subroutine, which begins at line 7000 , celculates the vector $H$ containing the filter weighting function. The operation of this subroutine is the same as that of the previous subroutine for calculating $V$. The fourth subroutine, beginning at Iine 7500, calculatos the inner product of vectors $\mathrm{V}$ and $\mathrm{H}$. The fifth subroutine, beglnning at line 8000 , performs coordinate conversions. This operation is included so that the main program can use spherical coordinates, while the potential evaluation subroutine uses rectanguler coordinates. Two conversions must be performed by this subroutine. First, the position coordinates of the dipole must be converted from spherical to rectangular coordinates. This operation is accom- 
plished by lines 8050 through 8070 . Second, the values of the $\bar{\theta}_{f}, \bar{a}_{\theta}$, ond $\bar{a}_{\phi}$ component dipoles are spectifled in the main program. The $\bar{\theta}_{x}, \bar{\theta}_{y}$, and $\bar{a}_{z}$ component dipole magnitudes are computed from these values by Ines 8080 through 8100 of this subroutine.

The sixth subroutine, beginning at line 8500 , calculateg the potential at any point on the surface given the location, orientation, and magnitude of the current dipole. This program evaluates equations A1 through $A 9$ of appendix $A$ with the radius of the sphere set equal to one and the constant $1 /(4 \pi \sigma R)$ of equation A9 also equel to one. 
APPENDIX C. EFFECTS OF NOTSE

This appendix presents a preliminary investigation of the effects of nolse on the apparent magnitude of the dipole. As stated in chapter II, it will be assumod that the noise component of the surface potential has zero mean and is white. That is, if the surface potential at point $\overline{\mathrm{r}}$ is

$$
v(\bar{x})=s(\bar{x})+n(\bar{x})
$$

where $s(\bar{r})$ is the signal component and $n(\bar{r})$ is the nolse component, then $n$ has the following properties (repeating equations II $-6 a$ and $(I-6 b)$ :

$$
\begin{aligned}
& E[n(\bar{r})]=0 \\
& E\left[n\left(\bar{r}_{1}\right) n\left(\bar{r}_{2}\right)\right]=k^{2} \delta_{S}\left(\bar{r}_{2}-\bar{r}_{1}\right)
\end{aligned}
$$

For the discrete case, these assumptions of zero mean and whiteness imply that the mean of the nolso potential at any electrode is zero, and that the cross correlation between nolse components at any two different electrodes is zero. That is, the surface potential vector $V$ is given by the equation

$$
V=S+N
$$

$\mathrm{CL}_{4}$

where $S$ is a vector of the signal potentials and $N$ is a vector containing the nolse scmponents. Now, the assumption of zero mean implies that 


$$
E(N)=0
$$

where 0 in this case implies the zero vector. Tho assumption of whiteness implies that the covariance matrix, Q, of $N$ is proportional to the identity matrix, or

$$
Q=E\left[N N^{t}\right]=k^{2} I
$$

Equation 66 is equivalent to the statement that for any two elements $n_{1}$ and $n_{g}$ of $N$,

$$
E\left[n_{1} n_{j}\right]= \begin{cases}k^{2}, & 1=j \\ 0, & 1 \neq j\end{cases}
$$

The question to be answered is bow much inaccuracy the nofse adds to the observed magnitudes of the component dipoles. The errors in the component dipole megnitudes for the continuous case w1ll be presented first; then the errors for the discrete case will be derived.

From equation II $-40 a$, the error in the $\bar{x}$ directed component of the dipole will be

$$
u_{r}=\left(\hat{d}_{r}-d_{r}\right)=\left[\int h_{\mathbf{r}}\left(\theta, \phi \mid \bar{s}_{0}\right) n(\theta, \phi) d q\right] / c_{r}\left(\bar{r}_{0}\right) \quad c 8
$$

The mean volue of the error is then

$$
E\left[u_{r}\right]=E\left[\int_{p}\left(\theta, \phi \mid \vec{r}_{0}\right) n(\theta, \phi) d q\right] / o_{p}\left(\bar{r}_{0}\right)
$$


or,

$$
E\left[u_{r}\right]=\int h_{r}\left(\theta, \phi \mid \bar{r}_{0}\right) E[n(\theta, \phi)] d q / c_{r}\left(\bar{r}_{0}\right) \quad c 10
$$

which is equal to zero since the expected velue of $n$ is zero.

Since the mean of $u_{r}$ is zero, the variance 18 simply the expected value of $u_{r}^{2}$. This parameter may be determined as follows.

First,

$$
E\left[u_{r}^{2}\right]=E\left[\int h_{r}\left(\theta, \phi \mid \bar{r}_{0}\right) n(\theta, \phi) d q\right]^{2} / 0_{r}^{2}\left(\bar{r}_{0}\right)
$$

Applying the result of oq. II-12, eq. Cll reduces to

$$
E\left[u_{r}^{2}\right]=k^{2} \int h_{r}^{2}\left(\theta, \phi \mid \bar{r}_{0}\right) d q / c_{r}^{2}\left(\bar{r}_{0}\right)
$$

However, the Integral of $h_{r}^{2}$ over the surface wes defined to be unity in eq. II-18, so that the variance of $u_{p}$

is then

$$
E\left[u_{r}^{2}\right]=k^{2} / c_{r}^{2}\left(\bar{r}_{0}\right)
$$

A similar development may be applied to the other two component dipoles, so that the mean values of the nolso induced orror in the component magnitudes are

$$
\begin{array}{ll}
E\left(u_{p}\right)=0 & c 14 a \\
E\left(u_{\theta}\right)=0 & c 14 b \\
E\left(u_{\phi}\right)=0 & c 14 c
\end{array}
$$


The vartances of these errors are

$$
\begin{aligned}
& E\left(u_{r}^{2}\right)=k^{2} / c_{r}^{2}\left(\bar{r}_{0}\right) \\
& E\left(u_{\theta}^{2}\right)=k^{2} / c_{\theta}^{2}\left(\bar{r}_{0}\right) \\
& E\left(u_{\phi}^{2}\right)=k^{2} / c_{\phi}^{2}\left(\bar{r}_{0}\right)
\end{aligned}
$$

In summary, it can be seen from eq. Cll that the mean values of the component dipole magnitudes as calculated using the matched filter technique will be equal to the actual values. In addition, the variances of the orrors in the calculated magnitudes will be a function of the input nolso variance as given in eq. CI5.

EFFECTS ON MAGNITUDE FOR THE DISCRETE CASE

From eq. II $-60 a$, the error in the magnitude of the $\bar{E}_{r}$ directed component of the dipole will be

$$
u_{r}=\left(\hat{d}_{r}-d_{r}\right)=H_{r}^{t}\left(\bar{r}_{0}\right) N / c_{r}\left(\bar{r}_{0}\right)
$$

The mean value of $u_{p}$ is then

$$
E\left(u_{r}\right)=E\left[H_{r}^{t}\left(\bar{r}_{0}\right) N\right] / c_{r}\left(\bar{r}_{0}\right)
$$

or

$$
E\left(u_{r}\right)=H_{r}^{t}\left(\bar{r}_{0}\right) E[N] / c_{r}\left(\bar{r}_{0}\right)
$$

which is zero since the expected value of $\mathrm{N}$ is the 
zero vector, from eq. C5.

Since the mean of $u_{r}$ is zero, the variance is simply the expected value of $u_{x}^{2}$;

$$
E\left(u_{r}^{2}\right)=E\left[\left(H_{r}^{t}\left(\bar{r}_{0}\right) N\right)\left(H_{r}^{t}\left(\bar{r}_{0}\right) N\right)\right] / c_{r}^{2}\left(\bar{r}_{0}\right)
$$

or,

$$
E\left(u_{r}^{2}\right)=E\left[H{ }_{r}^{t}\left(\bar{r}_{0}\right) W N t_{r}\left(\bar{r}_{0}\right)\right] / c_{r}^{2}\left(\bar{r}_{0}\right)
$$

or,

$$
E\left(u_{r}^{2}\right)=H_{r}^{t}\left(\bar{r}_{0}\right) E[N N]_{H_{r}}\left(\bar{r}_{0}\right) / c_{r}^{2}\left(\bar{r}_{0}\right)
$$

which, by eq. C6, reduces to

$$
E\left(u_{r}^{2}\right)=k^{2} \mathrm{H}_{r}^{t}\left(\bar{r}_{0}\right) \mathrm{H}_{r}\left(\bar{r}_{0}\right) / c_{r}^{2}\left(\bar{r}_{0}\right)
$$

Now, by eq. II-39, this equation becomes

$$
E\left(u_{r}^{2}\right)=k^{2} / c_{r}^{2}\left(\bar{r}_{0}\right)
$$

By repeating this derivation for the other two components, it is found that the average error for each component is

$$
\begin{aligned}
& E\left(u_{p}\right)=0 \\
& E\left(u_{\theta}\right)=0 \\
& E\left(u_{\phi}\right)=0
\end{aligned}
$$

The variances of these nolse components are

$$
\begin{aligned}
& E\left(u_{r}^{2}\right)=k^{2} / c_{r}^{2}\left(\bar{r}_{0}\right) \\
& E\left(u_{\theta}^{2}\right)=k^{2} / c_{\theta}^{2}\left(\bar{r}_{0}\right) \\
& E\left(u_{\phi}^{2}\right)=k^{2} / c_{\phi}^{2}\left(\bar{r}_{0}\right)
\end{aligned}
$$

Therefore, for the discrete case as well as the con- 
tinuous case, the average value of the error in the dipole component magnitudes is zero. In addition, the output nolse variance is again proportional to the input varlance, as shown in eq. C25. 


\section{BIBLIOGRAPHY}

\section{Sources Cited}

1. R. Plonsey, Bloelectric Phenomena. Now York, McGrawH111, 1969, pp. 202-275.

2. Ibid., pp. 324-332.

3. Ibld., pp. 202-209.

4. S. Rush and D. A. Driscoll, "EEG Electrode Sensitivity An Application of Reciprocity," IEEE Trans. Blomed. Eng., vol. BME-16, pp. 15-22, Jan. 1969.

5. S. Rush and D. A. Driscoll, "Current Distribution in the Brain from Surfece Electrodes," Anesthegia and Analgesia, vol. 47, pp. 717-723, Nov.-Dec. 1968.

6. M. R. Scbnelder, "A Multistage Process for Computing Virtual Dipolar Sources of EEG Discharges from Surface Information," IEEE Trans. Bloned. Eng., 701. BME-19, pp. 1-12, Jan. 1972.

7. L. J. PInson and D. G. Chllders, Frequency-Wavenumber Spectrum Analysis of EEG Multielectrode Array Data," IEEE Trans. Biomed. Eng., vol. BME-21, pp. 192-206, May 1974 .

8. S. Servit, A. Strejekova, and D. Volanschl, "An Epileptogenic Focus in the Frog Telencephalon. Pathways of Propagation of Focal Activity," Exp. Neurol., vol. 21, pp. 383-396, 1968.

9. P. Morroll, "Secondary Eplleptogenic Lesions," Epliopsia, vol. 1, pp. 538-560, 1960.

10. R. L. Isascson, H. Schwartz, N, Porsoff, and L. Pinson, "The Role of the Corpus Collosum in the EstabIishrent of Areas of Socondary Epileptiform Activity," Epilepsia, vol. 12, pp. 133-146, 1971.

11. C. L. Rogers and T. C. Plikington, "Froe-koment Current Dipoles in Inverse Electrocerdiography," IEEE Trans. Blomed. Eng., vol. BME-15, pp. 312-323, oct. 1968. 
12. C. L. Rogers and T. C. Pilkington, "The Solution of Overdetermined Linear Equations as a Multistage Process," IEEE Trans. Blomod. Eng., vol. BME-15, pp. 179-185, July 1968.

13. L. A. Zedob and C. A. Desoer, Linear System Theory The State Spoce Approach. New York, McGraw-Hil1, 1963, pp. 577-582.

14. W. H. Plerce, Statistlcally Perlodic Communications. Unpublished Manuscript.

15. R. C. Barr and T. C. Plikington, "Computing Inverse Solutions for an on-off Heart Model," IEEE Trans. Blomed. Eng., vol. BME-16, pp. 205-214, JuIg 1969.

16. R. C. Barr, T. C. Plikington, J. P. Boineau, and C. L. Rogers, "Án Inverse Electrocardiograpble Solution with an on-off Hodol," IEEE Trans. Blomed. Eng., vol. BME-17, pp. 49-57, Jan. 1970.

17. M. Lynn, A. Barnard, and J. Holt, "A Proposed Method for the Inverse Problem in Electrocardlography," Blophys. J.. vol. 7, pp. 925-945, 1967.

18. F. N. Wilson and R. H. Bajley, "The Electric Field of an Eccentric Dipole in a Homogeneous Spherical Conducting Modium," Circulation, vol. 1, pp. 84-92, 1950.

19. M. Schneider, "Effect of Inhomogeneities on Surface Stgnals Coming from a Corobral Current-Dipolo Source," IEEE Trans. Biomed. Eng., vol. BME-21, pp. 52-54, Jan. 1974.

20. M. Schwartz, Information, Transmission, Modulation, and No1se. Now York, HcGraw-I111, 1970, pp. 410-416.

21. P. M. Morse and H. Feshbech, Methods of Theoretical PhIs1cs. New York, McGraw-H111, 1953, pp. 825-830.

22. A. Papoulis, Probabil1ty, Random Varlables, and Stochastic Processes. Now York, McGraw-ilII, I965, pp. 323,324 .

23. G. Mostow and J. Sampson, Linear Algebra. New York, McGraw-Hill, 1969, pp. 22,44.

24. P. Strong, Biophys1cal Measurements. Beaverton, Oro., Tektronix, 1970, pp. 138-140. 
25. P. Stark, Introduction to Numerical Mothods. Now York, Macm1lian, 1970, pp. 212-219.

26. E. Fadell and A. Fadoll, Calculus. Now York, Van Nostrand Roinbold, 1970, pp. 542-544.

27. Ib1d., pp. 661 . 


\section{VITA}

Joseph Harvey Rothweiler was born in Louisville, Kentucky on November 30,1952 . He received the B.S. degree from the University of Loulsville Speed Sclentific School in May, 1974. From June, 1974 to the present, Mr. Rothweller has been employed by RCA Corporetion in Somerville, New Jersey. While at RCA, he has engaged in the design of digital slgnel processing hardware and in the development of charge coupled devices for signal processing applications.

Mr. Rothweiler is a member of the Institute of Electrical and Electronics Engineers, is an associate member of Sigma X1, and is cosuthor of a paper in the field of digital signsl processing. 